\title{
The exoticness and realisability of twisted Haagerup-Izumi modular data
}

\author{
DAVID E. EVANS \\ School of Mathematics, Cardiff University, \\ Senghennydd Road, Cardiff CF24 4AG, Wales, U.K. \\ e-mail: EvansDE@cf.ac.uk \\ Terry Gannon \\ Department of Mathematics, University of Alberta, \\ Edmonton, Alberta, Canada T6G 2G1 \\ e-mail: tgannon@math.ualberta.ca
}

October 29, 2018

\begin{abstract}
The quantum double of the Haagerup subfactor, the first irreducible finite depth subfactor with index above 4 , is the most obvious candidate for exotic modular data. We show that its modular data $\mathcal{D} H g$ fits into a family $\mathcal{D}^{\omega} \mathrm{Hg}_{2 n+1}$, where $n \geq 0$ and $\omega \in \mathbb{Z}_{2 n+1}$. We show $\mathcal{D}^{0} \mathrm{Hg}_{2 n+1}$ is related to the subfactors Izumi hypothetically associates to the cyclic groups $\mathbb{Z}_{2 n+1}$. Their modular data comes equipped with canonical and dual canonical modular invariants; we compute the corresponding alpha-inductions etc. In addition, we show there are (respectively) 1, 2, 0 subfactors of Izumi type $\mathbb{Z}_{7}, \mathbb{Z}_{9}$ and $\mathbb{Z}_{3}^{2}$, and find numerical evidence for 2 , 1, 1, 1, 2 subfactors of Izumi type $\mathbb{Z}_{11}, \mathbb{Z}_{13}, \mathbb{Z}_{15}, \mathbb{Z}_{17}, \mathbb{Z}_{19}$ (previously, Izumi had shown uniqueness for $\mathbb{Z}_{3}$ and $\mathbb{Z}_{5}$ ), and we identify their modular data. We explain how $\mathcal{D H g}$ (more generally $\mathcal{D}^{\omega} \mathrm{Hg}_{2 n+1}$ ) is a graft of the quantum double $\mathcal{D} \operatorname{Sym}(3)$ (resp. the twisted double $\mathcal{D}^{\omega} D_{2 n+1}$ ) by affine so(13) (resp. so $\left(4 n^{2}+4 n+5\right)$ ) at level 2. We discuss the vertex operator algebra (or conformal field theory) realisation of the modular data $\mathcal{D}^{\omega} \mathrm{Hg}_{2 n+1}$. For example we show there are exactly 2 possible character vectors (giving graded dimensions of all modules) for the Haagerup VOA at central charge $c=8$. It seems unlikely that any of this twisted Haagerup-Izumi modular data can be regarded as exotic, in any reasonable sense.
\end{abstract}

\section{Contents}

1 Introduction 
1.1 Modular data and modular invariants . . . . . . . . . . . . . 6

1.2 Subfactors, quantum doubles and tube algebras . . . . . . . . . . . . . 8

1.3 The canonical and dual canonical modular invariants . . . . . . . . . . . . . 11

1.4 VOAs and vector-valued modular functions . . . . . . . . . . . . . . . 13

2 Comparing the Haagerup, Sym(3) and $\mathrm{SO}(13) \quad \mathbf{1 6}$

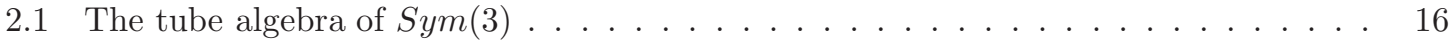

2.2 The Haagerup tube algebra $\ldots \ldots \ldots \ldots \ldots \ldots \ldots \ldots \ldots$

2.3 Haagerup modular data $\mathcal{D} H g \ldots \ldots \ldots \ldots$

2.4 Clarifying the Haagerup-Sym $(3)$ relation . . . . . . . . . . . . . . . . . . 20

2.5 The Haagerup and $\mathrm{SO}(13) \ldots \ldots \ldots \ldots \ldots \ldots \ldots \ldots$

3 Generalising the modular data of the Haagerup 24

3.1 Dihedral groups and orthogonal algebras . . . . . . . . . . . . . . . 24

3.2 Generalising the Haagerup modular data . . . . . . . . . . . . . . . . . . 25

3.3 Further generalisations . . . . . . . . . . . . . . . . . . . 27

3.4 Miscellanea involving modular invariants . . . . . . . . . . . . . . . . 30

4 Subfactors for Haagerup-Izumi modular data 33

4.1 Izumi's subfactors and their modular data . . . . . . . . . . . . . . . . 33

4.2 Principal graphs, $\alpha$-induction etc for Izumi's subfactors . . . . . . . . . . . . . . 40

5 VOAs for Haagerup-Izumi modular data 44

5.1 The Haagerup-dihedral diamond . . . . . . . . . . . . . . . . . . 44

5.2 Character vectors for the untwisted Haagerup double . . . . . . . . . . . . . 45

5.2.1 Untwisted Haagerup at central charge $c \equiv_{24} 16 \ldots \ldots \ldots \ldots$. . . . . . 45

5.2.2 Untwisted Haagerup at central charge $c \equiv_{24} 0 \ldots \ldots$. . . . . . . . . . . . 47

5.3 Character vectors of the twisted Haagerup double . . . . . . . . . . . . . . . . 47

5.3.1 The 1-twisted Haagerup at central charge $c \equiv_{24} 8 \ldots \ldots \ldots \ldots$. . . . . 48

5.3.2 The 1-twisted Haagerup at central charge $c \equiv_{24} 16 \ldots \ldots \ldots$. . . . . . 48

5.3.3 The 1-twisted Haagerup at central charge $c \equiv_{24} 0 \ldots \ldots \ldots \ldots$. . . . . . 49

5.3.4 The 2-twisted Haagerup at central charge $c \equiv_{24} 8 \ldots \ldots \ldots \ldots$. . . . . 49

5.3.5 The 2-twisted Haagerup at central charge $c \equiv_{24} 16 \ldots \ldots \ldots \ldots$

5.3.6 The 2-twisted Haagerup at central charge $c \equiv_{24} 0 \ldots \ldots \ldots \ldots$

References 50

\section{Introduction}

From the early years of conformal field theory (CFT) (see e.g. [51, 60]), we find:

Speculation. The standard constructions (orbifolds, cosets, simple-current extensions, ...) applied to the basic theories (lattice compactifications, affine Kac-Moody algebras, ...) exhaust all rational theories.

Perhaps this should be applied to the modular tensor category rather than the full CFT, in which case this would constitute a sort of generalised Tannaka-Krein duality holding for modular tensor categories, where the dual to the category (i.e. the analogue of the compact group) is (say) a vertex operator algebra constructed in a standard way from the basic examples. 
Possible counterexamples have been around just as long. For instance Walton [59] explained that rank-level duality applied to conformal embeddings of affine algebra CFTs (the simplest being $A_{1,10} \subset C_{2,1}$, yielding an extension of $A_{9,2}$ ) yield what seem to be new CFTs. The possibility that rank-level duality is somehow inherently sick was eliminated by $\mathrm{Xu}$ [61, who realised many of these examples with completely rational nets of subfactors (these should correspond to rational CFT). Perhaps all that this accomplishes is to insist that rank-level duality (or in subfactor language the mirror) should be included as one of those standard constructions. More recently, [19] proposed four modular data (each with 6 or 7 primaries) as possible counterexamples to this speculation, but didn't show they could be realised by a rational CFT (or vertex operator algebra or net of subfactors).

Perhaps the most obvious place to look for a truly exotic example is the Haagerup subfactor [1]. It is the first irreducible finite depth subfactor with index greater than 4 - it has index $(5+\sqrt{13}) / 2 \approx 4.30278$. It is generally regarded as exotic, since (so far) it can only be constructed by hand without any natural algebraic symmetries. However it is nonbraided and so to get a braided system (which has a chance to correspond to the fusion ring and modular data of a rational CFT) we should take its quantum double, or equivalently asymptotic inclusion (see Ocneanu as in [25, Chapter 12]) or Longo-Rehren inclusion [49, 50]. Its modular data $\mathcal{D} H g$ was computed in [38], and subfactor realisations of its modular invariants were studied in [26].

Question 1. Is this Haagerup quantum double realized by a rational CFT (or rational vertex operator algebra - see Definition 3 below - or completely rational net of subfactors 457 ), in the sense that they share the same modular data $\mathcal{D H g}$ ?

We would want a deeper relation between the Haagerup double and the corresponding vertex operator algebra (VOA) than merely that their modular data coincide, but for now that can suffice.

A more direct realisation of the Haagerup subfactor $N \subset M$ in a rational CFT could be that its $N-N$ sectors (say) form the algebra of defect lines (or full system) of a rational CFT. After all this will in general fail to be commutative. However, even this cannot happen: the modular data of the quantum double of a full system must be in factorised form $\mathcal{X} \otimes \mathcal{X}^{\text {opp }}$. This is because the quantum double of the full system is equivalent to the double of the fusion algebra $\mathcal{X}$ by [52, [53, Cor. 2.2], and this double factors as $\mathcal{X} \otimes \mathcal{X}^{\text {opp }}$ by [24, Prop. 2.2] as long as the braiding on $\mathcal{X}$ is non-degenerate. However, the modular data of the double of the Haagerup is easily seen to not be in factorised form [35. There remains the possibility, which we won't explore in this paper, that the Haagerup systems are the full system of a degenerately braided system. Note that much of the theory of modular invariants, including alpha-induction and the full system, holds for degenerately braided systems [11, 12, 8, 9, 10].

Question 2. If $\mathcal{D H g}$ is realised by a rational VOA, say, then is that VOA exotic, in the sense that it cannot be constructed from standard methods and examples?

The three known constructions of the Haagerup subfactor (namely Haagerup's 
connection computation [34, 1], Izumi's Cuntz algebra construction [38] and Peters' planar algebra construction [54]) are largely combinatorial tours de force (although Izumi's suggests an underlying cyclic group), and for this reason the Haagerup subfactor is generally regarded as exotic. Even given this, it is conceivable that its quantum double $\mathcal{D H g}$ could be constructed directly in more standard ways. [35] confirm that $\mathcal{D} H g$ does not fall into the simplest possibilities, namely the modular data of an affine algebra, lattice, or finite group.

We argue that the answer to Question 1 is yes, and that for Question 2 is no.

In particular, we explain why such a VOA should be a conformal subalgebra of the central charge $c=8 \mathrm{VOA} \mathcal{V}\left(E_{6} A_{2}\right)$ corresponding to (root) lattice $E_{6} \oplus A_{2}$ (by conformal subalgebra we mean a subVOA of identical central charge and identical conformal vector). A more familiar conformal subVOA of $\mathcal{V}\left(E_{6} A_{2}\right)$ is an order-2 orbifold, realising the modular data $\mathcal{D} S_{3}$ of the quantum double of the symmetric group $\operatorname{Sym}(3)=S_{3}$. An early indication for us that the Haagerup is related to $S_{3}$ was the similarity of their tube algebras. Tube algebras were introduced by Ocneanu (see e.g. [25]) as a way of computing the irreducible objects and fusion rules of the quantum doubles of subfactors, and developed further by Izumi [37, 38, in particular in analysing and determining their modular data. The list of modular invariants and nimreps for the doubles of the Haagerup and $S_{3}[15,26$, 27] are also strikingly similar, and in fact the modular invariants lead us to $E_{6} \oplus A_{2}$. The connection between modular data and VOAs is made much more explicit using character vectors. A second, equally promising possibility for a VOA realisation of $\mathcal{D H g}$, by GKO cosets using the affine algebra $\operatorname{VOA} \mathcal{V}\left(B_{6,2}\right)$, is discussed but not analysed in detail.

We generalize the modular data $\mathcal{D H g}$ in two directions, by fiting it into an infinite sequence, and showing the $n$th term in this sequence can be twisted by $\mathbb{Z}_{2 n+1}$. The role of $S_{3}$ is now played by the twisted quantum double of $D_{2 n+1}$, and $\mathcal{V}\left(E_{6} A_{2}\right)$ (which realises the $\mathcal{D} \mathbb{Z}_{3}$ modular data) by a holomorphic orbifold by $\mathbb{Z}_{2 n+1}$. Subfactor realizations of $\mathcal{D}^{0} \mathrm{Hg}_{2 n+1}$ should be provided by Izumi's hypothetical generalisation of the Haagerup: we construct a unique subfactor of Izumi type which realises $\mathcal{D}^{0} \mathrm{Hg}_{\nu}$ for all $1 \leq \nu \leq 19$ and conjecture that indeed this continues for every $n$. For this reason we suggest calling $\mathcal{D}^{0} \mathrm{Hg}_{2 n+1}$ Haagerup-Izumi modular data. von Neumann algebra realisations of twisted Haagerup-Izumi modular data $\mathcal{D}^{\omega} \mathrm{Hg}_{2 n+1}$ are still unclear.

Question 3. Can we orbifold a VOA by something more general than a group?

If so, this should provide a simple construction of VOAs realising $\mathcal{D}^{\omega} \mathrm{Hg}_{2 n+1}$, starting from a holomorphic orbifold by $\mathbb{Z}_{2 n+1}$. Perhaps this is not that unreasonable - after all, a subfactor itself is really a generalised orbifold, in the sense that the quantum double of a subfactor $M^{G} \subset M$ of fixed points recovers $\mathcal{D} G$. Indeed the whole motivation of subfactor theory (see e.g. [25]) is to understand non-grouplike quantum symmetries through subfactors or inclusions of factors $N \subset M$, and associated notions of $N-M, N-M, M-M$ irreducible bimodules or sectors, their fusion rules, paragroups, $\lambda$-lattices or planar algebras. This notion of generalised orbifold construction also features in the framework of [30].

Our work suggests a further generalisation. Consider a quadruple $(K, H, \alpha, \omega)$ 
where $K$ and $H$ are finite groups, and $H$ acts freely on $K$, i.e. the group homomorphism $\alpha: H \rightarrow \operatorname{Aut}(K)$ obeys $\alpha(h) k=k$ iff $h=e$ or $k=e$. Freeness implies the projection $h \mapsto \operatorname{Out}(K)$ is injective; such a semi-direct product $D=K \rtimes_{\alpha} H$ is called a Frobenius group. The twist $\omega \in H^{3}\left(B_{K} ; \mathbb{T}\right)$ should be compatible with $\alpha$ in the sense that it lies in the image of the natural map $H^{3}\left(B_{D} ; \mathbb{T}\right) \rightarrow H^{3}\left(B_{K} ; \mathbb{T}\right)$. The Haagerup subfactor is associated to $\left(\mathbb{Z}_{3}, \mathbb{Z}_{2}, \alpha(1)=-,[0]\right)$ and $D=S_{3}$.

Wildly Optimistic Guess. Let $(K, H, \alpha, \omega)$ be any quadruple defined above, corresponding to Frobenius group $D=K \rtimes_{\alpha} H$. There is an irreducible finite-depth subfactor associated to the pair $(K, H)$; the triple $(K, H, \alpha)$ is realised by a $Q$-system of endomorphisms. To any such quadruple there is a rational $\operatorname{VOA} \mathcal{V}(K, H, \alpha, \omega)$, and a completely rational net of subfactors realising the modular data of this twisted quantum double. This VOA is a generalised orbifold (controlled in some sense by $H$ ) of a holomorphic orbifold $\mathcal{V}_{K}$ by $K$. This VOA $\mathcal{V}_{K}$ also contains a holomorphic orbifold $\mathcal{V}_{D}$; both $\mathcal{V}(K, H, \alpha, \omega)$ and $\mathcal{V}_{D}$ contain a common rational VOA. For $K$ an odd cyclic group and $H=\mathbb{Z}_{2}$ acting by $n \mapsto-n$, this recovers the modular data given in Section 3.2 and also a subfactor of Izumi type $K$, i.e. a solution to equations (7.1)-(7.5) of [38]; the $K=\mathbb{Z}_{3}$ special case recovers the Haagerup subfactor.

This guess suggests that the Haagerup subfactor itself cannot be regarded as exotic. But this guess is in some control only for the odd cyclic case discussed above, where it seems quite plausible. The reason for requiring freeness is that this condition plays an important role in the derivation of $\mathcal{D}^{\omega} \mathrm{Hg}_{\nu}$ in Section 3.2, as well as in [38; probably it can be dropped but at a cost of complexity.

The possibility that the Haagerup subfactor is related in some way to $S_{3}$ appears to have been first made in [21]. The present paper clarifies, deepens, and generalises that relation. The remainder of this first section recalls modular data and modular invariants (Section 1.1), reviews the basic theory of subfactors and the tube algebra (Section 1.2), and discusses the modular data and character vectors of VOAs (Section 1.4). We also explain in Section 1.3 how to recover the original subfactor from its quantum double - unlike the rest of Section 1, this subsection contains original material. Section 2 develops the relation between the Haagerup subfactor and $S_{3}$. Section 3 puts the Haagerup modular data into a sequence and twists it. We also introduce the notion of grafting and discuss the role of affine so $\left(\nu^{2}+4\right)$ at level 2 . Section 4 relates our untwisted sequence to Izumi's hypothetical family of 3-star, 5star, 7-star, ... subfactors. Section 5 discusses VOA interpretations for our twisted sequence, and explains the Haagerup-dihedral diamond which generalises Section 2.

The Haagerup subfactor arose in Haagerup's classification [34] of irreducible finite depth subfactors of index between 4 and $3+\sqrt{3} \approx 4.73205$. Two other subfactors $N \subset M$ (both also regarded as exotic) appear there: the Asaeda-Haagerup subfactor with Jones index $(5+\sqrt{17}) / 2 \approx 4.56155$ [1], and the extended Haagerup subfactor [4] with index $\approx 4.37720$. Both seem unrelated to our family, and neither lies in a known sequence. As with the Haagerup subfactor, neither their $N-N$ nor $M-M$ systems are braided. Of course Questions 1 and 2 should also be asked of them, but no serious work can begin until the modular data of their doubles has been computed. 


\subsection{Modular data and modular invariants}

Modular data arises naturally in several contexts (for instance CFT, subfactors, and VOAs) - see [31] for a review. In CFT, modular invariants correspond to the 1-loop closed string partition function.

Definition 1. Modular data $(\Phi, 0, S, T)$ consists of a finite set $\Phi$, an element $0 \in \Phi$, and matrices $S=\left(S_{i, j}\right)_{i, j \in \Phi}$ and $T=\left(T_{i, j}\right)_{i, j \in \Phi}$, such that:

a) $S, T$ are unitary, $S$ is symmetric, $T$ is diagonal;

b) $S^{2}=(S T)^{3}, S^{4}=I$, the identity matrix;

c) $C:=S^{2}$ is a permutation matrix, and row 0 of $S$ consists of nonzero real numbers;

d) for all $i, j, k \in \Phi$, the following quantities are nonnegative integers:

$$
N_{i, j}^{k}:=\sum_{l \in \Phi} \frac{S_{i, l} S_{j, l} \overline{S_{k, l}}}{S_{0, l}} .
$$

We call modular data $(\Phi, 0, S, T)$ and $\left(\Phi^{\prime}, 0^{\prime}, S^{\prime}, T^{\prime}\right)$ equivalent if there is a bijection $\phi: \Phi \rightarrow \Phi^{\prime}$ such that $\phi(0)=0^{\prime}, S_{\phi(i), \phi(j)}^{\prime}=S_{i, j}$ and $T_{\phi(i), \phi(i)}^{\prime}=T_{i, i}$ for all $i, j \in \Phi$. The $i \in \Phi$ are called primaries and $0 \in \Phi$ is called the vacuum. The order 1 or 2 permutation $C$ is called charge-conjugation. $T_{0,0}=e^{-\pi 1 c / 12}$ for a real number $c$ (only defined mod 24 by the modular data) called the central charge. Equation (1.1) is called the Verlinde formula; the quantities $N_{i j}^{k}$ are called fusion coefficients and comprise the structure constants of a commutative associative algebra called the fusion ring. The coefficients $N_{i, j, k}:=N_{i, j}^{C i}$ are invariant under all 6 reorderings of indices. Also, $N_{0, i, j}=\delta_{i, C j}$.

Condition b) says that modular data generates a representation $\rho$ of the modular group $\mathrm{SL}_{2}(\mathbb{Z})$ through the assignment

$$
S=\rho\left(\begin{array}{cc}
0 & -1 \\
1 & 0
\end{array}\right), T=\rho\left(\begin{array}{ll}
1 & 1 \\
0 & 1
\end{array}\right) .
$$

Most examples considered in this paper have the additional property that $C=I$, i.e. $S$ is real and the representation is factors to $\mathrm{PSL}_{2}(\mathbb{Z})$. We assume throughout that the first row of $S$ is strictly positive - such modular data is called unitary.

From Definition 1 we obtain

$$
S_{i, j}=\bar{T}_{i, i} \bar{T}_{j, j} T_{0,0} \sum_{k \in \Phi} T_{k, k} S_{k, 0} N_{i, j}^{k}
$$

Thus if $T$ is known exactly but $S$ only approximately, (1.3) together with the integrality of the fusion coefficients (1.1) can be used to determine $S$ exactly (the quantumdimension $S_{i, 0} / S_{0,0}$ is the Perron-Frobenius eigenvalue of the matrix $N_{i}=\left(N_{i, j}^{k}\right)$, and $S_{0,0}>0$ is determined by the square $\sum_{i}\left(S_{i, 0} / S_{0,0}\right)^{2}$ of the global dimension $\left.1 / S_{0,0}\right)$. Equivalently, if $S, T$ and $S^{\prime}, T$ are both modular data sharing the same $T$, and $S^{\prime}$ is sufficiently close to $S$, then $S=S^{\prime}$. This is how we'll identify the modular data of the double of subfactors in Section 4.1, given numerical estimates of $S$. 
One consequence of this definition is that the entries of $S$ and $T$ lie in a cyclotomic extension $\mathbb{Q}\left[\xi_{L}\right]$ of the rationals (throughout this paper we write $\xi_{k}$ for $\exp [2 \pi \mathrm{l} / k])$. For any Galois automorphism $\sigma \in \operatorname{Gal}\left(\mathbb{Q}\left[\xi_{L}\right] / \mathbb{Q}\right) \cong \mathbb{Z}_{L}^{\times},(\Phi, 0, \sigma S, \sigma T)$ will be (generally inequivalent) modular data with identical fusion coefficients, where $\sigma$ acts entry-wise on $S$ and $T$. It can be shown [14] that for any $\sigma \in \operatorname{Gal}\left(\mathbb{Q}\left[\xi_{L}\right] / \mathbb{Q}\right)$, there is a permutation $j \mapsto \sigma j$ of $\Phi$ and choice $s_{\sigma}(j)$ of signs such that

$$
\sigma\left(S_{i, j}\right)=s_{\sigma}(i) S_{\sigma i, j}=s_{\sigma}(j) S_{i, \sigma j}
$$

An important class of examples of modular data comes from finite groups $G$. Write $e$ for the identity and $g^{h}$ for $h^{-1} g h$. Select a cocycle $\omega \in Z^{3}\left(B_{G} ; \mathbb{T}\right)$, where we write $\mathbb{T}$ for the unit circle in $\mathbb{C}$; the corresponding modular data depends up to equivalence only on its class in $H^{3}\left(B_{G} ; \mathbb{T}\right)$. Define

$$
\theta_{g}(h, l)=\omega\left(h, g^{h}, l\right) \bar{\omega}(g, h, l) \bar{\omega}\left(h, l, g^{h l}\right) .
$$

Then the restriction of each $\theta_{a}$ to the centraliser $\mathcal{Z}_{a}:=\{h \in G: h a=a h\}$ in $G$ is a normalised 2-cocycle. In this paper we are only interested in the cohomologically trivial case where all $\theta_{a}$ are coboundaries (this will happen for instance whenever the Schur multiplier $H^{2}\left(B_{\mathcal{Z}_{a}} ; \mathbb{T}\right)$ of each centraliser $\mathcal{Z}_{a}$ is trivial). This means that there exist 1-cochains $\epsilon_{a}: \mathcal{Z}_{a} \rightarrow \mathbb{T}$ for which $\epsilon_{a}(e)=1$ and both

$$
\begin{gathered}
\theta_{a}(h, g)=\left(\delta \epsilon_{a}\right)(h, g)=\epsilon_{a}(h) \epsilon_{a}(g) \bar{\epsilon}_{a}(h g), \\
\epsilon_{x^{-1} a x}\left(x^{-1} h x\right)=\theta_{a}\left(x, x^{-1} h x\right) \overline{\theta_{a}}(h, x) \epsilon_{a}(h),
\end{gathered}
$$

for all $g, h \in \mathcal{Z}_{a}$ and $x \in G$. The primaries are all pairs $(a, \pi)$ where $a$ runs through representatives of conjugacy classes in $G$ and $\pi \in \widehat{\mathcal{Z}_{a}}=\operatorname{Irr}\left(\mathcal{Z}_{a}\right)$ are irreducible representations (irreps). The vacuum is $(e, 1)$. The modular matrices are [16]

$$
\begin{gathered}
S_{(a, \chi),\left(a^{\prime}, \chi^{\prime}\right)}^{\omega}=\frac{1}{\left|\mathcal{Z}_{a}\right|\left|\mathcal{Z}_{a^{\prime}}\right|} \sum_{g \in G\left(a, a^{\prime}\right)} \bar{\chi}\left(g a^{\prime} g^{-1}\right) \bar{\chi}^{\prime}\left(a^{g}\right) \bar{\epsilon}_{a}\left(g a^{\prime} g^{-1}\right) \bar{\epsilon}_{g a^{\prime} g^{-1}}(a), \\
T_{(a, \chi),\left(a^{\prime}, \chi^{\prime}\right)}^{\omega}=\delta_{a, a^{\prime}} \delta_{\chi, \chi^{\prime}} \frac{\chi(a)}{\chi(e)} \epsilon_{a}(a)
\end{gathered}
$$

where $G\left(a, a^{\prime}\right)=\left\{g \in G: a^{g} b=b a^{g}\right\}$. In the following we let $\mathcal{D}^{\omega} G$ denote this modular data. See e.g. [16, 17, 15] for more details; we recover this formula next subsection using tube algebras.

Another class of modular data is associated to even positive-definite lattices. Let $L$ be such a lattice, and $n$ its dimension. Then $\Phi=L^{*} / L$ where $L^{*}$ is the dual of $L$. The vacuum 0 corresponds to the coset [0]. Then

$$
\begin{gathered}
S_{[u],[v]}=\left|L^{*} / L\right|^{-1 / 2} \exp [2 \pi \mathrm{r} u \cdot v], \\
T_{[u],[u]}=\exp [\pi \mathrm{\imath} u \cdot u-\pi \mathrm{r} n / 12] .
\end{gathered}
$$

The final class we need is associated to affine nontwisted Lie algebras $\mathfrak{g}^{(1)}$ and positive integers $k$ (see e.g. [42]). Let $\mathfrak{g}_{, k}$ denote the corresponding modular data: 
$\Phi$ is the set $P_{+}^{k}\left(\mathfrak{g}^{(1)}\right)$ of integrable highest-weights of level $k$, the vacuum is $k \Lambda_{0}$, and explicit formulas for the matrices $S$ and $T$ are given for instance in [42, Ch.13] or 31. Finite group, lattice and affine algebra modular data (and as we'll see next subsection, the double of a subfactor) is always unitary.

Definition 2. A matrix $Z=\left(Z_{i, j}\right)_{i, j \in \Phi}$ is called a modular invariant provided i) all entries $Z_{i, j}$ are nonnegative integers and $Z_{0,0}=1$;

ii) $Z S=S Z$ and $Z T=T Z$.

Examples are $Z=I$ and $Z=C$. A modular invariant is often written in equivalent form as the formal expression

$$
Z=\sum_{i, j \in \Phi} Z_{i, j} \operatorname{ch}_{i} \overline{c h}_{j}
$$

Unitary modular data has only finitely many modular invariants. The Galois symmetry (1.4) implies, for any $\sigma \in \operatorname{Gal}\left(\mathbb{Q}\left[\xi_{L}\right] / \mathbb{Q}\right)$,

$$
\begin{aligned}
& M_{i, j}=s_{\sigma}(i) s_{\sigma}(j) M_{\sigma i, \sigma j}, \\
& M_{i, j} \neq 0 \text { implies } s_{\sigma}(i)=s_{\sigma}(j) .
\end{aligned}
$$

\subsection{Subfactors, quantum doubles and tube algebras}

We refer to [41, 25] for the basic theory of subfactors, principal graphs etc, 48] for the theory of sectors, and [11, 9, 10] for the theory of alpha-induction.

Given a type III factor $N$, let ${ }_{N} \mathcal{X}_{N}$ denote a finite system of endomorphisms on $N$ [11, Defn 2.1]. Write $\Sigma\left({ }_{N} \mathcal{X}_{N}\right)$ for the endomorphisms which decompose into a finite number of irreducibles from ${ }_{N} \mathcal{X}_{N}$. For $\lambda, \rho \in \Sigma\left({ }_{N} \mathcal{X}_{N}\right)$, the intertwiner space $\operatorname{Hom}(\lambda, \rho)$ is a finite-dimensional Hilbert space. Write $\langle\lambda, \rho\rangle$ for its dimension. The sector $[\lambda]$ identifies all endomorphisms $\operatorname{Ad}(u) \lambda$ for unitaries $u$ in the target algebra.

Suppose ${ }_{N} \mathcal{X}_{N}$ is nondegenerately braided [11, Section 2.2]. Among other things this means $\lambda, \mu \in{ }_{N} \mathcal{X}_{N}$ commute up to a unitary $\epsilon=\epsilon(\lambda, \mu)$, i.e. $\lambda \mu=\operatorname{Ad}(\epsilon) \mu \lambda$, and these unitaries $\{\epsilon(\lambda, \mu)\}$ can be chosen to satisfy the braiding-fusion relations. Unitary modular data $S, T$ is obtained from this set-up by the intertwiners associated to the Hopf link and twist [55, 58]; here $\Phi={ }_{N} \mathcal{X}_{N}$ and 1 is the identity endomorphism, and the corresponding fusion ring is realised by composition in ${ }_{N} \mathcal{X}_{N}$ : $[\lambda][\mu]=\sum N_{[\lambda],[\mu]}^{[\nu]}[\nu]$ where $N_{[\lambda],[\mu]}^{[\nu]}=\langle[\lambda][\mu],[\nu]\rangle$.

Modular invariants are recovered as follows. Suppose we have a subfactor $N \subset M$. Let $\iota: N \rightarrow M$ be the inclusion and $\bar{\iota}: M \rightarrow N$ its conjugate. Then $\theta=\bar{\iota} \iota$ is called the canonical endomorphism and $\gamma=\iota \bar{\iota}$ its dual canonical endomorphism. Suppose $\theta$ is in $\Sigma\left({ }_{N} \mathcal{X}_{N}\right)$. Using the braiding $\epsilon^{+}:=\epsilon$ or its opposite $\epsilon^{-}:=\epsilon^{-1}$, we can lift an endomorphism $\lambda \in{ }_{N} \mathcal{X}_{N}$ of $N$ to one of $M: \alpha_{\lambda}^{ \pm}:=\gamma^{-1} \operatorname{Ad}\left(\epsilon^{ \pm}(\lambda, \theta)\right) \lambda \gamma$. Then $Z_{\lambda, \mu}:=\left\langle\alpha_{\lambda}^{+}, \alpha_{\mu}^{-}\right\rangle$is a modular invariant [11, 20]. The induced $\alpha^{ \pm}\left({ }_{N} \mathcal{X}_{N}\right)={ }_{M} \mathcal{X}_{M}^{ \pm}$ generate the full system ${ }_{M} \mathcal{X}_{M}$. The $N-M$ system ${ }_{N} \mathcal{X}_{M}$ (resp. the $M-N$ system $\left.{ }_{M} \mathcal{X}_{N}\right)$ consists of all irreducibles in $\iota \theta^{n}\left(\right.$ resp. $\left.\bar{\iota} \gamma^{n}\right)$. By the nimrep we mean the ${ }_{N} \mathcal{X}_{N}$ 
action on the $N-M$ system ${ }_{N} \mathcal{X}_{M}$. This is one of 8 (6 independent) natural products ${ }_{P} \mathcal{X}_{Q} \times{ }_{Q} \mathcal{X}_{R} \rightarrow{ }_{P} \mathcal{X}_{R}$ among the sectors, one for each triple $P, Q, R \in\{M, N\}$.

Many examples (e.g. the Haagerup and finite groups) arise naturally as nonbraided systems of endomorphisms. To get a braided system, one takes the quantum double (asymptotic inclusion) of ${ }_{N} \mathcal{X}_{N}$. This can be realised by the Longo-Rehren inclusion $A \subset B$ where $B=N \otimes N^{\text {opp }}$ (see e.g. [49, 37]): we are interested in the double $\mathcal{D}\left({ }_{N} \mathcal{X}_{N}\right)$ system on $A$ (which contains the $A$ - $A$ system of this subfactor, cf. Remark (i) p.146 of [37]) whereas the full system ${ }_{B} \mathcal{X}_{B}$ is simply ${ }_{N} \mathcal{X}_{N} \otimes{ }_{N} \mathcal{X}_{N}{ }^{\text {opp }}$ (see Theorem 1 below) with dual canonical endomorphism $\gamma_{L R}=\Sigma_{\xi \in_{N} \mathcal{X}_{N}} \xi \otimes \xi^{\mathrm{opp}}$.

If ${ }_{M} \mathcal{X}_{M}$ is an $M-M$ system of a subfactor $N \subset M$, then the dual Longo-Rehren inclusion is $\widehat{\iota}: A_{1} \subset M \otimes M^{\text {opp }}=: B_{1}$, where $\widehat{\gamma}=\overline{\widehat{\iota}}=\sum_{\eta \in_{M} \mathcal{X}_{M}} \eta \otimes \eta^{\text {opp }}$. Here $B_{1} \mathcal{X}_{B_{1}}$ is ${ }_{M} \mathcal{X}_{M} \otimes{ }_{M} \mathcal{X}_{M}^{\text {opp }}$ but we can and will identify the double $\mathcal{D}\left({ }_{N} \mathcal{X}_{N}\right)$ on $A$ with the double $\mathcal{D}\left({ }_{M} \mathcal{X}_{M}\right)$ on $A_{1}$, and $A_{1}$ with $A$.

The induction-restriction graph of the quantum double system is constructed in the following way [37]. First, given $\rho \in \Sigma\left({ }_{N} \mathcal{X}_{N}\right)$, a system of unitaries $\left\{\mathcal{E}_{\rho}(\xi)\right\}$ is called a half-braiding of $\rho$ if $\mathcal{E}_{\rho}(\xi) \in \operatorname{Hom}(\rho \xi, \xi \rho)$ for some $\xi \in{ }_{N} \mathcal{X}_{N}$, and $x \mathcal{E}_{\rho}(\zeta)=\xi\left(\mathcal{E}_{\rho}(\eta)\right) \mathcal{E}_{\rho}(\xi) \rho(x)$ for every $x \in \operatorname{Hom}(\zeta, \xi \eta)$, as in [37, Defn. 4.2]. This can be described by a matrix representation for an orthonormal basis $\left\{w_{\rho}(\xi)_{i}\right\}$ of $\operatorname{Hom}(\xi, \rho)$, if we set

$$
\mathcal{E}_{\rho}^{p}(\xi)_{(\eta, i),(\zeta, j)}:=\rho_{\xi}\left(w_{\rho}(\zeta)_{j}^{*}\right) \mathcal{E}_{\rho}^{p}(\xi) w_{\rho}(\eta)_{i} \in \operatorname{Hom}(\eta \xi, \xi \zeta)
$$

Then the even vertices of the quantum double system are labelled by inequivalent half-braidings $\mathcal{E}_{\rho}^{p}$, and the odd vertices are labelled by $\left(\xi \otimes \mathrm{id}^{\mathrm{opp}}\right) \iota$ with $\xi$ running in ${ }_{N} \mathcal{X}_{N}$. Finally vertices $\mathcal{E}_{\rho}$ and $\left(\xi \otimes \mathrm{id}^{\text {opp }}\right) \iota$ are joined by $\langle\rho, \xi\rangle$ edges, see [37, page 154]. The principal graph of $A \subset B$ is the connected component containing (id $\otimes$ id $\left.^{\text {opp }}\right) \iota$.

Incidentally, the forgetful functor sending the half-braiding $\mathcal{E}_{\rho}^{p}$ to the representation $\rho$ is an algebra homomorphism, and indeed has a name: it is alpha-induction. See also the discussion at the end of the next subsection.

The tube algebra is an effective way to compute the modular data $S, T$ of the quantum double. In Section 2 we compare the tube algebras of the Haagerup subfactor and the group $S_{3}$. Their similarity is the first indication of a possible relation between them. The tube algebra of ${ }_{N} \mathcal{X}_{N}$ is the finite dimensional $\mathrm{C}^{*}$-algebra

$$
\operatorname{Tube}\left({ }_{N} \mathcal{X}_{N}\right)=\bigoplus_{\xi, \eta, \zeta \in \in_{N} \mathcal{X}_{N}} \operatorname{Hom}(\xi \cdot \zeta, \zeta \cdot \eta)
$$

The simple summands of $\operatorname{Tube}\left({ }_{N} \mathcal{X}_{N}\right)$ are labelled by inequivalent half-braidings $\mathcal{E}_{\rho}^{p}$. The modular data $S, T$ is obtained from the half-braidings by (2.8),(2.9) of [38].

Let's consider in more detail the special case of tube algebras of finite groups. Let $G$ be a finite group with identity $e$. Given a type III factor $N$, write $\operatorname{Int}(N)$ for the group of inner automorphisms and $\operatorname{Out}(N):=\operatorname{Aut}(N) / \operatorname{Int}(N)$. Let $\alpha: G \rightarrow \operatorname{Out}(N)$ be a homomorphism. We take a lift $\alpha: G \rightarrow \operatorname{Aut}(N)$, as in [57].

Since $\left[\alpha_{g}\right]\left[\alpha_{h}\right]=\left[\alpha_{g h}\right]$ as sectors, there exists a unitary $u_{g, h} \in N$ satisfying $\alpha_{g} \cdot \alpha_{h}=\operatorname{Ad}\left(u_{g, h}\right) \alpha_{g h}$. In particular $u_{g, h} \in \operatorname{Hom}\left(\alpha_{g h}, \alpha_{g} \cdot \alpha_{h}\right)$. Associativity $\left(\alpha_{g} \alpha_{h}\right) \alpha_{k}=$ 
$\alpha_{g}\left(\alpha_{h} \alpha_{k}\right)$ implies $\operatorname{Ad}\left(u_{g, h}\right) u_{g h, k} \cdot \alpha_{g h k}=\operatorname{Ad}\left(\alpha_{g}\left(u_{h, k}\right) u_{g, h k}\right) \cdot \alpha_{g h k}$. Then since $N$ is a factor, there exists a scalar $\omega(g, h, k) \in \mathbb{T}$ satisfying $u_{g, h} u_{g h, k}=\omega(g, h, k) \alpha_{g}\left(u_{h, k}\right) u_{g, h k}$, i.e. $\omega$ is an element in $Z^{3}\left(B_{G} ; \mathbb{T}\right)$. Conversely, every element in $Z^{3}\left(B_{G} ; \mathbb{T}\right)$ arises as such an $\omega$ [57, 40].

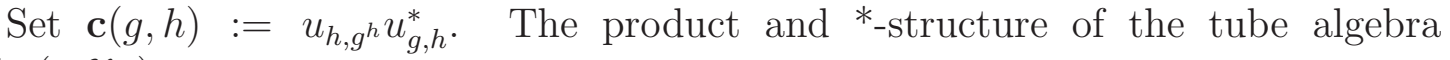
$\operatorname{Tube}\left({ }_{N} \mathcal{X}_{N}\right)$ are:

$$
\begin{aligned}
& \mathbf{c}(g, h) \mathbf{c}(k, l)=\delta_{k, g^{h}} \theta_{g}(h, l) \mathbf{c}(g, h l) \\
& \mathbf{c}(g, h)^{*}=\omega\left(g h, h^{-1}, h\right) \omega\left(h^{-1}, g h, h^{-1}\right) \omega\left(h^{-1}, h, g^{h}\right) \mathbf{c}\left(g^{h}, h^{-1}\right)
\end{aligned}
$$

(recall (1.5) $)$. Consequently,

$$
\mathbf{c}(e, g) \mathbf{c}(e, h)=\mathbf{c}(e, g h) \text { and } \mathbf{c}(e, g)^{*}=\mathbf{c}\left(e, g^{-1}\right)
$$

and thus the group algebra $\mathbb{C}[G]$ of $G$ is a $\mathrm{C}^{*}$-subalgebra of $\operatorname{Tube}\left({ }_{N} \mathcal{X}_{N}\right)$. The identity of the tube algebra is $\sum_{g \in G} \mathbf{c}(g, e)$.

Let $K_{g}=\left\{g^{h}: h \in G\right\}$ denote the conjugacy class of an element $g \in G$. Then we have the decomposition $\operatorname{Tube}\left({ }_{N} \mathcal{X}_{N}\right)=\bigoplus_{K_{u}} \operatorname{Tube}\left(K_{u}\right)$ where the sum ranges over the conjugacy classes of $G$, and $\operatorname{Tube}\left(K_{u}\right)=\bigoplus_{g \in K_{u}, l \in G} \operatorname{Hom}\left(\alpha_{g} \alpha_{l}, \alpha_{l} \alpha_{g^{l}}\right)$. Hence $\mathbb{C}[G]=\operatorname{Tube}\left(K_{e}\right)$.

This decomposition can be further refined. Consider first a trivial twist $\omega$ [28]. The outer action $\alpha$ gives a subfactor $N \subset N \rtimes G=M$. The $N-N$ system is $\left\{\alpha_{g}\right\} \equiv G$ whereas the $M-M$ system is the irreps $\widehat{G}$. The tube algebras $\operatorname{Tube}(G) \simeq \operatorname{Tube}(\widehat{G})$ are Morita equivalent as finite-dimensional $\mathrm{C}^{*}$-algebras [37], so their centres, the quantum doubles of $G$ and $\widehat{G}$, are identified. The simple components of Tube $(G)$, equivalently the even vertices of the quantum double of $G$, are labelled by pairs $\left(K_{u}, \pi\right)$ where $\pi \in \widehat{\mathcal{Z}_{u}}$ are irreps of the centralizers $\mathcal{Z}_{u}$, see e.g. [46] or [26, Sect. 4]. To every such pair $\left(K_{u}, \pi\right)$ is attached the endomorphism $\rho_{\left(K_{u}, \pi\right)} \in \Sigma(G)$ defined as

$$
\rho_{\left(K_{u}, \pi\right)}=\operatorname{dim}(\pi) \sum_{h \in K_{u}} \alpha_{h}
$$

In the dual system $\widehat{G}$, the endomorphism $\hat{\rho}_{\left(K_{u}, \pi\right)}$ in $\Sigma(\widehat{G})$ attached to such a pair $\left(K_{u}, \pi\right)$ is the Mackey induction $\operatorname{Ind}_{\mathcal{Z}_{u}}^{G} \pi$ from $\mathcal{Z}_{u}$ to $G$, decomposed into irreps of $G$.

The number of inequivalent half-braidings associated to $\rho_{\left(K_{u}, \pi\right)}$ equals the number of inequivalent irreps $\pi^{\prime} \in \widehat{\mathcal{Z}_{u}}$ such that $\operatorname{dim}\left(\pi^{\prime}\right)=\operatorname{dim}(\pi)$. The half-braiding for $\rho=\rho_{\left(K_{u}, \pi\right)}$ in its matrix decomposition is

$$
\mathcal{E}_{\rho}^{\pi}(\xi)_{\left(u^{p}, i\right),\left(u^{q}, j\right)}=\pi_{j i}\left(p \xi^{-1} q^{-1}\right) \operatorname{id}_{N},
$$

for all $\xi \in p^{-1} \mathcal{Z}_{u} q, u^{p}, u^{q} \in K_{u}$, where $i, j$ label the basis vectors in the representation space of $\pi$.

All this extends to the twisted case. The $\theta_{a}$ 's of (1.5) are normalised twisted cocycles on $G$, namely from (1.14) they satisfy

$$
\theta_{a}(x, y) \theta_{a}(x y, z)=\theta_{a}(x, y z) \theta_{x^{-1} a x}(y, z), \quad \forall x, y, z \in G .
$$


They thus describe projective representations of $\mathcal{Z}_{a}$. As we know [17], the primary fields in the model twisted by a given 3 -cocycle $\omega$ consist of all pairs $\left(K_{a}, \tilde{\pi}\right)$ where now $\tilde{\pi} \in \theta_{a}-\operatorname{Irr}\left(\mathcal{Z}_{a}\right)$.

In the cohomologically trivial case discussed in Section 1.1, this is immediate: we can twist the formula (1.17) for half-braidings by $\epsilon$; inserting into $(2.8),(2.9)$ of [38] recovers (1.8).

\subsection{The canonical and dual canonical modular invariants}

This subsection contains original material.

Suppose we have a subfactor $N \subset M$, with $N-N$ system denoted by $\Delta$ and $M-M$ system $\widehat{\Delta}$. The double $\mathcal{D} \Delta=L R(\Delta)$ of $\Delta$ is realised as sectors on a factor $A$ in $N \otimes N^{\text {opp }}=: B$. Alpha-induction reverses this. That is, if you take the appropriate modular invariant $Z_{\Delta}$ in the double, and take alpha-induction from $A$ to $B$, then the full system is $\Delta \otimes \Delta^{\mathrm{opp}}$. In some sense then, the factor $A$ and the modular invariant $Z_{\Delta}$ remembers the original system $\Delta$ on $A$. In fact it is a consequence of Theorem 1 below that the $A-B$ system is just $\Delta$ regarded as a nimrep.

To get $B$, only the canonical endomorphism $\theta$ on $A$ is needed (because of Theorem 1 below, we can read $\theta$ off from the modular invariant), plus the associated Q-system on $\theta$. That is, all the information about $B$ and the full system $\Delta \otimes \Delta^{\text {opp }}$ is carried in the primary fields and modular data of the double, the modular invariant $Z_{\Delta}$ and its vacuum block $\theta$. We suggest this modular invariant $Z_{\Delta}$ be called the canonical modular invariant.

Dually, there is an inclusion $A \subset M \otimes M^{\text {opp }}=: B$, where the dual $\widehat{\Delta}$ acts on $M$, but the same double acts on $A$. This comes with another modular invariant $Z_{\widehat{\Delta}}$, with a corresponding $\widehat{\theta}$ which regains $\widehat{\Delta} \otimes \widehat{\Delta}^{\text {opp }}$ as the full system. Here (again from Theorem 1 below) the $A$ - $B$ system is just $\widehat{\Delta}$. Again the modular invariant $Z_{\widehat{\Delta}}$ - the dual canonical modular invariant — and $\widehat{\theta}$ together encode the original system $\widehat{\Delta}$.

This means we should regard the double $\mathcal{D} \Delta \cong \mathcal{D} \widehat{\Delta}$ as equipped with two canonical modular invariants $Z_{\Delta}$ and $Z_{\widehat{\Delta}}$, from which using say alpha-induction one can recover the full system $B-B$ and the nimrep $A-B$ entirely in terms of $\Delta$ or $\widehat{\Delta}$. In particular the full system is $\Delta \otimes \Delta^{\mathrm{opp}}$ or $\widehat{\Delta} \otimes \widehat{\Delta}^{\mathrm{opp}}$, depending on the choice of modular invariant $Z_{\Delta}$ or $Z_{\widehat{\Delta}}$, and the nimrep is $\Delta$ or $\widehat{\Delta}$.

Note that in both cases the $A-B$ system here is an algebra. For most modular invariants the $A-B$ system is only a module, but when the inclusion is type I, which implies for instance that the modular invariant is a sum of squares, the $A$ - $B$ system will necessarily be an algebra $(A \subset B$ is type $\mathrm{I}$ iff the equivalent conditions of Proposition 3.2 in [8] hold; in the nets of subfactors setting $A(I) \subset B(I)$, this means the extended net is local). This suggests that both inclusions $A \subset N \otimes N^{\text {opp }}$ and $A \subset M \otimes M^{\text {opp }}$ are type I. In fact more is true:

Theorem 1. The inclusions $A \subset N \otimes N^{\text {opp }}$ and $A \subset M \otimes M^{\text {opp }}$ are both type $I$. Moreover, we have $\left(Z_{\Delta}\right)_{i, j}=\left(Z_{\Delta}\right)_{i, 0}\left(Z_{\Delta}\right)_{j, 0}$, i.e. $Z_{\Delta}=\mid \sum_{i} Z_{i, 0}$ ch $\left.\right|^{2}$ (similarly for 
$\left.Z_{\widehat{\Delta}}\right)$. The vectors $u$ and $v$ with entries $u_{i}=\left(Z_{\Delta}\right)_{i, 0}$ and $v_{i}=\left(Z_{\widehat{\Delta}}\right)_{i, 0}$ are eigenvectors with eigenvalue 1 of both $S$ and $T$.

Proof. Corollaries 6.3 and 6.4 of [37] show that, at the level of subfactors, ${ }_{B} \mathcal{X}_{B}^{+}:=$ $\left.\alpha^{+}{ }_{B} \mathcal{X}_{B}\right) \subseteq \Delta \otimes 1$ and ${ }_{B} \mathcal{X}_{B}^{-}:=\alpha^{-}\left({ }_{A} \mathcal{X}_{A}\right) \subseteq 1 \otimes \Delta^{\text {opp }}$. The neutral system ${ }_{B} \mathcal{X}_{B}^{0}=$ ${ }_{B} \mathcal{X}_{B}^{+} \cap{ }_{B} \mathcal{X}_{B}^{-}$is thus contained in $\Delta \otimes 1 \cap 1 \otimes \Delta^{\text {opp }}$ and hence equals 1 . But $\operatorname{dim}_{B} \mathcal{X}_{B}^{ \pm}$ is computed in [8, Prop.3.1], for such a nondegenerate system like ours, to be

$$
\frac{1}{S_{0,0}}=\sum \frac{S_{i, 0}}{S_{0,0}} Z_{i, 0}=\sum \frac{S_{j, 0}}{S_{0,0}} Z_{0, j}
$$

which matches the dimensions of $\Delta \otimes 1$ and $1 \otimes \Delta^{\mathrm{opp}}$. Therefore we get the equalities ${ }_{B} \mathcal{X}_{B}^{+}=\Delta \otimes 1$ and ${ }_{B} \mathcal{X}_{B}^{-}=1 \otimes \Delta^{\text {opp }}$.

Any canonical endomorphism $\theta$ is bounded below by the vacuum sector: $\theta \geq$ $\sum_{\lambda} Z_{\lambda, 0} \lambda$. This inequality is a special case of $\langle\theta \lambda, \mu\rangle \geq\left\langle\alpha_{\lambda}^{ \pm}, \alpha_{\mu}^{ \pm}\right\rangle$([12, eq.(37)]; see also [6, Thm.3.9]). But $\operatorname{dim} \theta$ is given by

$$
\operatorname{dim} \theta=\operatorname{dim}(\overline{\iota \iota})=\operatorname{dim}(\iota \bar{\iota})=\sum \operatorname{dim}\left(\xi \otimes \xi^{\mathrm{opp}}\right)=\sum(\operatorname{dim} \xi)^{2}=\frac{1}{S_{0,0}},
$$

agreeing with (1.18), and thus $\theta=\sum_{\lambda} Z_{\lambda, 0} \lambda$. By [8, Prop.3.2], this implies the inclusion $A \subset B$ is type I.

Because of alpha-induction, we know a modular invariant takes the form $Z=$ $\sum_{\tau, \lambda, \mu} b_{\tau, \lambda}^{+} b_{\tau, \mu}$, where $\tau$ runs over all sectors of the neutral system and $b_{\tau, \lambda}^{ \pm}=\left\langle\tau, \alpha_{\lambda}^{ \pm}\right\rangle$ are the branching coefficients. Because this system is type I, we have $b^{+}=b^{-}$. Because the neutral system is trivial, we have only one $\tau$. Thus the modular invariant $Z_{\Delta}$ corresponding to $\theta$, and $Z_{\widehat{\Delta}}$ corresponding to $\widehat{\theta}$, take the desired forms. The statements about the vectors $u, v$ follow from modular invariance. QED

From the theory of alpha-induction, we know that $\Delta$ (respectively $\widehat{\Delta}$ ) is noncommutative iff some $\left(Z_{\Delta}\right)_{i, j}>1$ (resp. some $\left.\left(Z_{\widehat{\Delta}}\right)_{i, j}>1\right)$ - see [11, Cor.6.9],[12, Thm.4.11]. For type I inclusions, we know from Section 4.1 of [7] that the $A$ - $B$ system ${ }_{A} \mathcal{X}_{B}$ is isomorphic to ${ }_{B} \mathcal{X}_{B}^{ \pm}$.

We will call any modular invariant of the type $Z=\left|\sum_{i} Z_{i, 0} c h_{i}\right|^{2}$ a monomial modular invariant.

Of course all this applies for a system $\Delta$ of endomorphisms not necessarily coming from a subfactor - we expect this is relevant for our twisted Haagerup data. Again $\Delta$ is recovered from the double $\mathcal{D} \Delta$ and the canonical modular invariant $Z_{\Delta}$, which again is monomial. The only difference is that there is no $\widehat{\Delta}$.

Suppose a finite group $G$ acts by outer automorphism on a type III factor $N$. Then as mentioned last subsection, by taking a crossed product we have a subfactor $N \subset M$ where the $N-N$ and $M-M$ systems are identified with $G$ and $\widehat{G}$ respectively. Taking the Longo-Rehren inclusion $A \subset N \otimes N^{\mathrm{opp}}$, the doubled $A$-A system can be identified with the untwisted quantum double $\mathcal{D} G$. We will sometimes find it useful to use the K-theory language developed in [22, 23], which identifies $\mathcal{D} G$ with the equivariant K-group $K_{G}^{0}(G) \cong K_{\Delta-\Delta}^{0}(G \times G)$ where $G$ acts on $G$ by the conjugate action and 
$\Delta=\Delta(G) \cong G$ acts diagonally on the left and right of $G \times G$. The neutral system is trivial $\left(K^{0}(e, e)=\mathbb{Z}\right)$, with sigma-restriction $\sigma_{\text {id }}=\theta_{\mathcal{D} G} \cong \sum_{\chi \in \widehat{G}} \operatorname{dim} \chi(e, \chi)$. The full system is $\Delta \times \Delta^{\mathrm{opp}} \cong K^{0}(G \times G)$ with canonical modular invariant

$$
Z_{\Delta}=\left|\sum \chi(e) \operatorname{ch}_{(e, \chi)}\right|^{2},
$$

the sum over $\chi \in \widehat{G}$. The dual LR-inclusion is $A \subset M \otimes M^{\text {opp }}$, where the doubled $A$ - $A$ system is again the quantum double of $G$, the full system is $\widehat{G} \times \widehat{G}^{\text {opp }} \cong K_{G \times G}^{0}(G / G \times$ $G / G)$ and the neutral system $K^{0}(G / G \times G / G)$ is trivial. Here sigma-restriction is given by $\widehat{\sigma}_{\text {id }}=\widehat{\theta}_{\mathcal{D} G} \cong \sum(g$,id $)$ where $g$ runs over representatives of all conjugacy classes, and the dual canonical modular invariant is

$$
Z_{\widehat{\Delta}}=\left|\sum \operatorname{ch}_{(a, 1)}\right|^{2}
$$

where the sum runs over representatives $a$ of all conjugacy classes of $G$. We recover from (1.19), (1.20) that $\widehat{\Delta}=\widehat{G}$ is always commutative, whereas $\Delta=\mathbb{C} G$ is commutative iff all dimensions $\chi(e)=1$, i.e. iff $G$ is abelian.

By the Galois theory of [37, a subgroup $K<G$ induces an intermediate subfactor $A \subset C \subset N \otimes N^{\text {opp }}$ where the doubled $C$ - $C$ system is the untwisted quantum double $\mathcal{D} K \cong K_{K}^{0}(K) \cong K_{\Delta K-\Delta K}^{0}(K \times K)$. Then $A \subset C$ is a braided subfactor of index $|G / K|$. The full system ${ }_{C} \mathcal{X}_{C} \cong K_{\Delta K-\Delta K}^{0}(G \times G)$ and chiral systems ${ }_{C} \mathcal{X}_{C}^{ \pm} \cong{ }_{C} \mathcal{X}_{A} \cong$ $K_{\Delta K-\Delta G}^{0}(G \times G)$. The branching coefficients and sigma-restriction $K_{K}^{0}(K) \rightarrow K_{G}^{0}(G)$ are given by $(g, \pi) \rightarrow \Sigma\left(g^{\prime}, \operatorname{Ind}_{K \cap Z_{g}}^{Z_{g}} \pi\right)$, where the summation is over equivalence classes of $g^{\prime}$ in $G$ conjugate to $g$. The trivial example $K=1$ recovers the canonical modular invariant (1.19). In the case of the dihedral group $G=D_{\nu}$ and its cyclic subgroup $K=\mathbb{Z}_{\nu}$, in which we are interested, the canonical modular invariant arising from the inclusion is given in (3.11) below.

\subsection{VOAs and vector-valued modular functions}

For the basic theory of VOAs, see [47]. Let $\mathcal{V}$ be a VOA. Write $\operatorname{Irr}(\mathcal{V})$ for the set of irreducible $\mathcal{V}$-modules $M$. Among other things, $\mathcal{V}$ and its modules carry a representation of the Virasoro algebra $\operatorname{Vir}=\operatorname{Span}\left\{L_{n}, C\right\}_{n \in \mathbb{Z}}$, where the central term $C$ acts as a scalar $c=c(\mathcal{V})$ called the central charge. $c$ also arose in Section 2.1, where it was defined only mod 24 . The action of $L_{0} \in \operatorname{Vir}$ on each $\mathcal{V}$-module $M$ defines a grading (by eigenvalues) on $M$ into finite-dimensional spaces.

Definition 3. A VOA $\mathcal{V}$ is called rational if

i) $\mathcal{V} \in \operatorname{Irr}(\mathcal{V})$ and $\mathcal{V}$ is isomorphic to its contragredient as $\mathcal{V}$-modules;

ii) writing $\mathcal{V}=\oplus_{n \in \mathbb{Z}} \mathcal{V}_{n}$ for the grading by $L_{0}$ of $\mathcal{V}$, we have $\mathcal{V}_{n}=0$ for $n<0$ and $\mathcal{V}_{0}$ is 1-dimensional;

iii) every weak $\mathcal{V}$-module is completely reducible.

See [47] for the details, which play no role in the following. There is no standard definition of rationality - we chose Definition 3 to guarantee the existence of modular data. All VOAs considered in this paper are rational in this sense. 
Rational VOAs $\mathcal{V}$ (or if you prefer, rational CFT) realise modular data as follows. The primaries consist of the finitely many modules $M \in \operatorname{Irr}(\mathcal{V})=\Phi$, and the vacuum 0 is $\mathcal{V}$ itself. Define their characters to be the graded dimensions

$$
c h_{M}(\tau)=\operatorname{Tr}_{M} q^{L_{0}-c / 24}
$$

using the above grading by $L_{0}$, where as always we write $q=e^{2 \pi 1 \tau}$. These $c h_{M}$ will be holomorphic throughout the upper half-plane $\mathbb{H}=\{\tau \in \mathbb{C}: \operatorname{Im}(\tau)>0\}$ 62. Collect these finitely many characters into a column vector $c \vec{h}(\tau)$. Then 62 showed there is a representation $\rho$ of $\mathrm{SL}_{2}(\mathbb{Z})$ such that

$$
\overrightarrow{c h}\left(\frac{a \tau+b}{e \tau+d}\right)=\rho\left(\begin{array}{ll}
a & b \\
e & d
\end{array}\right) \overrightarrow{c h}(\tau), \forall\left(\begin{array}{ll}
a & b \\
e & d
\end{array}\right) \in \mathrm{SL}_{2}(\mathbb{Z}) .
$$

The matrices $S, T$ now defined by (1.2) constitute modular data [36]. It may or may not be unitary. Unlike braided subfactors, which are naturally associated to modular invariants, VOAs see only one chiral half of the rational CFT, and so capture only the notion of modular data.

There is a rational VOA $\mathcal{V}(L)$ corresponding to any even positive-definite lattice $L$ (the central charge equals the dimension $n$ of $L$ ), recovering the modular data of (1.9). The character corresponding to coset $[v] \in L^{*} / L$ is $c h_{[v]}(\tau)=\eta(\tau)^{-n} \sum_{x \in[v]} q^{x \cdot x / 2}$. There is a rational VOA $\mathcal{V}\left(\mathfrak{g}_{k}\right)$ corresponding to affine algebra $\mathfrak{g}^{(1)}$ at positive integral level $k$, recovering the modular data of [42. The character $c h_{\lambda}$ corresponding to highest-weight $\lambda$ coincides with the affine algebra character $\chi_{\lambda}$, specialised to $\tau \in \mathbb{H}$. Affine algebra and lattice VOAs overlap for the simply-laced $\mathfrak{g}$ at level 1 , corresponding to the root lattices $L$. Finite group modular data $\mathcal{D}^{\omega} G$ is recovered by taking the orbifold $\mathcal{V}^{G}$ (i.e. subVOA of fixed-points of $G$ ) of a subgroup $G$ of automorphisms of a holomorphic VOA (i.e. a rational $\operatorname{VOA} \mathcal{V}$ with $\operatorname{Irr}(\mathcal{V})=\{\mathcal{V}\}$ ) - it has been conjectured that any such $\mathcal{V}^{G}$ will itself be rational, but a general proof of this still seems far away. Examples of holomorphic VOAs are the $\mathcal{V}(L)$ for $L$ self-dual - e.g. for $c=8$ there is the $E_{8}$ root lattice. In this case the orbifold theory is under better control, see e.g. [43], and indeed this is the main one we'll consider. A sexier example of holomorphic VOA though is the $c=24$ Moonshine module $V^{\natural}$.

Incidentally, Theorem 1 suggests that: a VOA corresponding to a quantum double should be a conformal subalgebra of a holomorphic VOA.

Definition 4. Let $\rho$ be a d-dimensional representation of $S L_{2}(\mathbb{Z})$, with diagonal $T:=$ $\rho\left(\begin{array}{l}11 \\ 01\end{array}\right)$. A weakly holomorphic vector-valued modular function $\overrightarrow{c h}(\tau)$ with multiplier $\rho$ is a holomorphic function $\mathbb{H} \rightarrow \mathbb{C}^{d}$ satisfying (1.22), with q-expansion

$$
\overrightarrow{c h}(\tau)=q^{\lambda} \sum_{n=0}^{\infty} \overrightarrow{c h}_{n} q^{n}
$$

for some diagonal matrix $\lambda$ satisfying $T=e^{2 \pi 1 \lambda}$, where each Fourier coefficient $\overrightarrow{c h}_{n} \in$ $\mathbb{C}^{d}$ is independent of $\tau$. Let $\mathcal{M}(\rho)$ denote the space of all such $\overrightarrow{c h}$ for $\rho$. 
Equation (1.23) means $\overrightarrow{c h}(\tau)$ is meromorphic at the cusp. [62] showed that the character vector of a rational VOA is a vector-valued modular function in this sense. These character vectors will help us study and identify the VOA.

The characters of rational VOAs also satisfy other conditions. For instance, by definition (1.21) the Fourier coefficients $c \vec{h}_{n}$ in (1.23) are nonnegative integers. The vacuum character $c h_{\mathcal{V}}=c h_{0}$ begins with $1 q^{-c / 24}$. In a unitary VOA, $\lambda$ in (1.23) can be chosen so that $\lambda_{M}>-c / 24$ for all $M \neq \mathcal{V}$ in $\operatorname{Irr}(\mathcal{V})$.

Recall charge-conjugation $C=S^{2}$ from Definition 1. A consequence of (1.22) is that $C \overrightarrow{c h}=\overrightarrow{c h}$. For convenience we assume in the remainder of this subsection that $C=I$, i.e. we have a representation of $\mathrm{PSL}_{2}(\mathbb{Z})$. This holds for most modular data considered in this paper (other than $\mathcal{D}^{\omega} \mathbb{Z}_{2 n+1}$ ), but at the end of Section 2.4 we explain how to reduce $C \neq I$ to the $C=I$ case.

Given any $d$-dimensional $\mathrm{PSL}_{2}(\mathbb{Z})$-representation $\rho$, 2, 3] describe how to find all $c \vec{h}(\tau) \in \mathcal{M}(\rho)$. In particular, there is a $d \times d$ matrix $\Xi(\tau)$ with the property that $\overrightarrow{c h}(\tau) \in \mathcal{M}(\rho)$ iff $\overrightarrow{c h}(\tau)$ is of the form $\vec{X}(\tau)=\Xi(\tau) \vec{P}(J(\tau))$, where $\vec{P}(x)$ is any column vector whose entries are polynomials in $x$, and $J(\tau)$ is the Hauptmodul $J(\tau)=q^{-1}+196884 q+\cdots$. Thus it suffices to find $\Xi(\tau)$ for $\rho . \Xi(\tau)$ is determined through a differential equation it satisfies. This differential equation and the relevant initial condition is determined from two $d \times d$ matrices of numbers. One is a diagonal matrix $\Lambda$ satisfying among other things the relation $e^{2 \pi 1 \Lambda}=T$; the more elusive one is called $\chi$. In terms of $\Lambda$ and $\chi$, the matrix $\Xi(\tau)$ has $q$-expansion

$$
\Xi(\tau)=q^{\Lambda} \sum_{n=-1}^{\infty} q^{n} \Xi_{n}=q^{\Lambda}\left(I q^{-1}+\chi+\sum_{n=1}^{\infty} q^{n} \Xi_{n}\right),
$$

where $\Xi_{-1}$ is the $d \times d$ identity matrix, $\Xi_{0}$ is the matrix $\chi$, and for $n=1,2,3, \ldots$ the matrix $\Xi_{n}$ is recursively defined by the commutator

$$
\left[\Lambda, \Xi_{n}\right]+(n+1) \Xi_{n}=\sum_{l=-1}^{n-1} \Xi_{l}\left(f_{n-l}(\Lambda-I)+g_{n-1}(\chi+[\Lambda, \chi])\right),
$$

where $f_{n}, g_{n}$ are defined by $(J-240) \Delta / E_{10}=\sum f_{n} q^{n}$ and $\Delta / E_{10}=\sum g_{n} q^{n}$ for the discriminant form $\Delta=\eta^{24}$ and Eisenstein series $E_{10}=E_{4} E_{6}$. That is, the $(i, j)$-entry of $\Xi_{n}$ is the $(i, j)$-entry of (1.24), divided by $\Lambda_{i i}-\Lambda_{j j}+n+1$.

The following method suffices to find $\Lambda, \chi$ for the $\rho$ considered in this paper (though invariably there are more elegant methods). First, decompose $\rho$ into irreps. What is special about irreps $\rho$ is that their $\mathcal{M}(\rho)$ is cyclic (Theorem 4.1 of [3]): the space $\mathcal{M}(\rho)$ is a module over the ring $\mathbb{C}\left[J, \nabla_{1}, \nabla_{2}, \nabla_{3}\right]$ of differentiable operators

$$
\nabla_{1}=\frac{E_{10}}{\Delta} \mathrm{D}_{0}, \nabla_{2}=\frac{E_{8}}{\Delta} \mathrm{D}_{1} \mathrm{D}_{0}, \nabla_{3}=\frac{E_{6}}{\Delta} \mathrm{D}_{2} \mathrm{D}_{1} \mathrm{D}_{0}, \text { for } \mathrm{D}_{k}=q \frac{\mathrm{d}}{\mathrm{d} q}-\frac{k}{6} E_{2},
$$

and if $\rho$ is irreducible, then $\mathcal{M}(\rho)$ (and hence $\Lambda$ and $\chi$ ) is generated over that ring by any nonzero $\overrightarrow{c h} \in \mathcal{M}(\rho)$. All irreps occurring in this paper are subrepresentations 
of the modular data coming from even lattices, and so the desired nonzero modular function can be built from lattice theta functions.

Knowing $\Lambda$ and $\chi$ for a given $S, T$ is equivalent to knowing $\Lambda$ and $\chi$ for $S, \omega T$ for any third root of unity $\omega$, but the explicit equivalence [3] is not easy. Write $\Xi^{(k)}(\tau)$ for the matrix $\Xi(\tau)$ for $S, \xi_{3}^{k} T$ and assume $\Xi^{(0)}(\tau)$ (hence $\Lambda^{(0)}$ and $\chi^{(0)}$ ) is known. Then the columns of $\Xi^{(1)}$ are linear combinations over $\mathbb{C}$ of the columns of $\eta^{-16} E_{4}^{2} \Xi^{(0)}$ and $\eta^{-16}\left(E_{6} \mathrm{D}_{0} \Xi^{(0)}-E_{4}^{2} \Xi^{(0)}\left(\Lambda^{(0)}-1\right)\right)$, while the columns of $\Xi^{(2)}$ are linear combinations over $\mathbb{C}$ of $\eta^{-8} E_{4} \Xi^{(0)}$ and $\eta^{-8}\left(\mathrm{D}_{1} \mathrm{D}_{0} \Xi^{2}-E_{4} \Xi^{(0)}\left(\Lambda^{(0)}-I\right)\left(\Lambda^{(0)}-\frac{7}{6} I\right)\right)$.

The contragredient $\left(\rho^{T}\right)^{-1}$ is handled similarly [3]: the columns of $E_{10} E_{14} \Delta^{-2}\left(\Xi^{T}\right)^{-1}$ are linearly independent vectors in $\mathcal{M}\left(\left(\rho^{T}\right)^{-1}\right)$.

\section{Comparing the Haagerup, Sym(3) and $\mathrm{SO}(13)$}

\subsection{The tube algebra of $\operatorname{Sym}(3)$}

Recall the discussion in Section 1.2. Write $S_{3}=\operatorname{Sym}(3)=\left\{e, u, u^{2}, \tau, \tau u, \tau u^{2}\right\}$. The three conjugacy classes are $K_{e}=\{e\}, K_{u}=\left\{u, u^{2}\right\}$ and $K_{\tau}=\left\{\tau, \tau u, \tau u^{2}\right\}$. Write $\widehat{S}_{3}=\{1, \epsilon, \sigma\}$ where $\epsilon$ is sgn and $\sigma$ is 2-dimensional. Then each pair $\left(K_{g}, \pi\right)$, with $\pi \in \widehat{\mathcal{Z}}_{g}$, gives rise to a simple component of the tube algebra.

There are two half-braidings for endomorphism $\rho=e$ as $\rho_{\left(K_{e}, \mathbf{1}\right)}=\rho_{\left(K_{e}, \epsilon\right)}=e$, and a third attached to $\rho_{\left(K_{e}, \sigma\right)}=e+e$. We denote these half-braidings by $e^{(1)}, e^{(2)}$ and $2 e$, respectively. The conjugacy class $K_{u}$ provides an endomorphism $\rho_{\left(K_{u}, \pi\right)}=$ $u+u^{2}$ for every irrep $\pi$ of the centraliser $\mathcal{Z}_{u} \simeq \mathbb{Z}_{3}$, and hence three half-braidings $\left(u+u^{2}\right)^{(1)},\left(u+u^{2}\right)^{(2)},\left(u+u^{2}\right)^{(3)}$. The conjugacy class $K_{\tau}$ has centraliser $\mathcal{Z}_{\tau} \simeq \mathbb{Z}_{2}$, so the endomorphism $\rho=\tau+\tau u+\tau u^{2}$ has two half-braidings $\left(\tau+\tau u+\tau u^{2}\right)^{(1)}$, $\left(\tau+\tau u+\tau u^{2}\right)^{(2)}$.

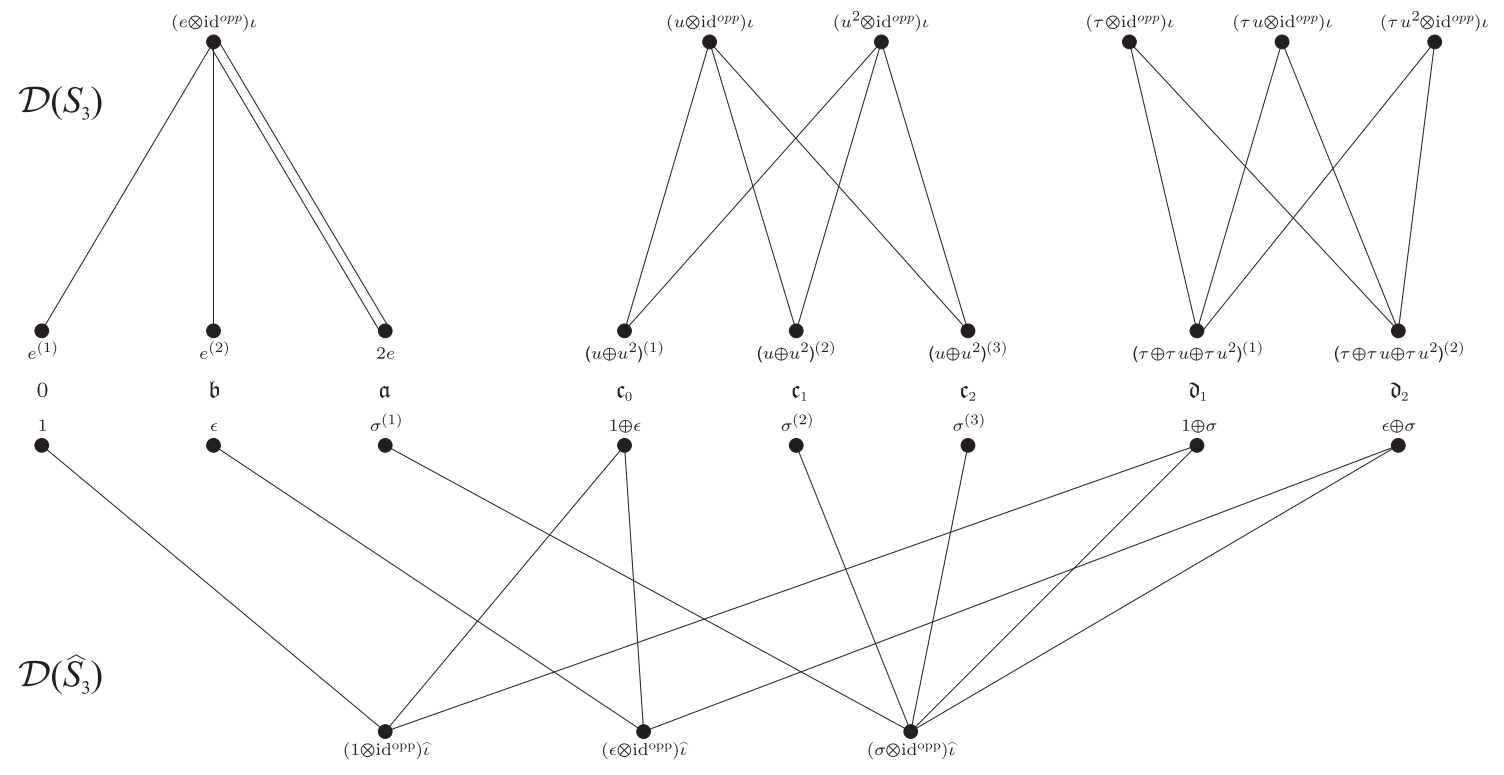

Figure 1. Dual principal graphs for doubles of $\operatorname{Sym}(3)$ and $\widehat{\operatorname{Sym}(3)}$ 
This enables us to match up Figure 1, where the bottom graph has been drawn in [38, Fig. 1] or [26, Fig. 31]: the upper graph arises from the Longo-Rehren inclusion of $S_{3}$, and the lower describes the Longo-Rehren inclusion of the dual system $\widehat{S}_{3}$, see [37, Remark (i) in Page 154] as well as Section 1.2 above for the general description of the induction-restriction graphs between $A-B$ and $B-B$ sectors of the Longo-Rehren inclusion $A \subset B$ using the structure of the tube algebra. The middle vertices in Figure 1 also describe how the half-braidings from $S_{3}$ and $\widehat{S}_{3}$ match up.

The Longo-Rehren dual sectors associated to $S_{3}$ and $\widehat{S}_{3}$ are respectively

$$
\begin{gathered}
{\left[\theta_{\mathcal{D} S_{3}}\right]=\left[K_{e}, \operatorname{Ind}_{\mathrm{id}}^{\widehat{G}}\right]=\sum_{\pi \in \widehat{G}} \operatorname{dim}(\pi)\left[K_{e}, \pi\right]=\left[\epsilon^{(1)}\right]+\left[\epsilon^{(2)}\right]+2[2 \epsilon],} \\
{\left[\theta_{\mathcal{D S}_{3}}\right]=\sum_{g}\left[K_{g}, \operatorname{Ind}_{K_{g}}^{G} \mathrm{id}\right]=[1]+[1+\epsilon]+[1+\sigma],}
\end{gathered}
$$

specialised from the discusson of Section 1.3. Observe that $\left[\theta_{\mathcal{D} \widehat{S}_{3}}\right]$ has also been computed in [38, 26]. These braided subfactors yield the canonical modular invariants (recall (1.19),(1.20) $) Z_{22}=\left|c h_{0}+c h_{\mathfrak{b}}+2 c h_{\mathfrak{a}}\right|^{2}$ and $Z_{55}=\left|c h_{0}+c h_{\mathfrak{c}_{0}}+c h_{\mathfrak{d}_{1}}\right|^{2}$ with full systems $S_{3} \times S_{3}^{\text {opp }}, \widehat{S}_{3} \times{\widehat{S_{3}}}^{\text {opp }}$ respectively by [26, page 357], where the names of the primaries of $\mathcal{D} S_{3}$ are taken from the middle row of Figure 1 and the names $Z_{22}, Z_{55}$ come from the list in [26].

As explained at the end of Section 1.3 , the subsystem $\mathbb{Z}_{3}$ of $\Delta$ corresponds to an intermediate subfactor $A \subset C \subset N \otimes N^{\text {opp }}$ where the dual canonical endomorphism of $C \subset N \otimes N^{\text {opp }}$ is $\gamma=\sum_{\alpha \in \mathbb{Z}_{3}} \alpha \otimes \alpha^{\text {opp }}$ and the double $C-C$ system is the quantum double $\mathcal{D} \mathbb{Z}_{3} \cong K_{\mathbb{Z}_{3}}^{0}\left(\mathbb{Z}_{3}\right)$. Then $A \subset C$ is a braided subfactor of index 2 , with canonical endomorphism $[\theta]=[0]+[\mathfrak{b}]$ and associated modular invariant

$$
Z=\left|c h_{0}+c h_{\mathfrak{b}}\right|^{2}+2\left|c h_{\mathfrak{a}}\right|^{2}+2\left|c h_{\mathfrak{c}_{0}}\right|^{2}+2\left|c h_{\mathfrak{c}_{1}}\right|^{2}+2\left|c h_{\mathfrak{c}_{2}}\right|^{2} .
$$

\subsection{The Haagerup tube algebra}

Let $\Delta=\left\{\mathrm{id}, u, u^{2}, \rho, \rho u, \rho u^{2}\right\}$ be the noncommutative $N-N$ system (the even vertices of the top graph of Figure 2) of the Haagerup subfactor $N \subset M$ of index $\delta+1$, where $\delta=(3+\sqrt{13}) / 2$. The fusions (product of sectors) are given by:

$$
[u]^{3}=[\mathrm{id}], \quad[u][\rho]=[\rho][u]^{2}, \quad[\rho]^{2}=[\mathrm{id}]+[\rho]+[\rho u]+\left[\rho u^{2}\right] .
$$

These have statistical dimensions $\operatorname{dim}(u)=1$ and $\operatorname{dim}(\rho)=\delta$. Let $\widehat{\Delta}=\{$ id, $a, b, c\}$ be the commutative $M-M$ system (the even vertices of the bottom graph of Figure 2), whose fusion rules are:

$$
\begin{aligned}
{[a]^{2}=[\mathrm{id}]+[a]+[b]+[c], } & {[b]^{2}=[\mathrm{id}]+[c], \quad[c]^{2}=[\mathrm{id}]+2[a]+[b]+2[c], } \\
{[a][b]=[a]+[c], } & {[a][c]=[a]+[b]+2[c], \quad[b][c]=[a]+[b]+[c] . }
\end{aligned}
$$

Hence we obtain the statistical dimensions $\operatorname{dim}(a)=\delta, \operatorname{dim}(b)=\delta-1, \operatorname{dim}(c)=\delta+1$. 


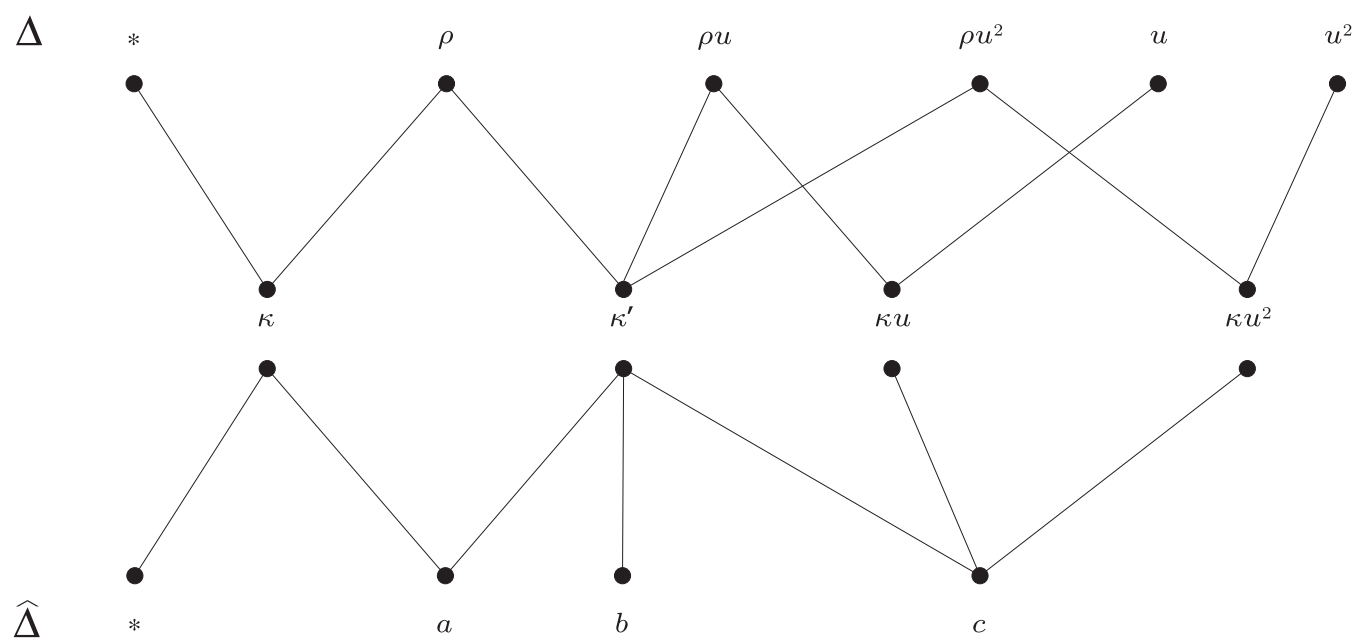

Figure 2. Principal graphs of the Haagerup $(5+\sqrt{13}) / 2$ subfactor

The $M-N$ sectors, $\left[\kappa u^{i}\right]$ and $\left[\kappa^{\prime}\right]$ where $\kappa$ is the inclusion $N \subset M$, are the odd vertices of both graphs in Figure 2. From this figure we see we can choose the endomorphism $c=\kappa u \bar{\kappa} \cong \kappa u^{2} \bar{\kappa}$. These principal graphs encode multiplication by $\kappa-$ e.g. $[\kappa]\left[\rho u^{i}\right]=\left[\kappa^{\prime}\right]+\left[\kappa u^{i}\right]$. From this we obtain the statistical dimensions $\operatorname{dim}\left(\kappa u^{i}\right)=\lambda$ and $\operatorname{dim}\left(\kappa^{\prime}\right)=\lambda(\delta-1)$. The remaining products $M-N \times N-N \rightarrow M-N$ and $M-M \times M-N \rightarrow M-N$, as well as $M-N \times N-M \rightarrow M-M$ and $N-M \times M-N \rightarrow N-$ $N$, were computed by Bisch [5] and are generalised in Section 4.2 below.

The fusions of $\Delta$ and $\widehat{\Delta}$ should remind one of $S_{3}$. In particular, the system $\Delta$ has $\mathbb{Z}_{3}=\left\{\mathrm{id}, u, u^{2}\right\}$ as a subsystem and is some sort of perturbation of the usual $S_{3}$ multiplication table $[u]^{3}=[\mathrm{id}], \quad[u][\rho]=[\rho][u]^{2}, \quad[\rho]^{2}=[\mathrm{id}]$. Similarly, the $\widehat{\Delta}$ system reduces to the character ring $\widehat{S}_{3}$ when ignoring $[c]$, where $b$ should be regarded as $S_{3}$-representation $\epsilon$ and $a$ as $\sigma$. These similarities were our first indication of a relation between the Haagerup subfactor and $S_{3}$. We find in Section 4 that this $\Delta \leftrightarrow S_{3}$ relation generalises naturally, whereas $\widehat{\Delta} \leftrightarrow \widehat{S}_{3}$ does not.

The structure of the tube algebra of the system $\Delta$ has been studied in [38, Sect. 8]. Izumi introduced the following endomorphisms in $\Sigma(\Delta)$ :

$$
\mu=\rho+\rho u+\rho u^{2}, \quad \pi_{1}=\mathrm{id}+\mu, \quad \pi_{2}=2(\mathrm{id})+\mu, \quad \sigma=u+u^{2}+\mu,
$$

and proved that $\pi_{1}$ and $\pi_{2}$ have only one half-braiding, $\sigma$ has three $\left(\sigma^{(1)}, \sigma^{(2)}, \sigma^{(3)}\right)$, and finally $\mu$ has six $\left(\mu^{(1)}, \ldots, \mu^{(6)}\right)$ and that these half-braidings exhaust all of them, so that the quantum double of $\Delta$ has 12 primaries as in the middle row of Figure 3. The induction-restriction graph of the Longo-Rehren inclusion of the system $\Delta$ is given in [38, Fig. 5], see also the top of Figure 3. From this we read off the LongoRehren dual sector associated to $\Delta$, namely $\left[\theta_{\mathcal{D} \Delta}\right]=[0]+[\mathfrak{b}]+2[\mathfrak{a}]$, using the labelling of Figure 3. This corresponds to the canonical modular invariant (recall Section 1.3) $Z_{22}=\left|\chi_{0}+\chi_{\mathfrak{b}}+2 \chi_{\mathfrak{a}}\right|^{2}$, with corresponding full system $\Delta \times \Delta^{\text {opp }}$, where the name $Z_{22}$ comes from the list of [27] (see also Section 3.4 below). From Figure 3 we obtain the quantum dimensions $1+3 \delta, 2+3 \delta, 2+3 \delta, 3 \delta$ for $\mathfrak{b}, \mathfrak{a}, \mathfrak{c}_{j}, \mathfrak{d}_{l}$, respectively. 


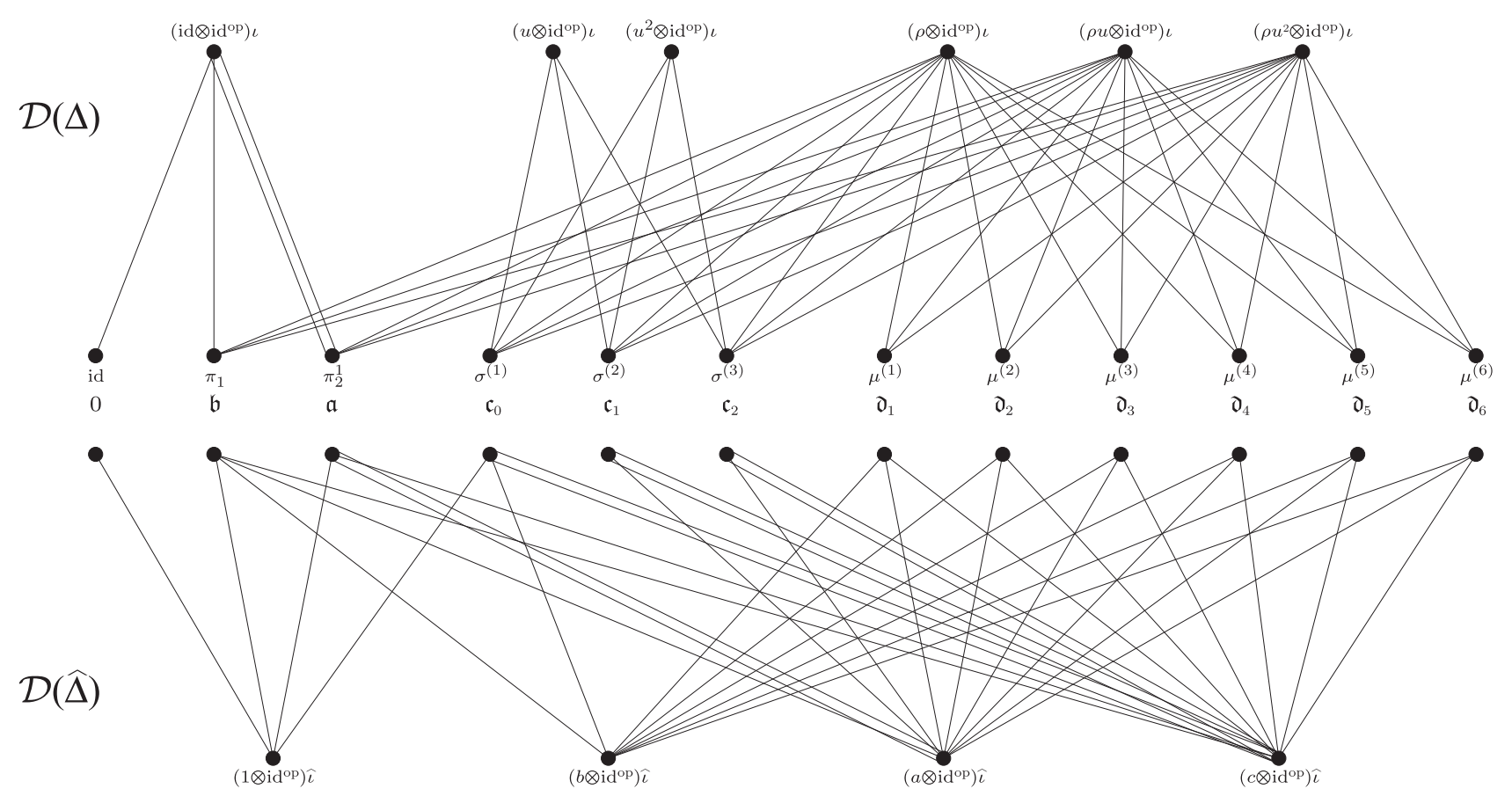

Figure 3. Dual principal graphs for doubles of $\Delta$ and $\widehat{\Delta}$ for Haagerup

More subtle is the dual principal graph corresponding to $\widehat{\Delta}$, on the bottom of Figure 3, with dual canonical endomorphism $\left[\theta_{\mathcal{D} \widehat{\Delta}}\right]=[0]+[\mathfrak{b}]+[\mathfrak{a}]+\left[\mathfrak{c}_{0}\right]$ and modular invariant $Z_{11}=\left|\chi_{0}+\chi_{\mathfrak{b}}+\chi_{\mathfrak{a}}+\chi_{\mathfrak{c} 0}\right|^{2}$ corresponding to $\widehat{\Delta} \times \widehat{\Delta}^{\text {opp }}$. To our knowledge this appears here for the first time. To derive it, first note that the only monomial modular invariants for $\mathcal{D} H g$ are $Z_{22}=\left|c h_{0}+c h_{\mathfrak{b}}+2 c h_{\mathfrak{a}}\right|^{2}, Z_{11}=\left|c h_{0}+c h_{\mathfrak{b}}+c h_{\mathfrak{a}}+c h_{\mathfrak{c}_{0}}\right|^{2}$, and $Z_{33}=\left|c h_{0}+c h_{\mathfrak{b}}+2 c h_{\mathfrak{c}_{0}}\right|^{2}$ (Proposition 4 below with $\nu=3$ ). The number of sectors in $\widehat{\Delta}$ is 4 , and the canonical endomorphism $\gamma_{\widehat{\Delta}}=0 \otimes 0^{\text {opp }}+a \otimes a^{\text {opp }}+b \otimes b^{\text {opp }}+c \otimes c^{\text {opp }}$. Since $\left\langle\gamma_{\widehat{\Delta}}, \gamma_{\widehat{\Delta}}\right\rangle=4$, we have $\langle\widehat{\theta}, \widehat{\theta}\rangle=4$ by Frobenius reciprocity. Thus the canonical modular invariant $Z$ associated to $\widehat{\theta}$ satisfies $\sum_{i} Z_{0, i}^{2}=4$, forcing it to be $Z_{11}$ and fixing $\widehat{\theta}$. Hence $\widehat{\Delta}$ has $1^{2}+1^{2}+1^{2}+1^{2}=4$ irreducible sectors, call them $e_{0}, e_{1}, e_{2}, e_{3}$, one of which (say $e_{0}$ ) is the identity $\widehat{\alpha}_{0}$. To obtain the dual principal graph, in which $e_{i}$ is connected to the primary $x$ with $\left\langle\widehat{\alpha}_{e_{i}}, x\right\rangle$ edges, use $\left\langle\widehat{\alpha}_{x}, \widehat{\alpha}_{y}\right\rangle=\langle\widehat{\theta} x, y\rangle$, which holds for any type I $\widehat{\theta}$ (see Theorem 1). The fusions for the double Haagerup are explicitly given in [27] (or Section 3.2 below). The edges from $\mathfrak{a}$ are determined from the calculations $\left\langle\widehat{\alpha}_{\mathfrak{a}}, \widehat{\alpha}_{\mathfrak{a}}\right\rangle=6=1^{2}+1^{2}+2^{2}$ and $\left\langle\widehat{\alpha}_{\mathfrak{a}}, \widehat{\alpha}_{0}\right\rangle=1$ : call $e_{1}$ the sector not adjacent to $\mathfrak{a}$, and $e_{2}$ the one connected to $\mathfrak{a}$ with 2 edges. The additivity of the statistical dimension $\operatorname{dim} \widehat{\alpha}_{\mathfrak{a}}=\operatorname{dim} \mathfrak{a}$ then identifies $e_{1}=b, e_{2}=a, e_{3}=c$. Likewise, the edges from $\mathfrak{b}$ come from $\left\langle\widehat{\alpha}_{\mathfrak{b}}, \widehat{\alpha}_{\mathfrak{b}}\right\rangle=4$ and $\left\langle\widehat{\alpha}_{\mathfrak{b}}, \widehat{\alpha}_{0}\right\rangle=1$, and those from $\mathfrak{d}_{l}$ come from $\left\langle\widehat{\alpha}_{\mathfrak{d}_{l}}, \widehat{\alpha}_{\mathfrak{d}_{l}}\right\rangle=3$ and $\left\langle\widehat{\alpha}_{\mathfrak{d}_{l}}, \widehat{\alpha}_{0}\right\rangle=0$. From $\left\langle\widehat{\alpha}_{\mathfrak{c}_{j}}, \widehat{\alpha}_{\mathfrak{c}_{j}}\right\rangle=5+\delta_{j, 0},\left\langle\widehat{\alpha}_{\mathfrak{c}_{j}}, \widehat{\alpha}_{0}\right\rangle=\delta_{j, 0}$, and statistical dimensions, we obtain the final edges.

We have $A \subset N \otimes N^{\text {opp }}$, the double of $\Delta$, with subsystem $\mathbb{Z}_{3} \subset \Delta$. Hence by the Galois theory of Izumi [37, we have as in Section 1.3 and last subsection an intermediate subfactor $A \subset C \subset N \otimes N^{\mathrm{opp}}$ where the dual canonical endomorphism 
of $C \subset N \otimes N^{\text {opp }}$ is $\gamma=\sum_{\alpha \in \mathbb{Z}_{3}} \alpha \otimes \alpha^{\text {opp }}$ and the double $C$-C system is the quantum double $\mathcal{D Z}_{3} \cong K_{\mathbb{Z}_{3}}^{0}\left(\mathbb{Z}_{3}\right)$. Then $A \subset C$ is a braided subfactor of index $2+3 \delta$, with canonical endomorphism $[\theta]=[0]+[\mathfrak{b}]$ and associated modular invariant (recall (2.3))

$$
Z=\left|c h_{0}+c h_{\mathfrak{b}}\right|^{2}+2\left|c h_{\mathfrak{a}}\right|^{2}+2\left|c h_{\mathfrak{c}_{0}}\right|^{2}+2\left|c h_{\mathfrak{c}_{1}}\right|^{2}+2\left|c h_{\mathfrak{c}_{2}}\right|^{2} .
$$

\subsection{Haagerup modular data $\mathcal{D H g}$}

The modular data $\mathcal{D} H g$ for the quantum double of the even part of the Haagerup subfactor was first computed in [38] and simplified somewhat in [27]. It is necessary for all that follows though to significantly simplify it further. The result is

$$
\begin{aligned}
& T=\operatorname{diag}\left(1,1,1,1, \xi_{3}, \overline{\xi_{3}}, \xi_{13}^{6}, \xi_{13}^{-2}, \xi_{13}^{2}, \xi_{13}^{5}, \xi_{13}^{-6}, \xi_{13}^{-5}\right) \text {, } \\
& S=\frac{1}{3}\left(\begin{array}{cccccccccccc}
x & 1-x & 1 & 1 & 1 & 1 & y & y & y & y & y & y \\
1-x & x & 1 & 1 & 1 & 1 & -y & -y & -y & -y & -y & -y \\
1 & 1 & 2 & -1 & -1 & -1 & 0 & 0 & 0 & 0 & 0 & 0 \\
1 & 1 & -1 & 2 & -1 & -1 & 0 & 0 & 0 & 0 & 0 & 0 \\
1 & 1 & -1 & -1 & -1 & 2 & 0 & 0 & 0 & 0 & 0 & 0 \\
1 & 1 & -1 & -1 & 2 & -1 & 0 & 0 & 0 & 0 & 0 & 0 \\
y & -y & 0 & 0 & 0 & 0 & c(1) & c(2) & c(3) & c(4) & c(5) & c(6) \\
y & -y & 0 & 0 & 0 & 0 & c(2) & c(4) & c(6) & c(5) & c(3) & c(1) \\
y & -y & 0 & 0 & 0 & 0 & c(3) & c(6) & c(4) & c(1) & c(2) & c(5) \\
y & -y & 0 & 0 & 0 & 0 & c(4) & c(5) & c(1) & c(3) & c(6) & c(2) \\
y & -y & 0 & 0 & 0 & 0 & c(5) & c(3) & c(2) & c(6) & c(1) & c(4) \\
y & -y & 0 & 0 & 0 & 0 & c(6) & c(1) & c(5) & c(2) & c(4) & c(3)
\end{array}\right),
\end{aligned}
$$

for $x=(13-3 \sqrt{13}) / 26, y=3 / \sqrt{13}$ and $c(j)=-2 y \cos (2 \pi j / 13)$.

It is important to note that the last 6 diagonal entries of $T$ are $T_{\mathfrak{D}_{l}, \mathrm{o}_{l}}=\xi_{13}^{6 l^{2}}$ for $1 \leq l \leq 6$, and the bottom-right $6 \times 6$ submatrix has entries $S_{\mathfrak{o}_{l}, \mathfrak{d}^{\prime}}=c\left(l l^{\prime}\right) / 3$ for $1 \leq l, l^{\prime} \leq 6$. Our expression for that $6 \times 6$ submatrix is considerably simpler than the corresponding expressions in [38, 27, 35]. The proof of the equivalence is easy, e.g. use (1.3). A direct derivation is given in Theorem 5 below.

$\mathcal{D H g}$ should be compared to the modular data $\mathcal{D} S_{3}$ for the (untwisted) double of $S_{3}$, which can be obtained from (1.8) (or see (3.1) below). As would be anticipated from the previous subsection, they are very similar, except that $x$ becomes $1 / 2$ and the last 6 rows/columns collapse into 2 .

The given matrix $T$ forces central charge $c$ to be a multiple of 24 (since $T_{0,0}=1$ ), but multiplying $T$ by a third root of unity (and leaving $S$ unchanged) allows us to consider the Haagerup at any multiple $c$ of 8 . Next section we generalise $\mathcal{D} H g$ in two ways: it can be twisted by a $\mathbb{Z}_{3}$ (this twist is analogous to the $H^{3}\left(B_{G} ; \mathbb{T}\right.$ )-twist of finite group modular data, or the level $k \in \mathbb{Z}_{>0}$ of affine algebra modular data); and it lies in an infinite sequence corresponding to the odd dihedrals.

\subsection{Clarifying the Haagerup-Sym(3) relation}

To emphasise that the relations between $S_{3}$ and the Haagerup aren't spurious, let us consider possible character vectors realising these modular data (recall Section 1.4). This will also help identify a VOA realisation. In this subsection we focus on the 
simplest possibility, central charge $c=8$ (the smallest possible), although similar results hold for any other multiple of 8 .

Consider first the modular data $\mathcal{D} S_{3}$ for the quantum double of $S_{3}$, with primaries $0, \mathfrak{b}, \mathfrak{a}, \mathfrak{c}_{i}, \mathfrak{d}_{l}$ as in Figure 1. This 8-dimensional $\mathrm{PSL}_{2}(\mathbb{Z})$-representation decomposes into 3 copies of the 1-dimensional irrep with $T=e^{-2 \pi_{1} / 3}$, a 2-dimensional irrep with kernel the principal congruence subgroup $\Gamma(2)$, and a 3-dimensional irrep with kernel containing $\Gamma(3)$. These 1-, 2-, and 3-dimensional irreps are also subrepresentations of the modular data for the lattices $E_{8}, D_{8}$ and $A_{2} \oplus E_{6}$ respectively, and as explained at the end of Section 1.4 the corresponding theta functions provide all the information needed to extract $\Lambda$ and $\chi$ for these irreps. From this we obtain for $\mathcal{D} S_{3}$ at $c \equiv_{24} 8$ the matrices $\Lambda_{\bar{S} 3}^{8}=\operatorname{diag}(2 / 3,2 / 3,2 / 3,2 / 3,0,1 / 3,2 / 3,1 / 6)$ and

$$
\chi_{\bar{S} 3}=\left(\begin{array}{cccccccc}
39 & 47 & 81 & 81 & 8748 & 1215 & 128 & 5120 \\
47 & 39 & 81 & 81 & 8748 & 1215 & -128 & -5120 \\
81 & 81 & 167 & -81 & -8748 & -1215 & 0 & 0 \\
81 & 81 & -81 & 167 & -8748 & -1215 & 0 & 0 \\
3 & 3 & -3 & -3 & -12 & 18 & 0 & 0 \\
27 & 27 & -27 & -27 & 1458 & -152 & 0 & 0 \\
128 & -128 & 0 & 0 & 0 & 0 & 120 & -5120 \\
16 & -16 & 0 & 0 & 0 & 0 & -16 & 140
\end{array}\right)
$$

(throughout the paper we write $a \equiv_{n} b$ for $\left.a \equiv b(\bmod n)\right)$.

Once we know $\Lambda, \chi$, then the simple recursion (1.24) yields the full $q$-expansion of $\Xi(\tau)$. From $\Xi$ we know completely explicitly all possible weakly holomorphic vectorvalued modular functions for this $\mathrm{SL}_{2}(\mathbb{Z})$-representation. Specialising to $c=8$, we find there is a unique possible character vector, namely the first column of $\Xi \overline{\bar{S}} 8$ :

$$
\left(\begin{array}{c}
c h_{0}(\tau) \\
c h_{\mathfrak{b}}(\tau) \\
c h_{\mathfrak{a}}(\tau)=c h_{\mathfrak{c}_{0}}(\tau) \\
c h_{\mathfrak{c}_{1}}(\tau) \\
c h_{\mathfrak{c}_{2}}(\tau) \\
c h_{\mathfrak{o}_{1}}(\tau) \\
c h_{\mathfrak{d}_{2}}(\tau)
\end{array}\right)=\left(\begin{array}{c}
q^{-1 / 3}\left(1+39 q+699 q^{2}+5761 q^{3}+35593 q^{4}+\cdots\right) \\
q^{2 / 3}\left(47+671 q+5825 q^{2}+35459 q^{3}+\cdots\right) \\
81 q^{2 / 3}\left(1+17 q+143 q^{2}+877 q^{3}+\cdots\right) \\
3+243 q+2916 q^{2}+21870 q^{3}+\cdots \\
27 q^{1 / 3}\left(1+22 q+221 q^{2}+1476 q^{3}+\cdots\right) \\
128 q^{2 / 3}\left(1+16 q+136 q^{2}+832 q^{3}+\cdots\right) \\
16 q^{1 / 6}\left(1+36 q+394 q^{2}+2776 q^{3}+\cdots\right)
\end{array}\right) .
$$

It is easy to realise $\mathcal{D} S_{3}$ (and hence the character vector (2.6)) at $c=8$. Start with the lattice $\operatorname{VOA} \mathcal{V}\left(E_{8}\right)$ corresponding to the $E_{8}$ root lattice. Its automorphism group is the compact Lie group $E_{8}(\mathbb{R})$. Then the VOA realising (2.6) is the orbifold of $\mathcal{V}\left(E_{8}\right)$ by some subgroup $G$ of $E_{8}(\mathbb{R})$ isomorphic to $S_{3}$ (i.e. is the subVOA of $\mathcal{V}\left(E_{8}\right)$ fixed by $G$ ). This is indeed possible (see Theorem 4.3 of [43]).

Now turn to the double of the Haagerup, also at $c=8$, with primaries labelled as in Figure 3. Its 12-dimensional $\mathrm{PSL}_{2}(\mathbb{Z})$-representation decomposes into $1+1+3+7$, where the 1- and 3-dimensional irreps are as before, and the 7-dimensional irrep occurs as a subrepresentation of the modular data of the 4-dimensional lattice $A_{3} 52_{1}[1,1 / 4]$, in the gluing notation of Conway-Sloane [13]. From this we quickly obtain for $c \equiv_{24} 8$ 
its matrices $\Lambda$ and $\chi$ :

$$
\begin{aligned}
& \operatorname{diag}(2 / 3,2 / 3,2 / 3,2 / 3,0,1 / 3,5 / 39,20 / 39,32 / 39,2 / 39,8 / 39,11 / 39) \\
& \left(\begin{array}{cccccccccccc}
6 & 80 & 81 & 81 & 8748 & 1215 & 3549 & 273 & 13 & 5538 & 2275 & 1378 \\
80 & 6 & 81 & 81 & 8748 & 1215 & -3549 & -273 & -13 & -5538 & -2275 & -1378 \\
81 & 81 & 167 & -81 & -8748 & -1215 & 0 & 0 & 0 & 0 & 0 & 0 \\
81 & 81 & -81 & 167 & -8748 & -1215 & 0 & 0 & 0 & 0 & 0 & 0 \\
3 & 3 & -3 & -3 & -12 & 18 & 0 & 0 & 0 & 0 & 0 & 0 \\
27 & 27 & -27 & -27 & 1458 & -152 & 0 & 0 & 0 & 0 & 0 & 0 \\
7 & -7 & 0 & 0 & 0 & 0 & -88 & -14 & -1 & 50 & 63 & 64 \\
42 & -42 & 0 & 0 & 0 & 0 & -1484 & 92 & 16 & 2940 & -192 & -1041 \\
119 & -119 & 0 & 0 & 0 & 0 & -2142 & 987 & 11 & -24990 & -6035 & 4641 \\
5 & -5 & 0 & 0 & 0 & 0 & 17 & 13 & -3 & -2 & 35 & -14 \\
13 & -13 & 0 & 0 & 0 & 0 & 174 & -1 & -5 & 294 & -147 & 51 \\
14 & -14 & 0 & 0 & 0 & 0 & 448 & -77 & 7 & -343 & 125 & -24
\end{array}\right)
\end{aligned}
$$

As before, this gives us the full $q$-expansion of $\Xi_{\bar{h} g} 8$.

There are only two possible character vectors for the Haagerup modular data at $c=8$, namely $\gamma=0$ or $\gamma=1$ in

$$
\left(\begin{array}{c}
c h_{0}(\tau) \\
c h_{\mathfrak{b}}(\tau) \\
c h_{\mathfrak{a}}(\tau)=c h_{\mathfrak{c}_{0}}(\tau) \\
\operatorname{ch}_{\mathfrak{c}_{1}}(\tau) \\
\operatorname{ch}_{\mathfrak{c}_{2}}(\tau) \\
\operatorname{ch}_{\mathfrak{d}_{1}}(\tau) \\
\operatorname{ch}_{\mathfrak{d}_{2}}(\tau) \\
\operatorname{ch}_{\mathfrak{d}_{3}}(\tau) \\
\operatorname{ch}_{\mathfrak{d}_{4}}(\tau) \\
\operatorname{ch}_{\mathfrak{d}_{5}}(\tau) \\
\operatorname{ch}_{\mathfrak{d}_{6}}(\tau)
\end{array}\right)=\left(\begin{array}{c}
q^{2 / 3}\left(q^{-1}+(6+13 \gamma)+(120+78 \gamma) q+(956+351 \gamma) q^{2}+(6010+1235 \gamma) q^{3}+\cdots\right) \\
q^{2 / 3}\left((80-13 \gamma)+(1250-78 \gamma) q+(10630-351 \gamma) q^{2}+(65042-1235 \gamma) q^{3}+\cdots\right) \\
q^{2 / 3}\left(81+1377 q+11583 q^{2}+71037 q^{3}+\cdots\right) \\
3+243 q+2916 q^{2}+21870 q^{3}+\cdots \\
q^{1 / 3}\left(27+594 q+5967 q^{2}+39852 q^{3}+\cdots\right) \\
q^{5 / 39}\left((7-\gamma)+(292-6 \gamma) q+(3204-43 \gamma) q^{2}+(23010-146 \gamma) q^{3}+\cdots\right) \\
q^{20 / 39}\left((42+16 \gamma)+(777+121 \gamma) q+(7147+547 \gamma) q^{2}+(45367+2000 \gamma) q^{3}+\cdots\right) \\
q^{32 / 39}\left(\gamma q^{-1}+(11 \gamma+119)+(73 \gamma+1623) q+(300 \gamma+12996) q^{2}+(76429+1063 \gamma) q^{3}+\cdots\right) \\
q^{2 / 39}\left((5-3 \gamma)+(229-50 \gamma) q+(2738-252 \gamma) q^{2}+(19942-1032 \gamma) q^{3}+\cdots\right) \\
q^{8 / 39}\left((13-5 \gamma)+(347-37 \gamma) q+(3804-212 \gamma) q^{2}+(26390-794 \gamma) q^{3}+\cdots\right) \\
q^{11 / 39}\left((14+7 \gamma)+(441+61 \gamma) q+(4445+303 \gamma) q^{2}+(30329+1167 \gamma) q^{3}+\cdots\right)
\end{array}\right)
$$

The proof for this (and for (2.6)) is similar to that sketched in Section 5.2. That there are sensible character vectors here is strong evidence for the existence of the corresponding VOA.

We see remarkable similarities between $\mathcal{D H g}$ and $\mathcal{D} S_{3}$ when $c=8$. In particular, the corresponding characters $c h_{\mathfrak{a}}, c h_{\mathfrak{c}_{i}}$ are identical, as are the sums $c h_{0}+c h_{\mathfrak{b}}$. The remaining characters $c h_{\mathfrak{d}_{1}}, \ldots, c h_{\mathfrak{d}_{6}}$ of $\mathcal{D} H g$ can have no relation with characters $c h_{\mathfrak{d}_{1}}, c h_{\mathfrak{d}_{2}}$ of $\mathcal{D} S_{3}$, since the exponents of their $q$-expansions are unrelated.

This suggests that there is a rational VOA at $c=8$, call it $\mathcal{V}_{8}$, which has conformal subalgebras realising the $\mathcal{D H g}$ and $\mathcal{D} S_{3}$ modular data (conformal subalgebra was defined at the beginning of Section 1). The $\mathcal{V}_{8}$ characters will be $c h_{0}+c h_{\mathfrak{b}}$ (the vacuum character) and some multiples of $c h_{\mathfrak{a}}, c h_{\mathfrak{c}_{i}}$, for the $c h$ 's given in (2.6).

Indeed, the knowledge of modular invariants says how this should go. We should look for modular invariants of extension type (i.e. sum of squares) for both $\mathcal{D} S_{3}$ and $\mathcal{D H g}$, which start with $\left|c h_{0}+c h_{\mathfrak{b}}\right|^{2}$. Both $\mathcal{D} S_{3}$ and $\mathcal{D} H g$ have one, namely (2.3) and (2.4). In the case of $S_{3}$ we readily identify the corresponding chiral extension: $\mathcal{V}_{8}$ corresponds to the quantum double $\mathcal{D} \mathbb{Z}_{3}$ of the cyclic group $\mathbb{Z}_{3}$. The modular invariant says two inequivalent $\mathcal{D} \mathbb{Z}_{3}$-primaries correspond to each $c h_{\mathfrak{a}}, c h_{\mathfrak{c}_{i}}$.

So $\mathcal{V}_{8}$ is the $\mathbb{Z}_{3}$-orbifold (i.e. the $\mathbb{Z}_{3}$-invariant part) of the rational $\operatorname{VOA} \mathcal{V}\left(E_{8}\right)$, for some choice of order-3 element $g$ of $E_{8}(\mathbb{R})$. The group $E_{8}(\mathbb{R})$ contains four inequivalent order 3 elements: orbifolding by any of these would give a VOA with 9 
primaries realising modular data associated to a quantum double of $\mathbb{Z}_{3}$. Half the time this double is twisted and half the time it isn't. Take one of the order 3 orbifolds corresponding to the untwisted double $\mathcal{D} \mathbb{Z}_{3}$. To recover the $\mathcal{D} S_{3}$ VOA, choose an order- 2 element $h$ of $E_{8}(\mathbb{R})$ for which $g h=h g^{-1}$ : the $h$-orbifold of $\mathcal{V}_{8}$ realises $\mathcal{D} S_{3}$. Unfortunately the Haagerup VOA won't itself be an orbifold of $\mathcal{V}_{8}$ (see Section 5.1 below), which begs Question 3 of Section 1.

To better identify $\mathcal{V}_{8}$, we should repeat the analysis for $\mathcal{D} \mathbb{Z}_{3}$. Label its 9 primaries by $(i, j) \in \mathbb{Z}_{3}^{2}$, where $(0,0)$ is the vacuum. Note that charge-conjugation $C=S^{2}$ is no longer the identity: it sends $(i, j)$ to $(-i,-j)$. Hence the characters $c h_{(i, j)}$ and $c h_{(-i,-j)}$ are equal and we should project to the subrepresentation $\operatorname{Span}\left\{c h_{(0,0)},\left(\operatorname{ch}_{(0,1)}+\operatorname{ch}_{(0,2)}\right) / 2,\left(\operatorname{ch}_{(1,0)}+\operatorname{ch}_{(2,0)}\right) / 2,\left(\operatorname{ch}_{(1,1)}+\operatorname{ch}_{(2,2)}\right) / 2,\left(\operatorname{ch}_{(1,2)}+\right.\right.$ $\left.\left.c h_{(2,1)}\right) / 2\right\}$ on which $\mathbb{Z}_{2} \cong\langle C\rangle$ acts trivially. This decomposes into $1+1+3$, where the 1 - and 3 -dimensional irreps are as before. Choosing the above basis, we obtain $\Lambda \overline{\bar{Z}}_{3}^{8}=\operatorname{diag}(2 / 3,2 / 3,2 / 3,0,1 / 3)$ and

$$
\chi_{Z 3}^{0, \equiv 8}=\left(\begin{array}{ccccc}
86 & 162 & 162 & 17496 & 2430 \\
81 & 167 & -81 & -8748 & -1215 \\
81 & -81 & 167 & -8748 & -1215 \\
3 & -3 & -3 & -12 & 18 \\
27 & -27 & -27 & 1458 & -152
\end{array}\right)
$$

It is immediate that the only character vector possible for $\mathcal{V}_{8}$ is

$$
\left(\begin{array}{c}
\operatorname{ch}_{(0,0)} \\
\operatorname{ch}_{(0,1)}=\operatorname{ch}_{(0,2)}=\operatorname{ch}_{(1,0)} \\
\operatorname{ch}_{(1,1)}=\operatorname{ch}_{(2,2)} \\
\operatorname{ch}_{(1,2)}=\operatorname{ch}_{(2,1)}
\end{array}\right)=\left(\begin{array}{c}
q^{-1 / 3}\left(1+86 q+1370 q^{2}+11586 q^{3}+\cdots\right) \\
q^{2 / 3}\left(81+1377 q+11583 q^{2}+71037 q^{3}+\cdots\right) \\
3+243 q+2916 q^{2}+21870 q^{3}+\cdots \\
q^{1 / 3}\left(27+594 q+5967 q^{2}+39852 q^{3}+\cdots\right)
\end{array}\right) .
$$

We recognise this as the character vector for the lattice $\operatorname{VOA} \mathcal{V}\left(A_{2} E_{6}\right)$, so this is the VOA $\mathcal{V}_{8}$ containing both the $S_{3}$ and Haagerup VOAs. This $\mathcal{V}_{8}$ also has, like $\mathcal{V}\left(E_{8}\right)$, an interpretation as an affine algebra VOA, and the containment $\mathcal{V}_{8} \subset \mathcal{V}\left(E_{8}\right)$ corresponds to the conformal embedding $A_{2,1} E_{6,1} \subset E_{8,1}$. It can also be realised explicitly as an orbifold - see [43. The only task remaining is to identify the Haagerup VOA as a subalgebra of $\mathcal{V}\left(A_{2} E_{6}\right)$.

\subsection{The Haagerup and $\mathrm{SO}(13)$}

The relationship with $S_{3}$ concerns the primaries $\mathfrak{a}, \mathfrak{c}_{i}$ and to a lesser extent $0, \mathfrak{b}$. There also is a striking relationship with the affine algebra modular data $B_{6,2}$ concerning the primaries $\mathfrak{d}_{l}$ (and to a lesser extent $\left.0, \mathfrak{b}\right) . \mathcal{V}\left(B_{6,2}\right)$ has central charge $c=12$, and 10 primaries we'll denote $0, \mathfrak{b}=2 \Lambda_{1}, \mathfrak{a}_{1}=\Lambda_{6}, \mathfrak{a}_{2}=\Lambda_{1}+\Lambda_{6}, \mathfrak{d}_{1}=\Lambda_{1}, \ldots, \mathfrak{d}_{5}=$ $\Lambda_{5}, \mathfrak{d}_{6}=2 \Lambda_{6}$. The $T$-matrix is $\operatorname{diag}\left(-1,-1 ;-1,1 ;-\xi_{13}^{6 l^{2}}\right)$, while the $S$-matrix is [44]

$$
S=\frac{1}{3}\left(\begin{array}{cccccccccc}
y / 2 & y / 2 & 3 / 2 & 3 / 2 & y & y & y & y & y & y \\
y / 2 & y / 2 & -3 / 2 & -3 / 2 & y & y & y & y & y & y \\
3 / 2 & -3 / 2 & 3 / 2 & -3 / 2 & 0 & 0 & 0 & 0 & 0 & 0 \\
3 / 2 & -3 / 2 & -3 / 2 & 3 / 2 & 0 & 0 & 0 & 0 & 0 & 0 \\
y & y & 0 & 0 & -c(1) & -c(2) & -c(3) & -c(4) & -c(5) & -c(6) \\
y & y & 0 & 0 & -c(2) & -c(4) & -c(6) & -c(5) & -c(3) & -c(1) \\
y & y & 0 & 0 & -c(3) & -c(6) & -c(4) & -c(1) & -c(2) & -c(5) \\
y & y & 0 & 0 & -c(4) & -c(5) & -c(1) & -c(3) & -c(6) & -c(2) \\
y & y & 0 & 0 & -c(5) & -c(3) & -c(2) & -c(6) & -c(1) & -c(4) \\
y & y & 0 & 0 & -c(6) & -c(1) & -c(5) & -c(2) & -c(4) & -c(3)
\end{array}\right)
$$


where $y=3 / \sqrt{13}$ and $c(j)=-2 y \cos (2 \pi j / 13)$ as before. Ignoring the first 4 primaries, the only difference with $\mathcal{D} H g$ are some signs.

This strongly suggests relations between $\mathcal{V}\left(B_{6,2}\right)$ and the (still hypothetical) Haagerup VOA involving the Goddard-Kent-Olive coset construction [33]. In the VOA language, the coset construction was developed in [29]; see also the lucid treatment in Section 3.11 of [47]). There are several ways this could go: e.g. the Haagerup VOA at $c=8$ could be a coset of $\mathcal{V}\left(B_{6,2}\right)$ by a $c=4$ subVOA $\mathcal{V}_{4}$. In this case the characters of $\mathcal{V}\left(B_{6,2}\right)$ (which can be determined by the Weyl-Kac character formula [42, Ch.13]) would be built from the characters of $\mathcal{V}_{4}$ and the Haagerup.

Although the possible relation between the Haagerup and $\mathcal{V}\left(B_{6,2}\right)$, in particular those involving the coset construction, seems very intriguing, for reasons of space we limit the discussion in this paper to relations between the Haagerup and $S_{3}$.

\section{Generalising the modular data of the Haagerup}

\subsection{Dihedral groups and orthogonal algebras}

Last section we observed that the Haagerup modular data is closely related to that of the symmetric group $S_{3}=\operatorname{Sym}(3)$ and affine so(13) at level 2. More generally, we propose next subsection a two-parameter generalisation of the Haagerup, related to the odd dihedral groups and affine so $(2 m+1)$ at level 2 .

First, we compute the modular data for quantum doubles of the dihedral group $D_{\nu}=\left\langle\tau, u \mid \tau^{2}, u^{\nu}, \tau u=u^{-1} \tau\right\rangle$ where $\nu=2 n+1$. Of course $S_{3} \cong D_{3}$. The twist group is $H^{3}\left(B_{D_{\nu}} ; \mathbb{T}\right)=\mathbb{Z}_{2 \nu} \cong \mathbb{Z}_{2} \times \mathbb{Z}_{\nu}$; because the Schur multipliers of $D_{\nu}$ and cyclic groups all vanish, the modular data is cohomologically trivial and is given by (1.8). The conjugacy classes of $D_{\nu}$ have representatives $e, u^{h}, \tau$ for $1 \leq h \leq n$, with centralisers $D_{\nu},\langle u\rangle,\langle\tau\rangle$ respectively. There are two 1-dimensional irreps of $D_{\nu}$ (call them $\epsilon_{0}=1, \epsilon_{1}$ ) and $n 2$-dimensional ones (call them $\sigma_{i}$ for $1 \leq i \leq n$ ); denote the $\nu$ 1-dimensional irreps of $\langle u\rangle \cong \mathbb{Z}_{\nu}$ by $\pi_{j}$ for $0 \leq j<\nu$, and the two 1-dimensional irreps of $\langle\tau\rangle \cong \mathbb{Z}_{2}$ by $\epsilon_{0}, \epsilon_{1}$ again. The primaries fall into four classes:

1. two primaries: the vacuum $0:=(e, 1)$ and $\mathfrak{b}:=\left(e, \epsilon_{1}\right)$;

2. $n$ primaries, labeled $\mathfrak{a}_{i}:=\left(e, \sigma_{i}\right)$ for $1 \leq i \leq n$;

3. $n \nu$ primaries, labeled $\mathfrak{c}_{h, j}:=\left(u^{h}, \pi_{j}\right)$ for $1 \leq h \leq n, 0 \leq j<\nu$;

4. two primaries, labeled $\mathfrak{d}_{l}:=\left(\tau, \epsilon_{l}\right), l=1,2$.

Fix a sign $s= \pm$ and integer $\omega \in \mathbb{Z}$. Corresponding to these 4 classes we have the modular data

$$
\begin{aligned}
& T=\operatorname{diag}\left(1,1 ; 1, \ldots, 1 ; \exp \left[2 \pi 1\left(\omega h^{2}+\nu h j\right) / \nu^{2}\right] ; t,-t\right), \\
& S=\frac{1}{\nu}\left(\begin{array}{cccc}
\frac{1}{2} 2 \times 2 & 1_{2 \times n} & 1_{2 \times n \nu} & \nu B \\
1_{n \times 2} & 2_{n \times n} & D & 0_{n \times 2} \\
1_{n \nu \times 2} & D^{t} & E & 0_{n \nu \times 2} \\
\nu B^{t} & 0_{2 \times n} & 0_{2 \times n \nu} & s \nu F
\end{array}\right),
\end{aligned}
$$


where $t=1,1$ for $s=1,-1$ resp., $k_{a \times b}$ for any number $k$ is the $a \times b$ matrix with constant entry $k$,

$$
B=\frac{1}{2}\left(\begin{array}{cc}
1 & 1 \\
-1 & -1
\end{array}\right) \text { and } F=\frac{1}{2}\left(\begin{array}{cc}
1 & -1 \\
-1 & 1
\end{array}\right)
$$

$D_{i,(h, j)}=2 \cos (2 \pi i h / \nu)$, and $E_{(h, j),\left(h^{\prime}, j^{\prime}\right)}=2 \cos \left(2 \pi\left(2 \omega h h^{\prime}+\nu h j^{\prime}+\nu h^{\prime} j\right) / \nu^{2}\right)$. We'll denote this modular data by $\mathcal{D}^{s, \omega} D_{\nu}$. The untwisted double is $\mathcal{D}^{+, 0} D_{\nu}$. Note that $\mathcal{D}^{s, \omega+k \nu} D_{\nu}$ is equivalent for any integer $k$, so the twist does indeed live in $\mathbb{Z}_{2 \nu}$. We've separated the order 2 from the order $\nu$ twists, for later convenience. The quantumdimensions (=statistical dimensions) $S_{* 0} / S_{00}$ of $\mathfrak{b}, \mathfrak{a}_{i}, \mathfrak{c}_{h, j}, \mathfrak{d}_{l}$ are $1,2,2, \nu$, respectively, and the global dimension $1 / S_{0,0}$ is $2 \nu$.

The $S, T$ entries live in $\mathbb{Q}\left[\xi_{4 \nu^{2}}\right]$. Choose any Galois automorphism $\sigma \in$ $\operatorname{Gal}\left(\mathbb{Q}\left[\xi_{4 \nu^{2}}\right] / \mathbb{Q}\right)$ fixing 1 and define $\ell \in \mathbb{Z}_{\nu^{2}}$ by $\sigma \xi_{\nu^{2}}=\xi_{\nu^{2}}^{\ell}$. Then applying $\sigma$ to $\mathcal{D}^{s, \omega} D_{\nu}$, as explained in Section 1.1, yields modular data equivalent to $\mathcal{D}^{s, \ell \omega} D_{\nu}$. In particular the fusion ring of $\mathcal{D}^{s, \omega} D_{\nu}$ depends only on $\nu, s$ and $\operatorname{gcd}(\omega, \nu)$. In fact, if $\ell \in \mathbb{Z}_{\nu}^{\times}$, then $\mathcal{D}^{s, \ell^{2} \omega} D_{\nu}$ and $\mathcal{D}^{s, \omega} D_{\nu}$ are equivalent.

The second ingredient going into our generalisation of the Haagerup modular data is the affine algebra modular data $B_{m, 2}$, which has central charge $c=2 m$. In particular [44], $B_{m, 2}$ has $m+4$ primaries, labelled $0, \mathfrak{b}=2 \Lambda_{1}, \mathfrak{a}_{1}=\Lambda_{m}, \mathfrak{a}_{2}=\Lambda_{1}+\Lambda_{m}$, $\mathfrak{d}_{l}=\Lambda_{l}$ for $1 \leq l<m$ and $\mathfrak{d}_{m}=2 \Lambda_{m}$. Write $\mu=2 m+1$. The $T$-matrix is $\xi_{12}^{-m} \operatorname{diag}\left(1,1 ; \xi_{8}^{m},-\xi_{8}^{m} ; \xi_{\mu}^{m l^{2}}\right)$, while the $S$-matrix is

$$
S=\left(\begin{array}{ccc}
\frac{x}{2} 2 \times 2 & B & x_{2 \times m} \\
B^{T} & F & 0_{2 \times m} \\
x_{m \times 2} & 0_{m \times 2} & H
\end{array}\right)
$$

where $x=1 / \sqrt{\mu}, B$ and $F$ are as above, and $H_{l, l^{\prime}}=2 x \cos \left(2 \pi l l^{\prime} / \mu\right)$. The quantumdimensions of $\mathfrak{b}, \mathfrak{a}_{i}, \mathfrak{d}_{l}$ are $1, \sqrt{\mu}, 2$, respectively, with global dimension $2 \sqrt{\mu}$.

There is no obvious twist of the $B_{m, 2}$. Curiously [15], affine so( $\left.\nu^{2}\right)$ at level 2 coincides with $\mathcal{D}^{\omega} D_{\nu}$ for a specifically chosen twist $\omega$ (this is one of the few overlaps of affine algebra and finite group modular data [15]), but that fact seems to have no role in our story (in particular we are not interested in the values $m=\left(\nu^{2}-1\right) / 2$ ).

\subsection{Generalising the Haagerup modular data}

Sections 2.3, 2.5 and 3.1 suggest a generalisation of $\mathcal{D H g}$. Write $\nu=2 n+1$ for $n \geq 0$, and choose $\omega \in \mathbb{Z}$ as before. Write $m=2\left(n^{2}+n+1\right), \mu=2 m+1=\nu^{2}+4$, and $\delta=(\nu+\sqrt{\mu}) / 2$. Note that $\delta$ satisfies $\delta^{2}=\nu \delta+1$ and lies in the range $\nu<\delta<\nu+\frac{1}{2}$. Again the primaries fall into four classes:

1. two primaries, denoted 0 and $\mathfrak{b}$;

2. $n$ primaries, denoted $\mathfrak{a}_{i}$ for $1 \leq i \leq n$;

3. $n \nu$ primaries, denoted $\mathfrak{c}_{h, j}$ for $1 \leq h \leq n, 0 \leq j<\nu$;

4. $m$ primaries, denoted $\mathfrak{d}_{l}$ for $1 \leq l \leq m$. 
Breaking $S$ and $T$ into 16 blocks, as in the previous subsection, we get

$$
\begin{aligned}
T & =\operatorname{diag}\left(1,1 ; 1, \ldots, 1 ; \exp \left[2 \pi 1\left(\omega h^{2}+\nu h i\right) / \nu^{2}\right] ; \exp \left[2 \pi 1 l^{2} m / \mu\right]\right), \\
S & =\frac{1}{\nu}\left(\begin{array}{cccc}
A & 1_{2 \times n} & 1_{2 \times n \nu} & B^{\prime} \\
1_{n \times 2} & 2_{n \times n} & D & 0_{n \times m} \\
1_{n \nu \times 2} & D^{t} & E & 0_{n \nu \times m} \\
B^{\prime t} & 0_{m \times n} & 0_{m \times n \nu} & -\nu H
\end{array}\right),
\end{aligned}
$$

where $k_{a \times b}, D, E$ and $H$ are as in Section $3.1\left(\right.$ so $-\nu H_{l, l^{\prime}}=-2 y \cos \left(2 \pi l l^{\prime} / \mu\right)$ ), and

$$
A=\frac{1}{2}\left(\begin{array}{ll}
1-y & 1+y \\
1+y & 1-y
\end{array}\right) \text { and } B^{\prime}=y\left(\begin{array}{cccc}
1 & 1 & \cdots & 1 \\
-1 & -1 & \cdots & -1
\end{array}\right)
$$

for $y=\frac{\nu}{\sqrt{\mu}}$. Denote this modular data by $\mathcal{D}^{\omega} \mathrm{Hg}_{2 n+1}$; we call $\mathcal{D}^{0} \mathrm{Hg}_{\nu}$ HaagerupIzumi modular data. To our knowledge this modular data is new except when $n \leq 1$ and $\nu$ divides $\omega$; in particular twisting the Haagerup data was unanticipated in the literature. We discuss further generalisations next subsection.

The quantum dimensions (or statistical dimensions) $S_{*, 0} / S_{0,0}$ for $\mathfrak{b}, \mathfrak{a}_{i}, \mathfrak{c}_{h, j}, \mathfrak{d}_{l}$ are respectively $1+\nu \delta, 2+\nu \delta, 2+\nu \delta$, and $\nu \delta$. The global dimension is $1 / S_{0,0}=\nu(\nu \delta+2)$. Note that the submatrix $-\nu H$ has exactly half of the $\mu-1$ rows and columns of $S$.

The fusions can be computed directly using Verlinde's formula (1.1) (or from Proposition 2 below). As mentioned in Section 1.1, it suffices to consider all $i, j, k$ different from 0 . For $\mathcal{D}^{\omega} \mathrm{Hg}_{\nu}$, all of those fusion coefficients are 1 except for

$$
\begin{aligned}
& N_{\mathfrak{b}, \mathfrak{a}_{i}, \mathfrak{a}_{i}}=2, N_{\mathfrak{b}, \mathfrak{c}_{h, i}, \mathfrak{c}_{h, i}}=2, N_{\mathfrak{b}, \mathfrak{d}_{l}, \mathfrak{d}_{l}}=0, N_{\mathfrak{a}_{i}, \mathfrak{a}_{j}, \mathfrak{a}_{k}}=2 \text { if } k \equiv_{\nu} s i+s^{\prime} j, \\
& N_{\mathfrak{c}_{h, i}, \mathfrak{c}_{h^{\prime}, j}, \mathfrak{c}_{h^{\prime \prime}, k}}=2 \text { if } h^{\prime \prime} \equiv_{\nu} s h+s^{\prime} h^{\prime}, k \equiv_{\nu} s i+s^{\prime} j+2 \omega\left(s h+s^{\prime} h^{\prime}-h\right) / \nu, \\
& N_{\mathfrak{a}_{i}, \mathfrak{c}_{h, j}, \mathfrak{c}_{h, k}}=2 \text { if } s i \equiv_{\nu} j-k, N_{\mathfrak{d}_{l}, \mathfrak{d}_{l^{\prime}}, \mathfrak{d}_{l^{\prime \prime}}}=0 \text { if } l^{\prime \prime} \equiv_{\mu} s l+s^{\prime} l^{\prime},
\end{aligned}
$$

where $s, s^{\prime} \in\{ \pm 1\}$ are arbitrary signs.

As in Section 3.1, $\mathcal{D}^{\omega} \mathrm{Hg}_{2 n+1}$ depends up to equivalence on the value of the twist $\omega \bmod \nu$; the fusion ring depends up to equivalence only on $n$ and $\operatorname{gcd}(\nu, \omega)$; and $\mathcal{D}^{\omega} \mathrm{Hg}_{2 n+1} \cong \mathcal{D}^{\ell^{2} \omega} \mathrm{Hg}_{2 n+1}$ for any $\ell$ coprime to $\nu$.

The computation of fusions reduces to the identity

$$
8 \sum_{d=1}^{k} \cos \left(\frac{2 \pi a d}{2 k+1}\right) \cos \left(\frac{2 \pi b d}{2 k+1}\right) \cos \left(\frac{2 \pi c d}{2 k+1}\right)=-4+(2 k+1) s
$$

proved using $2 \cos (x)=e^{1 x}+e^{-1 x}$, where $0 \leq s \leq 4$ is the number of pairs $\left(s_{a}, s_{b}\right)$ of signs \pm 1 such that $c \equiv_{2 k+1} s_{a} a+s_{b} b$. Modularity reduces to the Gauss sum

$$
\sum_{a=0}^{2 k} \exp \left[2 \pi \mathrm{r} c a^{2} /(2 k+1)\right]=\sqrt{2 k+1}\left(\frac{c}{2 k+1}\right)\left\{\begin{array}{ll}
1 & \text { for } k \text { even } \\
1 & \text { for } k \text { odd }
\end{array} .\right.
$$

Since $\mu \equiv_{8} 5, \mu$ can never be a perfect square, and the Jacobi symbol $\left(\frac{m}{\mu}\right)$ will always equal -1 . See Proposition 2 below for a more elegant argument. 
Of course $\mathcal{D}^{0} \mathrm{Hg}_{3}$ recovers the original Haagerup double $\mathcal{D} H g$, given in Section 2.3. The similarity with the modular data $\mathcal{D}^{+, \omega} D_{\nu}$ and $B_{m, 2}$ is evident (ignoring the first 4 primaries of $B_{m, 2}$, the only difference with $\mathcal{D}^{\omega} \mathrm{Hg}_{\nu}$ is that the bottom-right corner of both $T$ and $S$ are off by some 6 th root of 1 ). In particular, class 1 runs through the 2-dimensional irreps of $D_{\nu}$, while $h$ and $j$ in class 3 parametrise the size-2 conjugacy classes in $D_{\nu}$ and the irreps of $\mathbb{Z}_{\nu}$, respectively; class 4 runs through the fundamental nonspinorial weights of $\mathrm{so}(\mu)$.

The mysterious ' 13 ' in $\mathcal{D} H g$ is thus $4+3^{2}$, where the 3 here references the normal subgroup $\mathbb{Z}_{3}$ of $S_{3}$. It is tempting to guess that the 4 is $2^{2}$, where the ' 2 ' is the involution of $S_{3}$. This suggests the further generalisation of $\mathcal{D}^{\omega} \mathrm{Hg}_{\nu}$ given in the Wildly Optimistic Guess of Section 1.

\subsection{Further generalisations}

The way in which $\mathcal{D}^{\omega} \mathrm{Hg}_{\nu}$ is built from $\mathcal{D}^{+, \omega} D_{\nu}$ and $B_{m, 2}$ leads to the notion of grafting modular data. A simple instance is provided by the following proposition, but it can be massively generalised. For instance it would be interesting to extend it to vacuum blocks larger than $2 \times 2$.

For simplicity restrict here to unitary modular data (recall Section 1.1) with real matrix $S$. Call modular data $(\Phi, 0, S, T) \mathbb{Z}_{2}$-laminated if it can be written in block form $\Phi=\left(0, \mathfrak{b} ; \mathfrak{a}_{1}, \ldots, \mathfrak{a}_{m} ; \mathfrak{d}_{1}, \ldots, \mathfrak{d}_{n}\right)$ such that $S_{0, \mathfrak{b}}=S_{0,0}, N_{\mathfrak{b}, \mathfrak{a}_{i}}^{\mathfrak{a}_{i}} \neq 0, T_{\mathfrak{b}, \mathfrak{b}}=T_{0,0}$ and $S_{\mathfrak{b}, \mathfrak{d}_{j}}<0$. It is elementary to verify that modular data is $\mathbb{Z}_{2}$-laminated iff it can be written in the form

$$
S=\left(\begin{array}{cccc}
x & x & \vec{a} & \vec{d} \\
x & x & \vec{a} & -\vec{d} \\
\vec{a}^{T} & \vec{a}^{T} & A & 0_{m \times n} \\
\vec{d}^{T} & -\vec{d}^{T} & 0_{m \times n} & D
\end{array}\right), \quad T=\operatorname{diag}(r, r ; \vec{s} ; \vec{t}),
$$

for some numbers $x, r \in \mathbb{R}$, row vectors $\vec{a}, \vec{s} \in \mathbb{R}^{m}, \vec{d}, \vec{t} \in \mathbb{R}^{n}$ and matrices $A, D$. The condition $S_{\mathfrak{b}, 0}=S_{0,0}$ implies the matrix $N_{\mathfrak{b}}$ with entries $N_{\mathfrak{b}, i}^{j}$ for all $i, j \in \Phi$ is a permutation matrix (see e.g. [31]); in conformal field theory, such a primary $\mathfrak{b}$ is called a simple-current. The modular data $\mathcal{D}^{ \pm, \omega} D_{\nu}$ and $B_{r, 2}$ are both $\mathbb{Z}_{2}$-laminated, for any odd $\nu$ and any twist $( \pm, \omega)$ and any rank $r$, as is $A_{1,4}$.

Proposition 2. Consider $\mathbb{Z}_{2}$-laminated modular data $(\Phi, 0, S, T)$ and $\left(\Phi^{\prime}, 0^{\prime}, S^{\prime}, T^{\prime}\right)$.

(a) There are integers $M>0, L \geq 0, N>0$ such that $2 x=1 / \sqrt{M}, N$ and $4^{L}$ divide $4 M, 2^{1-L} \sqrt{M} \vec{a} \in \mathbb{Z}^{m}, 2 \sqrt{M / N} \vec{d} \in \mathbb{Z}^{n}$, and the gcd over all components $2^{1-L} \sqrt{M} a_{i}$ is 1 (likewise for the components $2 \sqrt{M / N} d_{k}$ ).

(b) Suppose $x=x^{\prime}$ (that is, the global dimensions agree) and $r=r^{\prime}$ (i.e. the central 
charges $c, c^{\prime}$ agree mod 24). Then the following defines modular data:

$$
\widehat{S}=\left(\begin{array}{cccc}
x & x & \vec{a} & \vec{d}^{\prime} \\
x & x & \vec{a} & -\vec{d}^{\prime} \\
\vec{a}^{T} & \vec{a}^{T} & A & 0 \\
\vec{d}^{T} & -\vec{d}^{T} & 0 & D^{\prime}
\end{array}\right), \widehat{T}=\operatorname{diag}\left(r, r ; \vec{s} ; \vec{t}^{\prime}\right) .
$$

(c) Suppose $x>x^{\prime}$ and $r=-r^{\prime} \omega$ for some 3rd root $\omega$ of 1 (that is, $c-c^{\prime} \equiv_{8} 4$ ). Define $x_{ \pm}=x \pm x^{\prime}$. Then

$$
\widehat{S}=\left(\begin{array}{cccc}
x_{-} & x_{+} & \vec{a} & \vec{a}^{\prime} \\
x_{+} & x_{-} & \vec{a} & -\vec{a}^{\prime} \\
\vec{a}^{T} & \vec{a}^{T} & A & 0 \\
\vec{a}^{T} & -\vec{a}^{T} & 0 & -A^{\prime}
\end{array}\right), \widehat{T}=\operatorname{diag}\left(r, r ; \vec{s} ;-\omega \vec{t}^{\prime}\right)
$$

satisfies all conditions of modular data except possibly that the fusion coefficients are nonnegative integers. Its fusion coefficients are all integers iff $4 \mid\left(M^{\prime}-M\right)$, and both

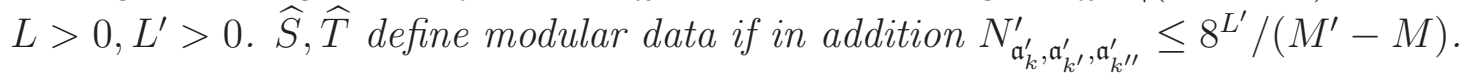

Proof. Recall (1.4). Clearly, any $\sigma$ permutes $\{0, \mathfrak{b}\}$, so the number $x^{-2}$ will be fixed by any $\sigma$. But $x^{-2}$ is an algebraic integer, being the sum of squares of certain eigenvalues $S_{i, 0} / S_{0,0}$ of integer matrices. Therefore $x^{-2}$ is indeed an integer. Computing $N_{\mathfrak{a}_{i}, \mathfrak{d}_{k}, \mathfrak{o}_{k}} / N_{\mathfrak{a}_{j}, \mathfrak{d}_{k}, \mathfrak{d}_{k}}=a_{i} / a_{j}$ and $N_{\mathfrak{a}_{i}, \mathfrak{d}_{k}, \mathfrak{d}_{k^{\prime}}} / N_{\mathfrak{a}_{i}, \mathfrak{d}_{k}, \mathfrak{d}_{k}}=d_{k^{\prime}} / d_{k}$, we see that there are $\beta, \gamma>0$ for which both vectors $\beta \vec{a}$ and $\gamma \vec{d}$ are integral. The gcd condition follows by choosing these $\beta, \gamma$ as small as possible. Because $\vec{d} \cdot \vec{d}=1 / 2$, we know $\gamma^{2} \in \mathbb{Q}$. Because $N_{\mathfrak{a}_{i}, \mathfrak{d}_{k}, \mathfrak{d}_{k}}=2 a_{i} d_{k}^{2} / x \in \mathbb{Z}$ and each quantum-dimension $d_{k} / x$ is an algebraic integer, we know $a_{i} / x \in \mathbb{Z}$ and hence $(\beta x)^{-1} \in \mathbb{Z}$. From $2 x^{2}+\vec{a} \cdot \vec{a}=1 / 2$ we now see that 4 divides $x^{-2}$, and $(\beta x)^{-1}$ is a power $2^{L}$ of 2 . This gives us (a).

The proof of (b) and (c) is now straightforward. Most interesting are the fusion calculations in (c). Note that $4 x^{2} x^{\prime 2} /\left(x^{2}-x^{\prime 2}\right)=1 /\left(M^{\prime}-M\right), a_{i} / x \in 2^{L} \mathbb{Z}$ and $a_{k}^{\prime} / x^{\prime} \in 2^{L^{\prime}} \mathbb{Z}$. We obtain

$$
\begin{gathered}
\widehat{N}_{\mathfrak{b}, \mathfrak{b}, \mathfrak{b}}=\frac{4}{M^{\prime}-M}, \widehat{N}_{\mathfrak{b}, \mathfrak{b}, \mathfrak{a}_{i}}=\frac{2 a_{i}}{x\left(M^{\prime}-M\right)}, \widehat{N}_{\mathfrak{b}, \mathfrak{a}_{i}, \mathfrak{a}_{i^{\prime}}}=\frac{a_{i} a_{i^{\prime}}}{x^{2}\left(M^{\prime}-M\right)}+\delta_{i, i^{\prime}}, \\
\widehat{N}_{\mathfrak{b}, \mathfrak{b}, \mathfrak{a}_{k}^{\prime}}=\frac{2 a_{k}^{\prime}}{x^{\prime}\left(M^{\prime}-M\right)}, \widehat{N}_{\mathfrak{b}, \mathfrak{a}_{i}, \mathfrak{a}_{k}^{\prime}}=\frac{a_{i} a_{k}^{\prime}}{x x^{\prime}\left(M^{\prime}-M\right)}, \widehat{N}_{\mathfrak{b}, \mathfrak{a}_{k}^{\prime}, \mathfrak{a}_{k^{\prime}}^{\prime}}=\frac{a_{k}^{\prime} a_{k^{\prime}}^{\prime}}{x^{\prime 2}\left(M^{\prime}-M\right)}-\delta_{k, k^{\prime}}, \\
\widehat{N}_{\mathfrak{a}_{i}, \mathfrak{a}_{i^{\prime}}, \mathfrak{a}_{i^{\prime \prime}}}=\frac{a_{i} a_{i^{\prime}} a_{i^{\prime \prime}}}{2 x^{3}\left(M^{\prime}-M\right)}+N_{\mathfrak{a}_{i}, \mathfrak{a}_{i^{\prime}}, \mathfrak{a}_{i^{\prime \prime}}}, \widehat{N}_{\mathfrak{a}_{i}, \mathfrak{a}_{i^{\prime}}, \mathfrak{a}_{k}^{\prime}}=\frac{a_{i} a_{i^{\prime}} a_{k}^{\prime}}{2 x^{2} x^{\prime}\left(M^{\prime}-M\right)}, \\
\widehat{N}_{\mathfrak{a}_{i}, \mathfrak{a}_{k}^{\prime}, \mathfrak{a}_{k^{\prime}}^{\prime}}=\frac{a_{i} a_{k}^{\prime} a_{k^{\prime}}^{\prime}}{2 x x^{\prime 2}\left(M^{\prime}-M\right)}, \widehat{N}_{\mathfrak{a}_{k}^{\prime}, \mathfrak{a}_{k^{\prime}}^{\prime}, \mathfrak{a}_{k^{\prime \prime}}^{\prime}}=\frac{a_{k}^{\prime} a_{k^{\prime}}^{\prime} a_{k^{\prime \prime}}^{\prime}}{2 x^{\prime 3}\left(M^{\prime}-M\right)}-N_{\mathfrak{a}_{k}^{\prime}, \mathfrak{a}_{k^{\prime}}^{\prime}, \mathfrak{a}_{k^{\prime \prime}}^{\prime}} .
\end{gathered}
$$

QED

We call $\widehat{S}, \widehat{T}$ the graft of $S^{\prime}, T^{\prime}$ onto $S, T$. Of course $\mathcal{D}^{\omega} \mathrm{Hg}_{\nu}$ is the graft of $B_{m, 2}$ onto $\mathcal{D}^{ \pm, \omega} D_{\nu}$; here $M^{\prime}=\mu, M=\nu^{2}$ (so $M^{\prime}-M=4$ ), $\omega=\xi_{3}^{n^{2}+n-2}$, and $L=L^{\prime}=2$. An easy example of (b) is grafting $\mathcal{D}^{-, 0} D_{\nu}$ onto $\mathcal{D}^{+, \omega} D_{\nu}$ to form $\mathcal{D}^{-, \omega} D_{\nu}$. 
An easy generalisation of $\mathcal{D}^{\omega} \mathrm{Hg}_{\nu}$ is to replace $\mathbb{Z}_{\nu}$ with any abelian group $K$ of odd order $\nu$. Take a trivial twist $\omega=0$, for simplicity. The entries of $S$ and $T$ involving classes 1 and 4 are identical to those in (3.3). The role of $D_{\nu}$ is played by the semidirect product $D_{K}=K \rtimes \mathbb{Z}_{2}$, so the class 2 primaries are labelled by the 2-dimensional irreps $i$ of $D_{K}$, and the class 3 ones by pairs $(h, j)$ where $h$ runs through representatives of the cardinality-2 conjugacy classes of $D_{K}$ and $j$ runs through the irreps of $K$. The T-entries for class 2 are again 1's, while those for class 3 are the evaluation $j(h)$. The submatrices $D$ and $E$ are again cosines, namely $D_{i,(h, j)}=i(h)$ and $E_{(h, j),\left(h^{\prime}, j^{\prime}\right)}=$ $2 \operatorname{Re}\left(j^{\prime}(h) j\left(h^{\prime}\right)\right)$. Although the modular data thus generalises naturally to arbitrary odd order abelian groups, we see in Section 4.1 that the subfactor realisation for noncyclic $K$ is more subtle.

It is tempting to look for additional twists of $\mathcal{D H g}_{\nu}$. After all, we've suggested that the generalised Haagerup is a twin of the dihedral $D_{\nu}$, and the latter can be twisted by $\mathbb{Z}_{2 \nu}$ and not merely $\mathbb{Z}_{\nu}$. The $\mathbb{Z}_{\nu}$ twist of both $\mathcal{D} D_{\nu}$ and $\mathcal{D} \mathrm{Hg}_{\nu}$ affects the class 3 primaries. The independent $\mathbb{Z}_{2}$ twist of $\mathcal{D} D_{\nu}$ affects the class 4 primaries, so this suggests one should look for additional twists (by $\mathbb{Z}_{2}$ or perhaps $\mathbb{Z}_{\mu}$ ) of $\mathcal{D} H g_{\nu}$, which sees the $m$ primaries of class 4 and leaves the other classes untouched.

We haven't gotten this to work. For instance, one approach to probing some of the missing twists would be a Galois automorphism acting on $S$ and $T$ entry-by-entry. The entries of $\mathcal{D}^{\omega} \mathrm{Hg}_{\nu}$ lie in $\mathbb{Q}\left[\xi_{\nu^{2} \mu}\right]$, with Galois group $\mathbb{Z}_{\nu^{2}}^{\times} \times \mathbb{Z}_{\mu}^{\times}$. The class 3 twists $\omega \in \mathbb{Z}_{\nu}$ are stable under $\mathbb{Z}_{\nu^{2}}^{\times}$, as explained last subsection, so we should consider the effect of $\mathbb{Z}_{\mu}^{\times}$on $\mathcal{D}^{\omega} \mathrm{Hg}_{\nu}$.

For $\ell \in \mathbb{Z}_{\mu}^{\times}, \sigma_{\ell} y= \pm y$ for some sign. If $\sigma_{\ell} y=-y$, then $\sigma_{\ell}$ sends $\mathcal{D}^{\omega} \mathrm{Hg}_{\nu}$ to nonunitary modular data with exactly the same fusion coefficients: the vacuum primary is still the first one, but the positive column of $S$ is the second one. This means this modular data won't have a subfactor realisation (it could perhaps have a planar algebra interpretation, if the requirement of positive-definiteness there is dropped), though it can still have a VOA one. The notion of twist should presumably preserve unitarity, so we won't regard this $\sigma_{\ell} \mathcal{D}^{\omega} \mathrm{Hg}_{\nu}$ as a twist. Nevertheless it may be interesting to search for its VOA realisations.

For this reason we should focus on those $\ell \in \mathbb{Z}_{\mu}^{\times}$for which $\sigma_{\ell} y=+y$. Now, it is typical for small $n$ that $\mu$ is a prime power (this is true for all $\nu<19$ except $\nu=9$ ). In this case, any such $\sigma_{\ell}$ will send $\mathcal{D}^{\omega} \mathrm{Hg}_{\nu}$ to equivalent modular data. The search for new twists using Galois seems to fail.

However when $\mu$ is not a prime power, this idea bears fruit. Given the prime decomposition $\mu=\prod_{i=1}^{k} p_{i}^{m_{i}}$, where each $m_{i}>0$, then $\sigma_{\ell} \in \operatorname{Gal}\left(\mathbb{Q}\left[\xi_{\nu^{2} \mu}\right] / \mathbb{Q}\right) \cong \mathbb{Z}_{\nu^{2} \mu}^{\times}$ fixes $\delta$ iff $\prod_{i=1}^{k}\left(\frac{\ell}{p_{i}}\right)=+1$. We can also require here that $\ell \equiv_{\nu^{2}} 1$ (the $\nu^{2}$-part of $\ell$ merely shuffles the twists $\omega$ ). The automorphism $\sigma_{\ell}$ maps $\mathcal{D}^{\omega} \mathrm{Hg}_{\nu}$ to equivalent modular data iff $\left(\frac{\ell}{p_{i}}\right)=+1 \forall i$. Given any set $P$ (possibly empty) containing an even number of distinct prime divisors of $\mu$, pick any $\ell_{P} \in \mathbb{Z}_{\nu^{2} \mu}^{\times}$with $\ell_{P} \equiv{ }_{\nu^{2}} 1$ and $\left(\frac{\ell}{p_{i}}\right)=-1$ iff $p_{i} \in P$. By $\mathcal{D}^{\omega, P} \mathrm{Hg}_{\nu}$ we mean the modular data $\sigma_{\ell_{P}} S, \sigma_{\ell_{P}} T$; up to equivalence it is well-defined, and is inequivalent to $\mathcal{D}^{\omega} \mathrm{Hg}_{\nu}$ even though it has the 
same fusions. Thus as long as $k>1$, i.e. $\mu$ is not a prime power, this construction yields new modular data. In Section 4.1 we find subfactor realisations for this new Galois twist, at least when $\nu=9$ and $\nu=19$.

We learned in Section 2.4 that $\mathcal{D H g}$ also sees $\mathcal{D} \mathbb{Z}_{3}$, in some ways more directly than it does $\mathcal{D} S_{3}$. Since $\mathcal{D} \mathbb{Z}_{\nu}$ has only a $\mathbb{Z}_{\nu}$ worth of twists, it is certainly not inconceivable that the correct generic twist group for $\mathcal{D H g}$ is indeed $\mathbb{Z}_{\nu}$.

\subsection{Miscellanea involving modular invariants}

[26, Sect. 6] studied the quantum double $S_{3}$ modular data in detail, and similar techniques [28] can be used for their twists, as noted in [21]. Using [27, Theorem 4.3],28] (with the correction to [27] where $Z_{11}$ was erroneously recorded as being

sufferable, when we have now seen in Section 2.2 of this paper that it is indeed a dual canonical modular invariant), we can summarize these results in the following table (nimless means there is no compatible nimrep, while nimble means there is; sufferable means there is a subfactor realisation):

\begin{tabular}{|l||c|c|c|c|}
\hline & nimless & nimble, insufferable & sufferable & total \\
\hline $\mathcal{D}^{+, 0} S_{3}$ & 14 & 6 & 28 & 48 \\
\hline $\mathcal{D}^{-, 1} S_{3}$ & 1 & 0 & 4 & 5 \\
\hline $\mathcal{D}^{+, 1} S_{3}$ & 1 & 0 & 8 & 9 \\
\hline $\mathcal{D}^{-, 0} S_{3}$ & 9 & 7 & 12 & 28 \\
\hline $\mathcal{D}^{0} \mathrm{Hg}_{3}$ & $?$ & $?$ & $8 \leq ? \leq 13$ & 28 \\
\hline
\end{tabular}

Table 1. Modular invariants for the doubles of $\operatorname{Sym}(3)$ and Haagerup

The commutant of $S, T$ of course forms an algebra under matrix multiplication, but the sufferable modular invariants themselves have a fusion structure, in the sense that the product of two of them is always a linear combination over nonnegative integers of other sufferable modular invariants. The modular invariants for $\mathcal{D}^{s, \omega} D_{\nu}$ and $\mathcal{D}^{s, \ell \omega} D_{\nu}$, including this fusion structure, are naturally isomorphic for any $\ell \in \mathbb{Z}_{\nu}^{\times}$, using $\sigma_{\ell} \mathcal{D}^{s, \omega} D_{\nu} \cong \mathcal{D}^{s, \ell \omega} D_{\nu}$; the same applies to $\mathcal{D}^{\omega} \mathrm{Hg}_{\nu}$ and $\mathcal{D}^{\ell \omega} \mathrm{Hg}_{\nu}$.

[28, 21] remarked that there is an (injective) homomorphism from the $28 \mathrm{DHg}$ modular invariants to the 28 sufferable $\mathcal{D}^{+, 0} S_{3}$ ones. We can define a natural injective homomorphism $\phi_{\left(S_{3}, s \omega\right)}^{\left(S_{3},+0\right)}$ from the sufferable twist $s, \omega$ quantum double $S_{3}$ modular invariants into the untwisted $S_{3}$ ones. We can actually embed the sufferable -1 into +1 modular invariants, the sufferable -1 into the -0 modular invariants and the sufferable -0 into +0 modular invariants.

This can be generalised and understood as follows. As is clear from Sections 3.1 and 3.2, primaries for both $\mathcal{D}^{s, \omega} D_{\nu}$ and $\mathcal{D}^{\omega} \mathrm{Hg}_{\nu}$ fall naturally into 4 classes, and so their modular invariants decompose naturally into $4 \times 4$ blocks as did their $S, T$ matrices. Write $S_{[i],[j]}, T_{[i],[i]}, M_{[i],[j]}$ for these blocks. 
Theorem 3. Write $k$ for the number of distinct prime divisors of $\mu$. Define a map

$$
{ }^{\omega} \phi_{\mathrm{Hg} \nu}^{D \nu} M=\left(\begin{array}{cccc}
M_{[1],[1]} & M_{[1],[2]} & M_{[1],[3]} & 0_{2 \times 2} \\
M_{[2],[1]} & M_{[2],[2]} & M_{[2],[3} & 0_{n \times 2} \\
M_{[3],[1]} & M_{[3],[2]} & M_{[3],[3]} & 0_{n \nu \times 2} \\
0_{2 \times 2} & 0_{2 \times n} & 0_{2 \times n \nu} & (a-b) I_{2 \times 2}
\end{array}\right)
$$

where $M_{[1],[1]}=\left(\begin{array}{ll}a & b \\ c & d\end{array}\right)$. Then ${ }^{\omega} \phi_{\mathrm{Hg} \nu}^{D \nu}$ is a bijection between all modular invariants of $\mathcal{D}^{\omega} \mathrm{Hg}_{\nu}$ with $b \neq 0$, and all modular invariants of $\mathcal{D}^{-, \omega} D_{\nu}$ with $b \neq 0$. ${ }^{\omega} \phi_{\mathrm{Hg} \nu}^{D \nu}$ is a $2^{k-1}$-to-1 surjection from all modular invariants of $\mathcal{D}^{\omega} \mathrm{Hg}_{\nu}$ with $b=0$, onto all modular invariants of $\mathcal{D}^{-, \omega} D_{\nu}$ with $b=0$. Moreover, any modular invariant of $\mathcal{D}^{-, \omega} D_{\nu}$ is a modular invariant of $\mathcal{D}^{+, \omega} D_{\nu}$ (but not conversely).

Proof. Let $M$ and $M^{\prime}$ be modular invariants for $\mathcal{D}^{\omega} \mathrm{Hg}_{\nu}$ and $\mathcal{D}^{-, \omega} D_{\nu}$, respectively. Let $S, T$ (resp. $S^{\prime}, T^{\prime}$ ) denote the modular data for $\mathcal{D}^{\omega} \mathrm{Hg}_{\nu}$ (resp. $\mathcal{D}^{-, \omega} D_{\nu}$ ). From $M^{\prime} T^{\prime}=T^{\prime} M^{\prime}$ we see that $M_{[i],[4]}^{\prime}=0=M_{[4],[i]}^{\prime}$ for $i=1,2,3$. We also see that $M_{[4],[4]}^{\prime}$ is diagonal. Recall (1.12): we see from the 0 -row of $S$ that $s_{\ell}$ in classes [1],[2],[3] is identically +1 for all $\ell \in \mathbb{Z}_{\nu^{2} \mu}^{\times}$, while $s_{\ell}\left(\mathfrak{d}_{l}\right)=\left(\frac{\ell}{\mu}\right)$ equals -1 when e.g. $\ell \equiv_{\mu} m$. Thus also $M_{[i],[4]}=0=M_{[4],[i]}$ for $i=1,2,3$.

Evaluating $M S=S M$ at ([1], [4]) and ([4], [1]), we get two possibilities (identical conclusions hold for $\left.M^{\prime}\right)$ :

(i) $M_{[1],[1]}=I$ and $M_{[4],[4]}$ is a permutation matrix: $M_{\mathfrak{v}_{l}, \mathfrak{o}^{\prime}}=\delta_{\pi l, l^{\prime}}$ for some permutation $\pi$ of $1 \leq l \leq m$;

(ii) $M_{[1],[1]}=1_{2 \times 2}$ and $M_{[4],[4]}=0$.

Looking at the remaining equations $M S=S M, M T=T M, M^{\prime} S^{\prime}=S^{\prime} M^{\prime}$ and $M^{\prime} T^{\prime}=T^{\prime} M^{\prime}$, we see that (in case (i)) the equations involving $M_{[4],[4]}$ decouple from the others and reduce to

$$
\pi(l) \pi\left(l^{\prime}\right) \equiv_{\mu} \pm l l^{\prime}, \pi(l)^{2} \equiv_{\mu} l^{2} .
$$

On the other hand, the equations for the unknown entries of $M_{[i],[j]}, i, j \neq 4$, are identical to the corresponding equations for $M_{[i],[j]}^{\prime}$. For example, $(M S)_{[2],[4]}=(S M)_{[2],[4]}$ and $\left(M^{\prime} S^{\prime}\right)_{[2],[4]}=\left(S^{\prime} M^{\prime}\right)_{[2],[4]}$ both reduce to $M_{\mathfrak{a}_{i}, 0}=M_{\mathfrak{a}_{i}, \mathfrak{b}}$ and $M_{\mathfrak{a}_{i}, 0}^{\prime}=M_{\mathfrak{a}_{i}, \mathfrak{b}}^{\prime}$. Therefore in case (ii), where $M_{[4],[4]}=0=M_{[4],[4]}^{\prime}$, this means ${ }^{\omega} \phi_{\mathrm{Hg} \nu}^{D \nu}$ is indeed a bijection.

In case (i), we know $M_{[4],[4]}^{\prime}=I$, so we need to identify the permutation $\pi$. Write $\pi 1=\ell$. Then (3.5) requires $\ell^{2} \equiv_{\mu} 1$, hence $\pi l \equiv_{\mu} \pm l \ell$. So to any $1 \leq \ell \leq m$ satisfying $\ell^{2} \equiv_{\mu} 1$, define $\pi_{\ell} l$ to be the unique number $1 \leq \pi_{\ell} l \leq m$ obeying $\pi_{\ell} l \equiv_{\mu} \pm l \ell$. Then $\pi_{\ell}$ satisfies (3.5) and thus defines the $M_{[4],[4]}$-block of a case (i) $\mathcal{D}^{\omega} \mathrm{Hg}_{\nu}$ modular invariant. These $2^{k-1} \ell$ parametrise the kernel of ${ }^{\omega} \phi_{\mathrm{Hg} \nu}^{D \nu}$ in case (i).

$\mathcal{D}^{+, \omega} D_{\nu}$ is handled identically. QED

Note that ${ }^{\omega} \phi_{\mathrm{Hg} \nu}^{D \nu}$ is linear and preserves matrix multiplication. In particular, when $\mu$ is a prime-power (as it is for the original Haagerup $\mathcal{D H g}$, and all $\nu<19$ except $\nu=$ 
$9)$, there will be an algebra isomorphism between the span of modular invariants for $\mathcal{D}^{\omega} \mathrm{Hg}_{\nu}$ and for $\mathcal{D}^{-, \omega} D_{\nu}$, and an (injective but nonsurjective) algebra homomorphism of these into $\mathcal{D}^{+, \omega} D_{\nu}$.

The relation between the 28 sufferable $\mathcal{D}^{+, 0} S_{3}$ modular invariants and the 28 $\mathcal{D}^{0} \mathrm{Hg}_{3}$ modular invariants would seem to be a coincidence: e.g. at $\nu=1, \mathcal{D}^{+, 0} D_{1}=$ $\mathcal{D}^{+, 0} \mathbb{Z}_{2}$ has exactly 6 modular invariants, all sufferable, while $\mathcal{D}^{0} \mathrm{Hg}_{1}$ has exactly 2 .

The dihedral group is only half the story; the other half is the affine algebra data $B_{m, 2}$. Its modular invariants were classified in [32]: when $\mu$ is not a perfect square (the situation here), the complete list is $B\left(d_{0}, \ell_{0}\right)$ and $B\left(d_{1}, \ell_{1} ; d_{2}, \ell_{2}\right)=B\left(d_{2}, \ell_{2} ; d_{1}, \ell_{1}\right)$ where $d_{i}|\mu, \mu| d_{i}^{2}$, and $1 \leq \ell_{i} \leq m$ obeys $\ell_{i}^{2} \equiv_{d_{i}^{2} / \mu} 1$. for matrices $B(d, \ell)$ and $B\left(d_{1}, \ell_{1} ; d_{2}, \ell_{2}\right)$ defined in 32]. Those modular invariants which are permutation matrices are the $B(\mu, \ell)$ for $1 \leq \ell \leq m$ satisfying $\ell^{2} \equiv_{\mu} 1$ : their nonzero entries are $B(\mu, \ell)_{z, z}=1$ for $z \in\left\{0, \mathfrak{b}, \mathfrak{a}_{i}\right\}$, and $B(\mu, \ell)_{\mathfrak{d}_{l}, \mathfrak{d}_{l^{\prime}}}=1$ for $l^{\prime} \equiv_{\mu} \pm \ell l$. We see from this and Theorem 3 that the modular invariants of $\mathcal{D}^{\omega} \mathrm{Hg}_{\nu}$ come from those of $\mathcal{D}^{-, \omega} \mathrm{Hg}_{\nu}$ and $B_{m, 2}$ in a very direct sense.

Recall [53, 22] that the sufferable modular invariants of the double of a finite group $G$ are parametrised by pairs $(H, \psi)$ where $H$ is a subgroup of $G \times G$ and $\psi \in H^{2}\left(B_{H} ; \mathbb{C}^{\times}\right)$is called discrete torsion. This suggests:

Question 4. Find some analogue for $\mathcal{D}^{0} \mathrm{Hg}_{\nu}$ of this $(H, \psi)$ parametrisation of sufferable modular invariants.

This $(H, \psi)$ parametrisation belongs most naturally to the $\mathcal{D} \Delta$ formulation of the double of the group $G$. As mentioned in Section 1.2, $\mathcal{D} \widehat{\Delta}$ is also the double of $G$. Is there also a parametrisation of the sufferable modular invariants of the double of $G$ which is more natural in the $\mathcal{D} \widehat{\Delta}$ language?

Of special importance (recall Section 1.3) are the monomial modular invariants of $\mathcal{D}^{0} \mathrm{Hg}_{\nu}$. Three of these are obvious:

$$
\left|c h_{0}+c h_{\mathfrak{b}}+2 \sum c h_{\mathfrak{a}_{i}}\right|^{2},\left|c h_{0}+c h_{\mathfrak{b}}+\sum c h_{\mathfrak{a}_{i}}+\sum c h_{\mathfrak{c}_{h, 0}}\right|^{2},\left|c h_{0}+c h_{\mathfrak{b}}+2 \sum c h_{\mathfrak{c}_{h, 0}}\right|^{2}
$$

Proposition 4. When $\nu=p$ or pq for (not necessarily distinct) primes $p, q$, the only monomial modular invariants are the three in (3.6).

Proof. We need to find all eigenvectors $u$ of $S, T$ with eigenvalue 1 , with $u_{0}=1$ and all other $u_{x} \in\{0,1,2, \ldots\}$, so $Z=\left|\sum_{x} u_{x} c h_{x}\right|^{2}$. We know $u_{\mathfrak{b}}=1$ since $M_{\mathfrak{b}, \mathfrak{b}}=1$ for any modular invariant (clear from the proof of Theorem 3), hence $u_{\mathfrak{d}_{l}}=0$ for all $l$. We need to determine $u_{i}:=u_{\mathfrak{a}_{i}}$ and $u_{h, j}:=u_{\mathfrak{c}_{h, j}}$.

$T u=u$ implies $u_{h, j}=0$ unless $\nu$ divides $h j$. Therefore if $u_{h, j} \neq 0$ we have:

(i) for $\nu$ prime, $j$ must be 0 ;

(ii) for $\nu=p^{2}$, only for $j=0$ or $p \mid h$ and $p \mid j$;

(iii) for $\nu=p q(p \neq q)$, only for $j=0$, or $p \mid h$ and $q \mid j$, or $q \mid h$ and $p \mid j$. 
$S u=u$ implies, for all $1 \leq h, h^{\prime \prime} \leq n$ and $0 \leq i<\nu$,

$$
\begin{array}{r}
2+2 \sum u_{j}+2 \sum u_{h^{\prime}, j} \cos \left(2 \pi i h^{\prime} / \nu\right)=\nu u_{i}, \\
2+2 \sum \cos (2 \pi j h / \nu)+2 \sum \cos \left(2 \pi\left(h j+h^{\prime} i\right) / \nu\right) u_{h^{\prime}, j}=\nu u_{h, i}, \\
\sum \cos (2 \pi h j / \nu) u_{h^{\prime \prime}, j}=\sum \cos \left(2 \pi h^{\prime \prime} j / \nu\right) u_{h, j},
\end{array}
$$

where we sum over $h^{\prime}, j$ ((3.9) was obtained by hitting (3.8) with $\cos \left(2 \pi h^{\prime \prime} i / \nu\right)$ and summing over $i$ ). In analysing these equations, it is useful to recall (for $k$ odd)

$$
\sum \xi_{k}^{\ell}=2 \sum \cos (2 \pi l / k)=\mu(k)
$$

the sums taken over the $1 \leq \ell \leq k$ and $1 \leq l<k / 2$ which are coprime to $k$. The Möbius function $\mu(k)$ equals $(-1)^{n}$ if $k$ is a product of $n \geq 0$ distinct primes, and 0 otherwise. The Galois symmetry $\sigma_{\ell}$ for $\ell \in \mathbb{Z}_{\nu}^{\times}$(recall (1.11)) maps $\mathfrak{a}_{i}$ to $\mathfrak{a}_{ \pm \ell i}$ and $\mathfrak{c}_{h, j}$ to $\mathfrak{c}_{ \pm \ell h, \ell j}$. Therefore $u_{i}=u_{\operatorname{gcd}(i, \nu)}$ and $u_{h, j}=u_{\operatorname{gcd}(h, \nu), j h / \operatorname{gcd}(h, \nu)}$, so $u_{h,-j}=u_{h, j}$.

For $\nu$ prime, this means $u_{i}=u_{1}$ and $u_{h, j}=u_{1,0} \delta_{j, 0}$. Plugging this into (3.7) gives $u_{1}+u_{1,0}=2$, which correspond to the three solutions (3.6).

For $\nu=p^{2}$, we read off from (3.9) with $h=p, h^{\prime}=1$ that $u_{p, j p}=u_{p, 0}-u_{1,0}$ for all $j \neq 0$. Then comparing (3.7) for $i=1, p$ gives $2=u_{p}+u_{1,0}=u_{1}+u_{p, 0}$. We can now force $u_{p}=u_{1}$ by (3.8) at $h=p, i=1$, and again we recover (3.6).

Finally, turn to $\nu=p q$. (3.9) with $h=1$ and $h^{\prime}=p, q$ gives respectively $u_{p, q j}=$ $u_{p, 0}-u_{1,0}, u_{q, p j}=u_{q, 0}-u_{1,0}$ for $j \neq 0$. Then (3.7) at $i=1, p, q$ gives $u_{q}+u_{q, 0}=$ $u_{p}+u_{p, 0}=2$ and $u_{1,0}=2+u_{1}-u_{p}-u_{q}$. Now (3.8) at $h=i=p$ and $h=i=q$ give $u_{1}=u_{p}=u_{q}$ and $u_{1,0}=u_{p, 0}=u_{q, 0}$ and we're done. QED

The $\nu=3$ modular invariants (2.3), (2.4) generalise, for any $\mathcal{D}^{ \pm, \omega} D_{\nu}, \mathcal{D}^{\omega} \mathrm{Hg}_{\nu}$, to

$$
\left|c h_{0}+c h_{\mathfrak{b}}\right|^{2}+2 \sum\left|c h_{\mathfrak{a}_{i}}\right|^{2}+2 \sum\left|c h_{\mathfrak{c}_{h, j}}\right|^{2} .
$$

This corresponds to the $\mathbb{Z}_{\nu}$ subsystem in both $\mathcal{D} D_{\nu}$ and $\mathcal{D}^{0} \mathrm{Hg}_{\nu}$, and to the VOA realising $\mathcal{D}^{\omega} \mathbb{Z}_{\nu}$ and containing both $\mathcal{D}^{+, \omega} D_{\nu}$ and $\mathcal{D}^{\omega} \mathrm{Hg}_{\nu}$ VOAs.

\section{Subfactors for Haagerup-Izumi modular data}

\subsection{Izumi's subfactors and their modular data}

In Section 5 we address the question of realising this generalised Haagerup modular data $\mathcal{D}^{\omega} \mathrm{Hg}_{\nu}$ by VOAs. In this section we address their subfactor realisation.

In Section 7 of [38, Izumi suggests associating subfactors to any odd abelian group $K$ of order $\nu$, using endomorphisms in the Cuntz algebra $\mathcal{O}_{\nu+1}$. (Warning: Izumi's $n$ is our $\nu=2 n+1$.) Let $\mathcal{A}(K)$ be the set of all $\nu \times \nu$ complex matrices 
$A=\left(A_{g, h}\right), g, h \in K$, satisfying

$$
\begin{aligned}
& A_{g, h}=\overline{A_{h, g}}, A_{g, h}=A_{-h, g-h}=A_{h-g,-g}, A_{g, 0}=\delta_{g, 0}-1 /(\delta-1), \\
& \sum_{m \in K} A_{g+m, h} \overline{A_{m, h}}=\delta_{g, 0}-\delta_{h, 0} / \delta, \\
& \sum_{m \in K} A_{m, g+h} \overline{A_{m+k, g} A_{m+l, h}}=A_{g+l, k} A_{h+k, l}-\delta_{g, 0} \delta_{h, 0} / \delta,
\end{aligned}
$$

for all $g, h, k, l \in K\left(\right.$ recall $\left.\delta=\left(\nu+\sqrt{\nu^{2}+4}\right) / 2\right)$. These equations imply [38]

$$
\begin{aligned}
& \left|A_{g, h}\right|=\sqrt{\delta}, \\
& A_{g+k, h} A_{h, k}=A_{-k, g} A_{g, h-k},
\end{aligned}
$$

for any $g, h, k \in K$ with $g \neq 0, h \neq 0, g \neq h$. Izumi shows that to any matrix $A \in \mathcal{A}(K)$, there corresponds a (nonbraided) subfactor of index $\delta+1$ with principal graph the $\nu$-star (see the top graphs of Figures 2 and 4 ). We call these subfactors of Izumi type $K$. Next subsection we determine much of the data of these subfactors. His Theorem 8.4 identifies the modular data $S, T$ of the even part of the quantum double of this subfactor in terms of $m\left(\nu^{2}+1\right)$ variables $\omega_{l}, C_{g, h}^{l}, g, h \in K, 1 \leq l \leq m$, satisfying the $m\left(\nu^{2}+1\right)$ equations:

$$
\sum_{g \in K} C_{0, g}^{l}=\omega_{l}-\overline{\omega_{l}} / \delta, \omega_{l} C_{g, h}^{l}-\sum_{k \in K} A_{g+k, 2 h} C_{h, k}^{l}=\delta_{h, 0} \overline{\omega_{l}} / \delta
$$

Some solutions to (4.6), occurring when $\omega_{l}^{\nu}=1$, are redundant (i.e. correspond to $\mathfrak{b}, \mathfrak{a}_{i}$ or $\mathfrak{c}_{h, j}$ ) and should be ignored. In particular, precisely $\sigma_{1}(\nu)-1$ (i.e. the sum of divisors $d>1$ of $\nu$ ) solutions to (4.6) with $\omega_{l}=1$ are redundant, and for any other root of unity $\omega_{l}$ of order $d$ dividing $\nu$, the number of redundant solutions is $\sum d^{\prime} \phi\left(\nu / d^{\prime}\right) / 2$ where $\phi$ is Euler's totient and the sum is over all divisors $d^{\prime}<d$ of $d$. The entries of the $S$ and $T$ matrices equal those of $\mathcal{D}^{0} \mathrm{Hg}_{\nu}$ except possibly for those in the bottom-right $m \times m$ block, which are given by $T_{\mathfrak{d}_{l}, \mathfrak{d}_{l}}=e^{-2 \pi 1 c / 24} \omega_{l}$ and

$$
S_{\mathfrak{v}_{l}, \mathfrak{d}_{l^{\prime}}}=\frac{1}{\nu \delta+2}\left(\omega_{l} \omega_{l^{\prime}}+\delta \sum_{g, h \in K} \overline{C_{g, g+h}^{l} C_{-g, h}^{l^{\prime}}}\right) .
$$

When some $\omega_{l}$ has more than one solution in (4.6), (4.7) is ambiguous as it isn't obvious which solutions to (4.6) to use.

Izumi shows in his Example 7.1 that $\mathcal{A}\left(\mathbb{Z}_{3}\right)$ contains exactly two matrices, both corresponding to the Haagerup subfactor. In Appendix $\mathrm{C}$ he exactly solves (4.6) for $A \in \mathcal{A}\left(\mathbb{Z}_{3}\right)$, and in this way obtains (a complicated expression for) $\mathcal{D} H g$.

In his Example 7.2, Izumi shows $\mathcal{A}\left(\mathbb{Z}_{5}\right)$ contains exactly 4 matrices, again corresponding to a single (new) subfactor. Izumi does not solve (4.6) for them. This analysis of (4.1)-(4.3) for $\nu=3$ and $\nu=5$ is as far as Izumi went (this is what we mean when we call it a hypothetical family of subfactors). 
Nevertheless we can push a little further this analysis. The group $\operatorname{Aut}(K)$ acts naturally on the matrix $A$ by shuffling the entries: $\alpha \in \operatorname{Aut}(K)$ sends $A$ to $A^{\alpha}$ defined by $A_{g, h}^{\alpha}=A_{\alpha g, \alpha h}$. For example, when $K=\mathbb{Z}_{\nu}$, Aut $(K)=\mathbb{Z}_{\nu}^{\times}$acts by multiplication. In this way, given any $A \in \mathcal{A}(K)$, Aut $(K)$ embeds into $\operatorname{Aut}(\mathbb{Q}[A])$ and fixes $\delta$, where $\mathbb{Q}[A]$ is the field generated over $\mathbb{Q}$ by all entries $A_{g, h}$. Call $A, A^{\prime} \in \mathcal{A}(K)$ equivalent if $A^{\prime}=A^{\alpha}$ for some $\alpha \in \operatorname{Aut}(K)$. Equivalent matrices give rise to equivalent subfactors and equivalent modular data. For later convenience, for $x \in \mathbb{Q}[A]$, write $\operatorname{Tr} x$ for the orbit sum $\sum_{y \in\langle x\rangle} y$ over the $K$-orbit $\langle x\rangle$.

It is very difficult to solve (4.6) directly (see Appendix $\mathrm{C}$ of [38] for the $\nu=3$ argument, which is already quite involved). Our strategy for finding the modular data is to reduce it to computer calculations. A key observation is that if $S, T$ are the modular data of $\mathcal{D}^{0} \mathrm{Hg}_{\nu}$, and if $S^{\prime}, T$ is a second modular data, with

$$
\left|S_{\mathfrak{o}_{l}, \mathfrak{o}_{l^{\prime}}}^{\prime}-S_{\mathfrak{d}_{l}, \mathfrak{o}_{l^{\prime}}}\right|<\frac{1}{18 \sqrt{\mu}}
$$

for all $1 \leq l, l^{\prime} \leq m$, and all other entries of $S^{\prime}$ and $S$ are equal, then (1.3) implies $S^{\prime}=S$ everywhere. Maple 11 (x86 64 Linux) was used in these calculations. It is difficult to rigourously analyse the resulting error, but various consistency checks (e.g. unitarity of the numerically obtained $S^{\prime}$ matrix, or the values of $\left|S_{\mathfrak{d}_{l}, \mathfrak{o}_{l^{\prime}}}^{\prime}-S_{\mathfrak{d}_{l}, \mathfrak{d}_{l^{\prime}}}\right|$ ) all indicate it is on the order of $10^{-7}$ or better, far more precision than is actually needed. More details are given below. Computer calculations were not used in the determination of $\mathcal{A}(K)$ in Theorem 5 .

Theorem 5. (a) There is a unique $A \in \mathcal{A}(1)$. Its modular data is $\mathcal{D}^{0} \mathrm{Hg}_{1}$.

(b) There are precisely 2 matrices in $\mathcal{A}\left(\mathbb{Z}_{3}\right)$. They are equivalent and realise $\mathcal{D}^{0} \mathrm{Hg}_{3}$.

(c) There are precisely 4 matrices in $\mathcal{A}\left(\mathbb{Z}_{5}\right)$. They are equivalent and realise $\mathcal{D}^{0} \mathrm{Hg}_{5}$.

(d) There are precisely 6 matrices in $\mathcal{A}\left(\mathbb{Z}_{7}\right)$. They are equivalent and realise $\mathcal{D}^{0} \mathrm{Hg}_{7}$.

(e) There are precisely 12 matrices in $\mathcal{A}\left(\mathbb{Z}_{9}\right)$. They form 2 equivalence classes and define inequivalent subfactors; one realises the $T$ matrix of $\mathcal{D}^{0} \mathrm{Hg}_{9}$, and the other realises the $T$ of $\mathcal{D}^{0,\{5,17\}} \mathrm{Hg}_{9}$ (defined in Section 3.3).

(f) $\mathcal{A}\left(\mathbb{Z}_{3} \times \mathbb{Z}_{3}\right)$ is empty.

Proof. Let's begin by identifying $\mathcal{A}(K)$ for these groups (done in [38] for $K=\mathbb{Z}_{3}, \mathbb{Z}_{5}$ ). It is trivial that $\mathcal{A}(1)=\left\{\left(\frac{\delta-2}{\delta-1}\right)\right\}$, and for $\mathbb{Z}_{3}$ and $\mathbb{Z}_{5}$ (4.1) fixes everything in terms of complex numbers $a$ and $a, b$, respectively:

$$
A\left(\mathbb{Z}_{3}\right)=\frac{1}{\delta-1}\left(\begin{array}{ccc}
\delta-2 & -1 & -1 \\
-1 & -1 & a \\
-1 & \bar{a} & -1
\end{array}\right), A\left(\mathbb{Z}_{5}\right)=\frac{1}{\delta-1}\left(\begin{array}{ccccc}
\delta-2 & -1 & -1 & -1 & -1 \\
-1 & -1 & a & b & a \\
-1 & \bar{a} & -1 & b & b \\
-1 & \bar{b} & \bar{b} & -1 & a \\
-1 & \bar{a} & \bar{b} & \bar{a} & -1
\end{array}\right)
$$

For $\nu=3$, (4.3) forces $a^{2}-a+\delta=0$. The two roots of this polynomial are complex conjugates, and indeed $-1 \in \mathbb{Z}_{3}^{\times}$here acts as $a \leftrightarrow \bar{a}$. Similarly, the generator $2 \in \mathbb{Z}_{5}^{\times}$ acts on $A\left(\mathbb{Z}_{5}\right)$ by sending $a \mapsto b \mapsto \bar{a} \mapsto \bar{b} \mapsto a$. Then $a$ has minimal polynomial 
$x^{4}-\delta x^{3}+(3 \delta-2) x^{2}-\delta^{2} x+\delta^{2}=0$ and the other 3 roots of this polynomial are $\bar{a}, b, \bar{b}$ - indeed, the Galois group over $\mathbb{Q}[\delta]$ of this polynomial is $\mathbb{Z}_{5}^{\times}$with this action. Given $a$, this and the equation $1-a-\bar{a}+a \bar{b}+\bar{a} b=0$ then determine $b$.

Computation of $\mathcal{A}\left(\mathbb{Z}_{7}\right)$ is handled similarly. (4.1) and (4.5) give us

$$
A\left(\mathbb{Z}_{7}\right)=\frac{1}{\delta-1}\left(\begin{array}{ccccccc}
\delta-2 & -1 & -1 & -1 & -1 & -1 & -1 \\
-1 & -1 & a & b & c & b & a \\
-1 & \bar{a} & -1 & b & d & d & b \\
-1 & \bar{b} & \bar{b} & -1 & c & d & c \\
-1 & \bar{c} & \bar{d} & \bar{c} & -1 & b & b \\
-1 & \bar{b} & \bar{d} & \bar{d} & \bar{b} & -1 & a \\
-1 & \bar{a} & \bar{b} & \bar{c} & \bar{b} & \bar{a} & -1
\end{array}\right)
$$

The generator $3 \in \mathbb{Z}_{7}^{\times}$sends $a \mapsto c \mapsto d \mapsto \bar{a} \mapsto \bar{c} \mapsto \bar{d} \mapsto a$ and $b \mapsto \bar{b} \mapsto b$. (4.5) gives $a d=b c$, while (4.2) with $(g, h)=(1,1),(3,1),(2,3)$ give

$$
(b+\bar{b}+2)(c+\bar{c})=-1+\operatorname{Tr} a=(b+\bar{b}+2)(a+\bar{a})=(b+\bar{b}+2)(d+\bar{d}),
$$

where the last two equalities come from the $\mathbb{Z}_{7}^{\times}$symmetry. If $b+\bar{b} \neq-2$, then $a=d=\bar{c}$ and $\delta b=a^{3}$ and we get two incompatible equations for $s:=a+\bar{a}$, namely $s^{4}+s^{2}(\delta-4)-\delta s=\delta-2$ and $s^{3}+(\delta-3) s-\delta s^{2}=5 \delta+1$.

Thus $b+\bar{b}=-2$ (hence $b=-1 \pm 1 \sqrt{\delta-1}$ ) and $\operatorname{Tr} a=1$. Let $t=a+d+\bar{c}$. Then $2 \operatorname{Re}(t)=1$ and (from (4.2) $) 2 \operatorname{Re}(t \bar{b})=-3 \delta-5$ gives $2 t=1+(b+1)(3-\delta)$ and we obtain $a^{3}-t a^{2}+b \bar{t} a-\delta b=0$. The other two roots of this polynomial are $d$ and $\bar{c}$ (which to call $d$ follows from $2 \operatorname{Re}(b+c-a \bar{b})=\delta$ ), and its Galois group over $\mathbb{Q}[\delta, b]$ is $\left\langle 3^{2}\right\rangle<\mathbb{Z}_{7}^{\times}$.

Computation of $\mathcal{A}\left(\mathbb{Z}_{9}\right)$. We get as before

$$
A\left(\mathbb{Z}_{9}\right)=\frac{1}{\delta-1}\left(\begin{array}{ccccccccc}
\delta-2 & -1 & -1 & -1 & -1 & -1 & -1 & -1 & -1 \\
-1 & -1 & a & b & c & d & c & b & a \\
-1 & \bar{a} & -1 & b & e & f & f & e & b \\
-1 & \bar{b} & \bar{b} & -1 & c & f & g & f & c \\
-1 & \bar{c} & \bar{e} & \bar{c} & -1 & d & f & f & d \\
-1 & \bar{d} & \bar{f} & \bar{f} & \bar{d} & -1 & c & e & c \\
-1 & \bar{c} & \bar{f} & \bar{g} & \bar{f} & \bar{c} & -1 & b & b \\
-1 & \bar{b} & \bar{e} & \bar{f} & \bar{f} & \bar{e} & \bar{b} & -1 & a \\
-1 & \bar{a} & \bar{b} & \bar{c} & \bar{d} & \bar{c} & \bar{b} & \bar{a} & -1
\end{array}\right) .
$$

Moreover, (4.5) yields $g b=c f$ and $a \bar{c}=b \bar{e}=d \bar{f}$. The generator $2 \in \mathbb{Z}_{9}^{\times}$sends $a \mapsto e \mapsto d \mapsto \bar{a} \mapsto \bar{e} \mapsto \bar{d} \mapsto a, b \mapsto f \mapsto \bar{c} \mapsto \bar{b} \mapsto \bar{f} \mapsto c \mapsto b$, and $g \mapsto \bar{g} \mapsto g$.

Up to the relations $g b=c f, a \bar{c}=b \bar{e}=d \bar{f}$, there are precisely $17 \mathbb{Z}_{9}^{\times}$orbits in $b, \bar{b}, c, \bar{c}, f, \bar{f}$ of degree $\leq 3$. Using (4.2) with $g=3$ and (4.3) when 3 divides both $g$ and $h$, we can compute these 17 orbit sums $\operatorname{Tr}$ as linear expressions in $s:=\operatorname{Tr} b=$ $2 \operatorname{Re}(b+c+f)$. For example, $\operatorname{Tr} g=1-s, \operatorname{Tr}(b g)=3(s-\delta), \operatorname{Tr}(b \bar{g})=2 \delta+1+s(2-\delta)$. But $\operatorname{Tr} b \operatorname{Tr} g=\operatorname{Tr}(b g)+\operatorname{Tr}(b \bar{g})$, so $s^{2}+(4-\delta) s+1-\delta=0$. Choose one of these 2 solutions for $s$ (they both yield $A \in \mathcal{A}\left(\mathbb{Z}_{9}\right)$ ).

From $g+\bar{g}=1-s$ we get $g=(1-s \pm \sqrt{(\delta-6) s-3 \delta}) / 2$. Again select one of these $g$ (they are complex conjugates). Let $t:=b+\bar{c}+\bar{f}$. Then $t+\bar{t}=s$ and $t g+\overline{t g}=3(s-\delta)$, which fixes $t=s / 2 \pm(3 \delta-6-\delta s-s) \sqrt{(\delta-6) s-3 \delta} / 36$ (use the same sign as in $g$ ). Then $b \bar{c}+b \bar{f}+\bar{c} \bar{f}=\overline{t g}$ and $b \overline{c f}=\delta \bar{g}$, so $b, \bar{c}, \bar{f}$ are the 3 roots of 
$x^{3}-t x^{2}+\overline{g t} x-\delta \bar{g}=0$. Let $b$ be any of these roots; which to call $\bar{c}$ and $\bar{f}$ is fixed by the relation $\operatorname{Tr}\left(b^{2} \bar{c}\right)=6 \delta+3+(8-\delta) s$.

Now (4.2) at $(g, h)=(1,1)$ and $(4,2)$ gives $a^{2}+v a / u+\delta \bar{u} / u=0$ for $(u, v)=$ $\left(\bar{b}+\bar{c}^{2} / \bar{f}-1,1+\bar{b} c+b \bar{c}\right)$ and $(f+\bar{c}-\overline{b c} / \delta, \delta-f-\bar{f})$ respectively; subtracting these expresses $a$ rationally in terms of $b, c, f$. Then $d=a f / c$ and $e=b c / a$. These values of $a, \ldots, g$ will solve (4.1)-(4.3). There are 2 choices for $s, 2$ for $g$, and 3 for $b$. The $\mathbb{Z}_{9}^{\times}$symmetry accounts for this $g$ and $b$ ambiguity, leaving 2 inequivalent solutions.

Computation of $\mathcal{A}\left(\mathbb{Z}_{3}^{2}\right)$. Write the (1,0)-row of the matrix $(\delta-1) A\left(\mathbb{Z}_{3}^{2}\right)$ as $(-1,-1, t, u, v, w, x, y, z)$. (4.5) with $g=h=(1,0)$ and $k=(0,1),(1,1)$ gives $y \bar{u}=x \bar{v}=z \bar{w}=: \delta a$ for some complex number $a$ with $|a|=1$. From (4.5) with $g=(1,1), h=(1,0), k=(1,2)$ yields $\delta t=x y z$, and applying the $\mathbb{Z}_{3}^{2}$-automorphism $(i, j) \mapsto(i,-j)$ to this we get $\delta t=u v w$. Comparing these gives $a^{3}=1$.

Putting $g=(0,1), h=(1,0)$ into (4.2) yields

$$
1-\delta=(\bar{a} u+a \bar{u}-1)(\bar{a} v+a \bar{v}-1) .
$$

Hitting this with the $\mathbb{Z}_{3}^{2}$-automorphism $(i, j) \mapsto(i+j, j)$ gives the same equation with $w$ replacing $u$. Thus $\bar{a} u+a \bar{u}-1=\bar{a} v+a \bar{v}-1$ and (4.11) says there is a real number whose square is negative. This impossibility establishes part (f).

Determining the modular data. Consider first $K=\mathbb{Z}_{3}$ and fix $A \in \mathcal{A}\left(\mathbb{Z}_{3}\right)$. Let $\omega_{l}, C_{g, h}^{l}$ be some solution to (4.6). Then we learn from the above proof that any automorphism $\sigma \in \operatorname{Gal}(\overline{\mathbb{Q}} / \mathbb{Q}[\delta])$ corresponds to an $\ell \in \mathbb{Z}_{3}^{\times}$in such a way that $\sigma A_{g, h}=$ $A_{\ell g, \ell h}$. Certainly $\sigma$ sends $\omega_{l}$ to some other root of unity $w_{l^{\sigma}}$ of the same order $d$, and moreover $\sigma \overline{\omega_{l}}=\overline{\omega_{l^{\sigma}}}$ (since $\sigma$ commutes with complex conjugate in cyclotomic fields). Hence $\sigma$ sends the solution $\omega_{l}, C_{g, h}^{l}$ of (4.6) to another solution $\omega_{l^{\sigma}}, C_{g, h}^{l^{\sigma}}$ given by $\sigma\left(C_{g, h}^{l}\right)=C_{\ell g, \ell h}^{l^{\sigma}}$. Incidentally, (4.7) then implies the Galois actions

$$
\sigma\left(S_{\mathfrak{d}_{l}, \mathfrak{d}_{l^{\prime}}}\right)=S_{\mathfrak{d}_{l} \sigma, \mathfrak{d}_{l^{\prime}}}, \sigma\left(T_{\mathfrak{d}_{l}, \mathfrak{o}_{l}}\right)=T_{\mathfrak{d}_{l} \sigma, \mathfrak{d}_{l} \sigma}
$$

(the second equation is simply the statement $\sigma \omega_{l}=\omega_{l^{\sigma}}$; to see the first, compute instead $\sigma \bar{S}$ ). This is stronger than the usual Galois action (1.4) in modular data, though of course it is compatible with $\mathcal{D}^{0} \mathrm{Hg}_{\nu}$.

Since $\delta \in \mathbb{Q}\left[\xi_{\mu}\right]$, when $\mu$ is coprime to the order $d$ of $\omega_{l}$ any automorphism in $\operatorname{Gal}\left(\mathbb{Q}\left[\xi_{d}\right] / \mathbb{Q}\right)$ will lift to a $\sigma$ fixing $\delta$ (when $\operatorname{gcd}(d, \mu)>1$, only half will). So if (4.6) can be solved for a $d$ th root of unity $w_{l}$, for $d$ coprime to $\mu$, then it can be solved for all $d$ th roots of 1 . That is, we would have at least $\phi(d)$ solutions. As long as $d$ doesn't divide $\nu$, none of these are redundant. But Izumi tells us there are precisely $m$ nonredundant (independent) solutions to (4.6) (corresponding to the $m$ half-braidings, giving rise to the $m$ primaries of type $\mathfrak{d}_{l}$ ).

Hence any solution to (4.6) involves $\omega_{l}$ of order $d$ where $\phi(d) \leq m$ (for $d$ coprime to $\mu$ ) or $\phi(d) \leq 2 m$ (otherwise). There is thus a finite number of possibilities for $\omega_{l}$. A little thought reduces the number further - e.g. when $\operatorname{gcd}(d, \mu)=1$ it suffices to consider only $\omega_{l}=\xi_{d}$. We find that (for $\nu=3$ ) only 12 possibilities for $\omega_{l}$ need be considered, namely $\pm 1, \pm \xi_{3}, 1, \pm \xi_{7}, \pm \xi_{9}, \pm \xi_{13},-\xi_{13}^{6}$. To rule out these possibilities, 
we compute determinants. In particular, for $\omega_{l} \neq 1, \xi_{3}$, we show that the linear system (4.6) is inconsistent by showing the $\left(\nu^{2}+1\right) \times\left(\nu^{2}+1\right)$ augmented matrix (formed from the coefficients and constant vector) has nonzero determinant. To show $\omega_{l}=1$ (respectively $\omega_{l}=\xi_{3}$ ) has at most 3 (resp. 1) independent solutions, we deleted 3 (resp. 1) equations and 2 (resp. 0) variables from (4.6) so that the resulting reduced coefficient matrix has nonzero determinant. We evaluated these determinants numerically using Maple. If some determinant was fairly close to 0, we found some Galois associate of $\omega_{l}$ with large determinant (i.e. of order 1 or higher).

In this way we show that $\omega_{l}$ must be one of the values $\xi_{\mu}^{m l^{2}}$. We can now determine the modular data, rigourously identifying it with $\mathcal{D}^{0} \mathrm{Hg}_{\nu}$, as follows. Numerically compute the solution to (4.6) and plug it into (4.7) to obtain an estimate $S^{\prime}$. We find that $S^{\prime}$ matches $S$ to within $2 \times 10^{-9}$, well within the 0.015 required by (4.8).

This argument for $K=\mathbb{Z}_{5}$ and $\mathbb{Z}_{7}$ is identical. For them exactly 14 and 22 , respectively, values of $\omega_{l}$ need to be considered. The error $\left|S^{\prime}-S\right|$ is less than $2 \times 10^{-9}$ and $5 \times 10^{-8}$ respectively, again well within (4.8).

The modular data calculation for $K=\mathbb{Z}_{9}$ is more complicated for two reasons, both related to the compositeness of $\mu=5 \cdot 17$. Fix $A \in \mathcal{A}\left(\mathbb{Z}_{9}\right)$ (the result for the other $A$ is automatic by Galois considerations). First, an automorphism $\sigma$ will permute the entries of $A$ iff $\sigma$ fixes both $\delta$ and $s=\operatorname{Tr} b$ (incidentally, $\operatorname{Gal}(\mathbb{Q}[s] / \mathbb{Q}) \cong \mathbb{Z}_{2}^{2}$, so $s$ lies in a cyclotomic extension of $\mathbb{Q})$. Hence potentially only $1 / 4$ of $\operatorname{Gal}\left(\mathbb{Q}\left[\xi_{d}\right] / \mathbb{Q}\right)$ lifts to such $\sigma$. This means more possibilities for $\omega_{l}$ to be eliminated (218 possibilities is overkill), and we find that the $T$ matrix for $A$ exactly matches that of either $\mathcal{D}^{0} \mathrm{Hg}_{9}$ or $\mathcal{D}^{0,\{5,17\}} \mathrm{Hg}_{9}$. More significantly, because of equalities like $\omega_{1}=\omega_{16}$, there won't always be unique solutions to (4.6), and (4.7) is ambiguous. This means we cannot identify $S$ without more work. QED

The key difference between $K=\mathbb{Z}_{9}$ and $\mathbb{Z}_{3}^{2}$ is the size of their automorphism groups ( 6 vrs 48 ). Nonuniqueness for $K=\mathbb{Z}_{9}$ arises because $\mu=85$ is composite: more generally, where $k>1$ is the number of distinct prime divisors of $\mu$, there will be at least $\left(\begin{array}{l}k \\ 2\end{array}\right)$ inequivalent $A$ and indeed inequivalent subfactors, realising the inequivalent modular data of type $\mathcal{D}^{0, P} \mathrm{Hg}_{\nu}$ defined in Section 3.3.

This nonuniqueness of subfactors with identical principal graphs is not a surprise: for a simple example, the principal graph fails to uniquely identify the subfactor even in index $<4$ (two subfactors realise each of the graphs $E_{6}, E_{8}$ although one is the opposite of the other) whilst in index 4 the affine graphs $A_{2 n-5}^{(1)}$ and its orbifold $D_{n}^{(1)}$ both have $n-2$ inequivalent subfactors [39].

Exact expressions for the matrices $A \in \mathcal{A}(K)$ appear in the proof of Theorem 5 . Numerical estimates for these $A$ may also be of interest. A convenient way to express this, for $K$ cyclic, is in terms of $j_{2}, j_{3}, \ldots, j_{n+1} \in \mathbb{R}$ : for $0<g<h<\nu$ we have

$$
A_{g, h}=\frac{\sqrt{\delta}}{\delta-1} \exp \left[1\left(j_{h}-j_{g}-j_{h-g}\right)\right]
$$


where $j_{1}=0$ and $j_{n+1+i}=j_{n+1}+j_{n}-j_{n-i}$ for $1 \leq i<n$ (see Lemma 7.3 of [38]).

$$
\begin{aligned}
j_{2}^{(3)} & \approx 1.292076 \\
\left(j_{2}^{(5)}, j_{3}^{(5)}\right) & \approx(0.1846862,1.5984702) ; \\
\left(j_{2}^{(7)}, j_{3}^{(7)}, j_{4}^{(7)}\right) & \approx(2.471228,0.51685555,0.2137724) ; \\
\left(j_{2}^{(9)}, \ldots, j_{5}^{(9)}\right) & \approx(2.396976693,2.079251103,-0.2079168419,-2.508673987) \\
\left(j_{2}^{(9) \prime}, \ldots, j_{5}^{(9) \prime}\right) & \approx(-2.364737070,1.031057162,1.569692175,0.3383837765) .
\end{aligned}
$$

$j^{(9)}$ corresponds to $\mathcal{D}^{0} \mathrm{Hg}_{9}$ and $j^{(9) \prime}$ to $\mathcal{D}^{0,\{5,17\}} \mathrm{Hg}_{9}$.

It should be possible to identify $\mathcal{A}\left(\mathbb{Z}_{\nu}\right)$ for the next couple $\nu$ 's, using the method of $\nu=9$ described in the proof. To probe the answer, we used Maple to obtain numerical solutions to (4.1)-(4.3). We don't have any proof that these correspond to actual solutions (although the numerics are convincing), and we certainly have no proof that there are no other solutions for these $\nu$. The inequivalent numerical solutions we obtained for $K=\mathbb{Z}_{\nu}, 11 \leq \nu \leq 19$, are:

$$
\begin{aligned}
& j^{(11)} \approx(0.9996507,2.7258434,-0.5714203,-1.7797340,1.2675985) \\
& j^{(11) \prime} \approx(-2.6444397,-1.7629598,-2.6444440,2.7572657,0.1128260) ; \\
& j^{(13)} \approx(-3.1050384,0.5993399,-0.111708,-0.969766,1.336848,1.00483129) ; \\
& j^{(15)} \approx(-1.0777623,-.7748018,-2.171863,-1.6068402,-.257508,2.092502, .72289565) ; \\
& j^{(17)} \approx(-1.466074, .291489,3.130735,-2.693185,1.398153,-.611938,-1.667078,-1.754821) ; \\
& j^{(19)} \approx(-2.677465,1.088972,-.899442, .015448,-1.240928,-.493394,1.839879 \\
&\quad-1.525884,-2.084374) ; \\
& j^{(19) \prime} \approx(.896858,-.882585,-2.369855,-1.873294,-1.711620,-.119360,2.972018 \\
&\quad-2.460652, .041334) .
\end{aligned}
$$

For $\nu=13,15,17$ respectively, $\mu=173,229,293$ is prime. In these cases, choosing $\omega_{l}=\xi_{\mu}^{m l^{2}}$ yields a unique solution to (4.6) (according to Maple) and plugging into (4.7) yields a very close approximation (of order $10^{-6}$ or so) $S^{\prime}$ to the $S$ matrix of $\mathcal{D}^{0} \mathrm{Hg}_{\nu}$. With high confidence we can expect $j^{(\nu)}$ for $\nu=13,15,17$ to describe a subfactor of Izumi type $\mathbb{Z}_{\nu}$ realising the modular data $\mathcal{D}^{0} \mathrm{Hg}_{\nu}$.

For $\nu=11,19$ respectively, $\mu=5^{3}, 5 \cdot 73$ are composite and the situation is more subtle. We find (up to the numerical accuracy of Maple) that for these $j^{(\nu)}$, $\omega_{l}=\xi_{\mu}^{m l^{2}}$ in (4.6) yields the correct multiplicity of solutions, and thus the corresponding $T$ matrix agrees with that of $\mathcal{D}^{0} \mathrm{Hg}_{\nu}$. Likewise, the solution $j^{(19) \prime}$ corresponds to $\mathcal{D}^{0,\{5,73\}} \mathrm{Hg}_{19}$. As with $\nu=9$, the existence of higher multiplicities means we cannot determine $S$ unambiguously from (4.7) for these $3 A$ s. We expect $j^{(11)}, j^{(19)}, j^{(19) \prime}$ to correspond to actual solutions of (4.1)-(4.3), hence subfactors of Izumi type $\mathbb{Z}_{11}, \mathbb{Z}_{19}, \mathbb{Z}_{19}$ respectively, with modular data $\mathcal{D}^{0} \mathrm{Hg}_{11}, \mathcal{D}^{0} \mathrm{Hg}_{19}, \mathcal{D}^{0,\{5,73\}} \mathrm{Hg}_{19}$ resp. 
The only unexpected solution here is $j^{(11) \prime}$. Following the method described in the proof of Theorem 5, we obtain the corresponding $T$ matrix: for each $1 \leq l \leq m$,

$$
T_{\mathfrak{d}_{l}, \mathfrak{d}_{l}}=\left\{\begin{array}{cc}
\xi_{\mu}^{m l^{2}} & \text { if } 5 \mid l \\
\xi_{\mu}^{5 m l^{2}} & \text { otherwise }
\end{array}\right.
$$

As before, $S$ is not uniquely determined by (4.7). We do not have a guess for what this $S$ matrix is, though its entries $S_{\mathfrak{v}_{l}, \mathfrak{o}^{\prime}}$ should lie in $\mathbb{Q}[\cos (2 \pi / 25)]$. Just as extra solutions like $j^{(9) \prime}, j^{(19) \prime}$ occur whenever distinct primes divide $\mu$, we would expect that solutions like $j^{(11) \prime}$ occur whenever a nontrivial prime power divides $\mu$.

On the basis of these observations, it is tempting to make the following conjecture.

Conjecture 1. The modular data $\mathcal{D}^{0, P} \mathrm{Hg}_{\nu}$ for any $\nu=2 n+1$ and any set $P$ (possibly empty) consisting of an even number of distinct prime divisors of $\mu$, is realised by the even part of the quantum double of a subfactor of Izumi type $\mathbb{Z}_{\nu}$. All $A \in \mathcal{A}(K)$ should correspond to modular data obeying $T^{2 \mu \nu}=I$.

We expect $T_{\mathfrak{d}_{l}, \mathfrak{o}_{l}}^{2 \mu}=1$ for reasons clear from the proof of Theorem 5 . Existence is accumulating suggesting that (up to equivalence) there is a unique matrix $A \in \mathcal{A}\left(\mathbb{Z}_{\nu}\right)$, and a unique subfactor of Izumi type $\mathbb{Z}_{\nu}$, iff $\mu=\nu^{2}+4$ is prime. It would be interesting to see if $\mathcal{A}(K)$ can be nonempty when $K$ is not cyclic.

Question 5. Interpret the $\mathbb{Z}_{\nu}$ twist in $\mathcal{D}^{\omega} \mathrm{Hg}_{\nu}$ in a von Neumann algebra formalism.

We would expect this to be in terms of twisted systems of endomorphisms, or twisted fusion categories, as what happens for twisted doubles of finite groups.

\subsection{Principal graphs, $\alpha$-induction etc for Izumi's subfactors}

Consider a subfactor $N \subset M$ of Izumi type $K$, an abelian group of odd order $\nu$, defined by some matrix $A \in \mathcal{A}(K)$, with $N-N$ and $M-M$ systems $\Delta$ and $\widehat{\Delta}$ respectively. We argued last subsection that the modular data of the double $\mathcal{D} \Delta$ and $\mathcal{D} \widehat{\Delta}$ is often (always?) the untwisted Haagerup-Izumi modular data $\mathcal{D}^{0} \mathrm{Hg}_{\nu}$ or some generalisation thereof. This subsection describes the data associated to this subfactor, generalising Section 2.2. For the special case of the Haagerup subfactor, i.e. $K=\mathbb{Z}_{3}$, much of this was computed in [5], though without appreciating the underlying $\mathbb{Z}_{3}$ structure which organises everything.

Write $\kappa$ for the inclusion $N \subset M$. Write $K^{\prime}$ for a set of representatives of the equivalence classes $(K \backslash 0) / \pm$; then $g \in K^{\prime}$ labels the conjugacy classes $|g|:=\{g,-g\}$ in the dihedral group $K \rtimes \mathbb{Z}_{2}$ (where $\mathbb{Z}_{2}$ acts by inverse). Recall $\delta=(\nu+\sqrt{\mu}) / 2$, $n=(\nu-1) / 2, m=\left(\nu^{2}+3\right) / 2, \mu=\nu^{2}+4=2 m+1$ and write $\lambda=\sqrt{1+\delta}$. 


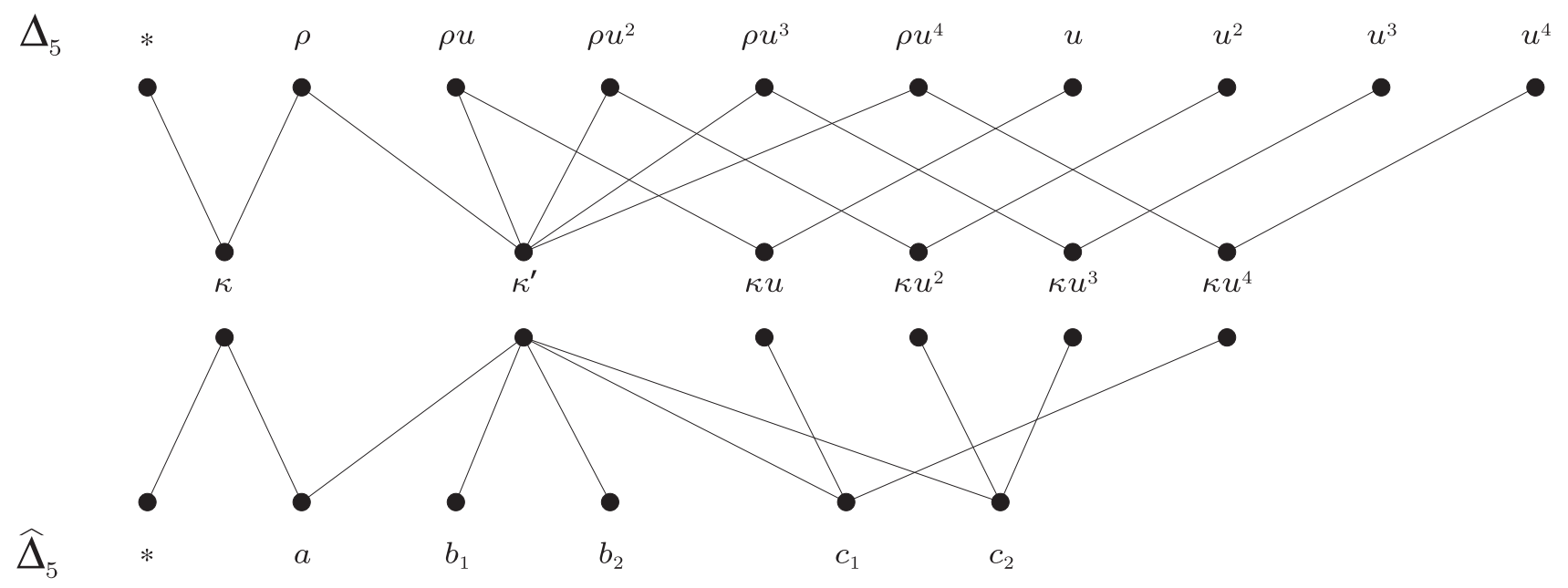

Figure 4. Principal graphs for Izumi's $\mathbb{Z}_{5}$ subfactor

Theorem 6. Let $N \subset M$ be a subfactor of Izumi type $K$ as above. It has index $\delta+1$. The $N-N$ system is $\Delta=\left\{\mathrm{id}, u, \ldots, u_{\nu-1}, \rho, \rho u, \ldots, \rho u_{\nu-1}\right\}$, and its sectors have fusions generated by

$$
\left[u_{g}\right]\left[u_{h}\right]=\left[u_{g+h}\right],\left[u_{g}\right][\rho]=[\rho]\left[u_{-g}\right],[\rho]^{2}=[\mathrm{id}]+\sum\left[\rho u_{g}\right] .
$$

The $M-N$ system consists of endomorphisms $\kappa u_{g}(g \in K)$ and $\kappa^{\prime}$. The principal graph consists of $\nu$ segments, each of length 3, sharing a common central vertex. The product $M-N \times N-N \rightarrow M-N$ is

$$
\begin{aligned}
& {\left[\kappa u_{g}\right]\left[\rho u_{h}\right]=\left[\kappa^{\prime}\right]+\left[\kappa u_{-g+h}\right],\left[\kappa^{\prime}\right]\left[\rho u_{g}\right]=\sum\left[\kappa u_{h}\right]+(\nu-1)\left[\kappa^{\prime}\right],} \\
& {\left[\kappa u_{g}\right]\left[u_{h}\right]=\left[\kappa u_{g+h}\right],\left[\kappa^{\prime}\right]\left[u_{g}\right]=\left[\kappa^{\prime}\right] .}
\end{aligned}
$$

The product $N-M \times M-N \rightarrow N-N$ is:

$$
\begin{aligned}
& {\left[\overline{\kappa u_{g}}\right]\left[\kappa u_{h}\right]=\left[u_{h-g}\right]+\left[\rho u_{h+g}\right],\left[\overline{\kappa^{\prime}}\right]\left[\kappa u_{g}\right]=\sum\left[\rho u_{h}\right],} \\
& {\left[\overline{\kappa^{\prime}}\right]\left[\kappa^{\prime}\right]=\sum\left[u_{g}\right]+(\nu-1) \sum\left[\rho u_{g}\right] .}
\end{aligned}
$$

The primaries of the double $\mathcal{D} \Delta$ are $0, \mathfrak{b}, \mathfrak{a}_{i}, \mathfrak{c}_{i, j}, \mathfrak{d}_{l}$ for $i \in K^{\prime}, j \in K, 1 \leq l \leq m$. The canonical endomorphism is $\theta_{\mathcal{D} \Delta}=1+\mathfrak{b}+2 \sum_{i} \mathfrak{a}_{i}$ with modular invariant $Z_{\Delta}=$ $\left|c h_{0}+c h_{\mathfrak{b}}+2 \sum_{i} c h_{\mathfrak{a}_{i}}\right|^{2}$. The alpha-inductions are $\alpha_{x}^{+}=\alpha x \otimes 1, \alpha_{x}^{-}=1 \otimes(\alpha x)^{\mathrm{opp}}$ where, for all $i \in K^{\prime}, j \in K, 1 \leq l \leq m$,

$$
\begin{array}{r}
{\left[\alpha \mathfrak{a}_{i}\right]=2[\mathrm{id}]+\sum\left[\rho u_{j}\right],[\alpha \mathfrak{b}]=[\mathrm{id}]+\sum\left[\rho u_{j}\right],} \\
{\left[\alpha \mathfrak{c}_{i, j}\right]=\left[u_{i}\right]+\left[u_{-i}\right]+\sum\left[\rho u_{j^{\prime}}\right], \quad\left[\alpha \mathfrak{d}_{l}\right]=\sum\left[\rho u_{j}\right] .}
\end{array}
$$

Proof. In Lemma 7.1 and Theorem 7.2 of [38], we learn that $\Delta$ contains $u_{g}$ and $\rho u_{g}$ for all $g \in K$, and the fusions $[\bar{\kappa} \kappa]=[\mathrm{id}]+[\rho],\left[u_{g}\right]\left[u_{h}\right]=\left[u_{g+h}\right]$ and $[\rho]^{2}=[\mathrm{id}]+\sum\left[\rho u_{g}\right]$. 
By definition $\Delta$ consists of all irreducible sectors in any $(\bar{\kappa} \kappa)^{k}$, and from this we quickly obtain that $\Delta=\left\{u_{g}, \rho u_{g}\right\}$. Because $\langle\kappa \rho, \kappa \rho\rangle=\langle\bar{\kappa} \kappa \rho, \rho\rangle=\langle(\rho+1) \rho, \rho\rangle=2$ and $\left\langle\kappa \rho, \kappa u_{g}\right\rangle=\left\langle(\rho+1) \rho, u_{g}\right\rangle=\delta_{g, 0}$, we see that $[\kappa \rho]-[\kappa]:=\left[\kappa^{\prime}\right]$ is an irreducible $M-N$ sector distinct from any $\left[\kappa u_{g}\right]$. We obtain the fusion $\left[\kappa^{\prime} u_{g}\right]=\left[\kappa^{\prime}\right]$ from the calculation $\left\langle\kappa^{\prime} u_{g}, \kappa^{\prime}\right\rangle=\left\langle\kappa(\rho-1) u_{g}, \kappa(\rho-1)\right\rangle=\left\langle\left(\rho^{2}-1\right) u_{g}, \rho-1\right\rangle=1$. $[\bar{\kappa}]\left[\kappa^{\prime}\right]$ has dimension $\lambda^{2}(\delta-1)=\nu \delta$ so will equal the sum of $\nu$ sectors $\left[\rho u_{g}\right]$, perhaps with multiplicities, but $K$-invariance now forces $[\bar{\kappa}]\left[\kappa^{\prime}\right]=\sum\left[\rho u_{g}\right]$. As above we see that the only sectors in $\kappa(\bar{\kappa} \kappa)^{k}$ are $\left[\rho u_{g}\right],\left[\kappa^{\prime}\right]$, so we have exhausted all $M-N$ sectors, and obtain the given principal graph. All remaining products are now easy to compute. For example, $\overline{\kappa^{\prime}} \kappa^{\prime}$ has dimension $\lambda^{2}(\delta-1)^{2}=\nu+(\nu-1) \nu \delta$, so by $K$-invariance this must be $\sum\left[u_{g}\right]+(\nu-1) \sum\left[\rho u_{g}\right]$.

At the end of Section 4 of [37] is explained how to recover the induction-restriction (i.e. dual principal) graph between $A-B$ and $A-A$ sectors, from the structure of Tube $(\Delta)$. In particular, we obtain the dual principal graph directly from the halfbraidings as described in Proposition 8.2 of [38] - for instance $\theta$ is read off from the half-braidings containing the identity. From the graph we read off the given alpha-inductions. QED

From this the dual principal graph for the double $\mathcal{D} \Delta$ is the obvious analogue of the top graph of Figure 3. Note the resemblance between the fusions for $\Delta$ and the group algebra $\mathbb{C} D_{K}$ of $D_{K}=K \rtimes \mathbb{Z}_{2}$, where $\mathbb{Z}_{2}$ acts on $K$ by inverse. We also see that the sectors $\left[u_{g}\right],\left[\rho u_{g}\right],\left[\kappa u_{g}\right]$, and $\left[\kappa^{\prime}\right]$ have statistical dimensions $1, \delta, \lambda$ and $(\delta-1) \lambda$ respectively. It is far from obvious (though of course an immediate consequence of Theorem 6) that the map $\alpha$ defined there is a ring homomorphism.

Theorem 7. Assume the hypotheses and notation of Theorem 6. In addition, assume the fusions of the double system $\mathcal{D} \Delta=\left\{\mathrm{id}, \mathfrak{b}, \mathfrak{a}_{i}, \mathfrak{c}_{i, j}, \mathfrak{d}_{l}\right\}$ agree with those of $\mathcal{D}^{0} \mathrm{Hg}_{\nu}$, and that we have the canonical endomorphism $\theta_{\mathcal{D} \widehat{\Delta}}=1+\mathfrak{b}+\sum \mathfrak{a}_{i}+\sum \mathfrak{c}_{h, 0}$. Then we can identify the $M-M$ system $\widehat{\Delta}$ with $\left\{\mathrm{id}, a, b_{i}, c_{i}\right\}_{i \in K^{\prime}}$ where $c_{i}=\kappa u_{i} \bar{\kappa} \cong \kappa u_{-i} \bar{\kappa}$. Its sectors obey the fusions

$$
\begin{array}{r}
{[a]^{2}=[\mathrm{id}]+[a]+\sum\left[b_{i}\right]+\sum\left[c_{i}\right], \quad[a]\left[b_{i}\right]=[a]+\sum\left[b_{k}\right]-\left[b_{i}\right]+\sum\left[c_{k}\right],} \\
{[a]\left[c_{i}\right]=[a]+\sum\left[b_{k}\right]+\left[c_{i}\right]+\sum\left[c_{k}\right], \quad\left[b_{i}\right]\left[c_{j}\right]=[a]+\sum\left[b_{k}\right]+\sum\left[c_{k}\right],} \\
{\left[b_{i}\right]\left[b_{j}\right]=\delta_{i, j}[\mathrm{id}]+\left(1-\delta_{i, j}\right)[a]+\sum\left[b_{k}\right]-\left[b_{i+j}\right]-\left[b_{i-j}\right]+\sum\left[c_{k}\right],} \\
{\left[c_{i}\right]\left[c_{j}\right]=\delta_{i, j}[\mathrm{id}]+\left(1+\delta_{i, j}\right)[a]+\sum\left[b_{k}\right]+\left[c_{i+j}\right]+\left[c_{i-j}\right]+\sum\left[c_{k}\right],}
\end{array}
$$

where we set $\left[c_{0}\right]=0=\left[b_{0}\right]$ and write $\left[c_{-i}\right]=\left[c_{i}\right],\left[b_{-i}\right]=\left[b_{i}\right]$. The dual principal graph is as in Figure 4: e.g. there is a (odd) central vertex, to which is attached $[a]$, the $\left[b_{i}\right]^{\prime}$ 's and the $\left[c_{i}\right]^{\prime}$ 's. The product $M-M \times M-N \rightarrow M-N$ is

$$
\begin{aligned}
& {[a]\left[\kappa u_{g}\right]=\left[\kappa u_{g}\right]+\left[\kappa^{\prime}\right],[a]\left[\kappa^{\prime}\right]=\sum\left[\kappa u_{g}\right]+(\nu-1)\left[\kappa^{\prime}\right], \quad\left[b_{i}\right]\left[\kappa u_{g}\right]=\left[\kappa^{\prime}\right],} \\
& {\left[b_{i}\right]\left[\kappa^{\prime}\right]=(\nu-2)\left[\kappa^{\prime}\right]+\sum\left[\kappa u_{g}\right], \quad\left[c_{i}\right]\left[\kappa u_{g}\right]=\left[u_{g+i}\right]+\left[\kappa u_{g-i}\right]+\left[\kappa^{\prime}\right],} \\
& {\left[c_{i}\right]\left[\kappa^{\prime}\right]=\sum\left[\kappa u_{g}\right]+\nu\left[\kappa^{\prime}\right],}
\end{aligned}
$$


while the product $M-N \times N-M \rightarrow M-M$ is

$$
\begin{aligned}
& {\left[\kappa u_{g}\right]\left[\overline{\kappa u_{h}}\right]=([\mathrm{id}]+[a]) \delta_{g, h}+\left[c_{g-h}\right]\left(1-\delta_{g, h}\right),\left[\kappa u_{g}\right]\left[\overline{\kappa^{\prime}}\right]=[a]+\sum\left[b_{i}\right]+\sum\left[c_{i}\right],} \\
& {\left[\kappa^{\prime}\right]\left[\overline{\kappa^{\prime}}\right]=[\mathrm{id}]+(\nu-1)[a]+(\nu-2) \sum\left[b_{i}\right]+\nu \sum\left[c_{i}\right] .}
\end{aligned}
$$

The dual canonical modular invariant is $\widehat{Z}=\left|c h_{0}+c h_{\mathfrak{b}}+\sum c h_{\mathfrak{a}_{i}}+\sum c h_{\mathfrak{c}_{h, 0}}\right|^{2}$. Writing $\widehat{\alpha}_{x}^{+}=\widehat{\alpha} x \otimes 1$ and $\widehat{\alpha}_{x}^{-}=1 \otimes(\widehat{\alpha} x)^{\mathrm{opp}}$, the alpha-inductions are

$$
\begin{aligned}
& {\left[\widehat{\alpha} \mathfrak{a}_{i}\right]=[\mathrm{id}]+2[a]+\sum\left[b_{h}\right]-\left[b_{i}\right]+\sum\left[c_{h}\right],[\widehat{\alpha} \mathfrak{b}]=[\mathrm{id}]+[a]+\sum\left[b_{h}\right]+\sum\left[c_{h}\right]} \\
& {\left[\widehat{\alpha} \mathfrak{c}_{i, j}\right]=[\mathrm{id}] \delta_{j, 0}+\left([a]-\left[b_{i}\right]\right)\left(1-\delta_{j, 0}\right)+\sum\left[b_{h}\right]+\left[c_{i}\right]+\sum\left[c_{h}\right]} \\
& {\left[\widehat{\alpha} \mathfrak{d}_{l}\right]=[a]+\sum\left[b_{i}\right]+\sum\left[c_{i}\right] .}
\end{aligned}
$$

Proof. From $\widehat{\theta}$ we obtain immediately $\widehat{Z}$. The cardinality of $\widehat{\Delta}$ is determined from $\langle\widehat{\gamma}, \widehat{\gamma}\rangle=\langle\widehat{\theta}, \widehat{\theta}\rangle=\sum \widehat{Z}_{i, 0}^{2}=\nu+1$. Fix any $l$ and define $[\mathrm{id}]=\left[\widehat{\alpha}_{0}\right]$ (the identity sector of $\widehat{\Delta}), A=\left[\widehat{\alpha}\left(\mathfrak{d}_{l}-\sum \mathfrak{c}_{h, 0}+\sum \mathfrak{a}_{i}\right)\right], B_{i}=A+\nu\left[\widehat{\alpha}\left(\mathfrak{b}-\mathfrak{a}_{i}\right)\right], C_{h}=A+\nu\left[\widehat{\alpha}\left(\widehat{c}_{h, 0}-\widehat{b}\right)\right]$. We compute (using $\langle\widehat{\alpha} x, \widehat{\alpha} y\rangle=\langle\widehat{\theta}, x y\rangle$ and the fusions of the double computed in Section 3.2) that $\left\langle\widehat{\alpha}\left(\mathfrak{a}_{i}-\mathfrak{b}\right), \widehat{\alpha}\left(\mathfrak{a}_{i^{\prime}}-\mathfrak{b}\right)\right\rangle=1+\delta_{i, i^{\prime}},\left\langle\mathfrak{c}_{h}-\mathfrak{b}, \mathfrak{c}_{h^{\prime}}-\mathfrak{b}\right\rangle=1+\delta_{h, h^{\prime}}$, $\left\langle\mathfrak{a}_{i}-\mathfrak{b}, \mathfrak{c}_{h}-\mathfrak{b}\right\rangle=-1,\left\langle\mathfrak{d}_{l}, \mathfrak{a}_{i}-\mathfrak{b}\right\rangle=\left\langle\mathfrak{d}_{l}, \mathfrak{c}_{h}-\mathfrak{b}\right\rangle=0,\left\langle\mathfrak{d}_{l}, \mathfrak{d}_{l}\right\rangle=\nu$. Hence the $A, B_{i}$, $C_{h}$ are mutually orthogonal, each with norm $\nu$, so they form a basis (over $\mathbb{Q}$ ) for the vector space spanned by the sectors of $\widehat{\Delta}$.

Let $\left[e_{k}\right]_{0 \leq k \leq \nu}$ be the irreducible sectors of $\widehat{\Delta}$; choose $\left[e_{0}\right]=[\mathrm{id}]$. Then $[A],\left[B_{i}\right],\left[C_{i}\right]$ are linear combinations (over $\mathbb{Z}$ ) of these $\left[e_{k}\right]$ : define $\vec{A}, \vec{L}^{(1)}, \ldots, \vec{L}^{(2 n)} \in \mathbb{Z}^{\nu+1}$ by $[A]=\sum \vec{A}_{k}\left[e_{k}\right],\left[B_{i}\right]=[A]+\nu \sum \vec{L}_{k}{ }^{(i)}\left[e_{k}\right],\left[C_{i}\right]=[A]+\nu \sum \vec{L}_{k}{ }^{(i+n)}\left[e_{k}\right]$. Then $\vec{A} \cdot \vec{A}=$ $\nu^{2}, \vec{A} \cdot \vec{L}^{(k)}=-\nu, \vec{L}^{(k)} \cdot \vec{L}^{(l)}=1+\delta_{k, l}$. From $\left\langle[\mathrm{id}], \widehat{\alpha}_{\mathfrak{a}_{i}}-\widehat{\alpha}_{\mathfrak{b}}\right\rangle=\left\langle[\mathrm{id}], \widehat{\alpha}_{\mathfrak{c}_{h, 0}}-\widehat{\alpha}_{\mathfrak{b}}\right\rangle=$ $\left\langle[\mathrm{id}], \widehat{\alpha}_{\mathfrak{D}_{l}}\right\rangle=0$ we have $\vec{A}_{0}=\vec{L}_{0}^{(k)}=0$. Thus (reordering the [e $e_{k}$ if necessary) $\vec{A}=$ $\left(0, s_{1}(\nu+t), \ldots, s_{\nu}(\nu+t), s t\right)$ and $\vec{L}^{(k)}$ has components $\vec{L}_{i}^{(k)}=s \delta_{i, \nu}+s_{k} \delta_{i, k}$, where $s, s_{k} \in\{ \pm 1\}$ and $t \in \mathbb{Z}$. This integer $t$ satisfies $\nu^{2}=t^{2}+\nu(\nu+t)^{2}$, so $t=-\nu$.

This means that $[a]:=\frac{1}{\nu}[A]$, and hence $\left[b_{i}\right]:=\frac{1}{\nu}\left[B_{i}\right]$ and $\left[c_{i}\right]:=\frac{1}{\nu}\left[C_{i}\right]$, all being norm 1 , are in $\widehat{\Delta}$, possibly up to signs. That the signs are indeed all +1 's follows by writing say $\left[\widehat{\alpha} \mathfrak{d}_{1}\right]$ in terms of $[a],\left[b_{i}\right],\left[c_{i}\right]$ : the coefficients should be nonnegative.

The fusions in $\widehat{\Delta}$ now reduce to the fusions in the double. The dual principal graph and the two remaining products follow by arguments as in Theorem 6. QED

This guess for $\widehat{\theta}$ is the natural one, matching what happens for the Haagerup subfactor (recall Section 2.2) and what we know about monomial modular invariants in Proposition 4, which appear to be severely constrained. The only example we know violating the hypothesis on the fusions of $\mathcal{D} \Delta$ is the solution $j^{(11) \prime}$ of Section 4.1 . The noncommutativity of $\Delta$ and commutativity of $\widehat{\Delta}$ arise because of the presence respectively absence of higher multiplicities in $\theta$ resp. $\widehat{\theta}$. The generalisation of Figure 3 can be read off from the alpha-inductions listed in the Theorem. These imply the statistical dimensions $\operatorname{dim}(a)=\delta, \operatorname{dim}\left(b_{i}\right)=\delta-1$ and $\operatorname{dim}\left(c_{j}\right)=\delta+1$. 
The dihedral group $K \rtimes \mathbb{Z}_{2}$ has one nontrivial 1-dimensional irrep and $n$ 2dimensional irreps. But for the Haagerup considered in Section 2.2, $a$ corresponded to the 2-dimensional and $b$ the 1-dimensional irreps, so $\widehat{K \rtimes \mathbb{Z}}_{2}$ and $\widehat{\Delta}$ here are unrelated for $\nu>3$, even after projecting away the $c_{i}$ 's.

\section{VOAs for Haagerup-Izumi modular data}

\subsection{The Haagerup-dihedral diamond}

In Section 2.4 we saw $\mathcal{D H g}$ as a mutation of $\mathcal{D} S_{3}$ and contained in a conformal subalgebra of $\mathcal{D} \mathbb{Z}_{3}$; now this becomes $\mathcal{D}^{\omega} \mathrm{Hg}_{\nu}$ is a mutation of $\mathcal{D}^{+, \omega} D_{\nu}$ and is contained in a conformal subalgebra of $\mathcal{D}^{\omega} \mathbb{Z}_{\nu}$. The branching rules for this embedding can be read off from the modular invariant (3.11). In some ways (e.g. modular data) the Haagerup-Izumi closely resembles its sibling the dihedral group, and in other ways (e.g. VOA realisations) it more closely relates to its parent the cyclic group.

The trivial case $\mathcal{D}^{0} \mathrm{Hg}_{1}$ is already interesting. In $c=8$, its VOA can be realised as the tensor of the affine algebra VOA $\mathcal{V}\left(G_{2,1}\right)$ (which has $c=14 / 5$ ), with the affine algebra VOA $\mathcal{V}\left(F_{4,1}\right)$ (which has $c=26 / 5$ ). (Hence there is a VOA realisation for $c$ any positive multiple of 8 , by tensoring by copies of $\mathcal{V}\left(E_{8}\right)$.) Call this $\mathcal{V}\left(\operatorname{Hg}_{1}^{0}\right)$. The VOA corresponding to $\mathcal{D} \mathbb{Z}_{1}$ in $c=8$ of course must be $\mathcal{V}\left(E_{8}\right)=\mathcal{V}\left(E_{8,1}\right)$, and the containment corresponds to the conformal embedding $G_{2,1} F_{4,1} \subset E_{8,1}$. The VOA corresponding to $\mathcal{V} D_{1}$ is the lattice $\operatorname{VOA} \mathcal{V}\left(A_{1} E_{7}\right)$. Although the latter is an orbifold of $\mathcal{V}\left(E_{8}\right), \mathcal{V}\left(G_{2,1} F_{4,1}\right)$ cannot be, since its modular data is not that of the (possibly twisted) quantum double of a finite group. This is an important lesson: we cannot expect the $\mathcal{D}^{\omega} \mathrm{Hg}_{\nu}$ VOAs to be orbifolds, by some subgroup of automorphisms, of $\mathcal{D}^{\omega} \mathbb{Z}_{\nu}$ VOAs. Hence Question 3.

Another important observation from this trivial case $\nu=1$ is that the intersection of the holomorphic orbifold $\mathcal{V} D_{1}$ with the $\nu=1$ Haagerup-Izumi VOA $\mathcal{V}\left(\mathrm{Hg}_{1}^{0}\right)$ is itself a rational VOA. In particular, there are conformal embeddings $\mathcal{V}\left(B_{3,1} G_{2,1}\right) \subset$ $\mathcal{V}\left(E_{7,1}\right)$ and $\mathcal{V}\left(A_{1,1} B_{3,1}\right) \subset \mathcal{V}\left(F_{4,1}\right)$ [56], and so both $\mathcal{V} D_{1}=\mathcal{V}\left(A_{1} E_{7}\right)$ and $\mathcal{V}\left(\mathrm{Hg}_{1}^{0}\right)=$ $\mathcal{V}\left(G_{2,1} F_{4,1}\right)$ contain copies of the rational VOA $\mathcal{V}\left(A_{1,1} G_{2,1} B_{3,1}\right)$. Thus in this baby example at least, the dihedral and Haagerup-Izumi VOAs are commensurable in this sense. It is tempting to guess though that something similar happens for higher $\nu$. This is what we mean by the Haagerup-dihedral diamond: at the top is a VOA realising $\mathcal{D}^{\omega} \mathbb{Z}_{\nu}$, which contains as conformal subalgebras $\mathcal{D}^{+, \omega} D_{\nu}$ and $\mathcal{D}^{\omega} \mathrm{Hg}$, and these contain a common conformal subalgebra (the analogue of $\left.\mathcal{V}\left(A_{1,1} G_{2,1} B_{3,1}\right)\right)$.

Conjecture 2. There is a rational VOA realisation of each $\mathcal{D}^{\omega} \mathrm{Hg}_{\nu}$ for any sufficiently large central charge $c$ (c a multiple of 8).

As $\nu$ increases, we will not be able to realise all $\mathcal{D}^{\omega} \mathrm{Hg}_{\nu}$ - or even the $\mathcal{D}^{\omega} \mathbb{Z}_{\nu}$ - as conformal subalgebras of $\mathcal{V}\left(E_{8}\right)$. In other words, $c>8$ will sometimes be necessary. This conjecture is open even for $\nu=3$. To make it more plausible, and also to aid in the construction of the VOAs, in the following sections we supply all the information 
needed to identify the possible character vectors. We saw in Section 2.4 that the Haagerup VOA would see the holomorphic orbifold by $\mathbb{Z}_{3}$ more directly than that by $S_{3}$, so for this reason we also supply the relevant data for $\mathcal{D}^{\omega} \mathbb{Z}_{3}$.

We should repeat here the suggestion of Section 2.5 that a second approach to constructing VOAs realising $\mathcal{D}^{\omega} \mathrm{Hg}_{\nu}$ is to use the affine algebra $\mathcal{V}\left(B_{m, 2}\right)$ in the coset construction. For reasons of space, we haven't explored this in this paper.

\subsection{Character vectors for the untwisted Haagerup double}

In this section we determine the matrices $\Lambda$ and $\chi$ for the untwisted Haagerup modular data $\mathcal{D}^{0} \mathrm{Hg}_{3}$, at all allowed values of central charge $c$ (i.e. when $8 \mid c$ ). As explained in Section 1.4, from this all possible character vectors can be obtained. We illustrate this with $c=16$ and $c=24$ (for $c=8$ see Section 2.4).

\subsubsection{Untwisted Haagerup at central charge $c \equiv_{24} 16$}

We found $\Lambda, \chi$ for $\mathcal{D}^{0} \mathrm{Hg}_{3}$ at $c \equiv_{24} 8$ in Section 2.4. Changing the central charge by a multiple of 8 leaves $S$ unchanged but multiplies $T$ by some 3 rd root $\omega$ of 1 . At the end of Section 1.4 we explain how this affects $\Lambda, \chi$. Taking the primaries in the usual order $0, \mathfrak{b} ; \mathfrak{a} ; \mathfrak{c}_{0}, \mathfrak{c}_{1}, \mathfrak{c}_{2} ; \mathfrak{d}_{1}, \ldots, \mathfrak{d}_{6}$, we obtain for $c \equiv_{24} 16$

$$
\begin{gathered}
\Lambda=\operatorname{diag}(1 / 3,1 / 3 ; 1 / 3 ; 1 / 3,2 / 3,0 ; 31 / 39,7 / 39,19 / 39,28 / 39,-5 / 39,-2 / 39), \\
\chi=\left(\begin{array}{cccccccccccc}
17 & 155 & 162 & 162 & 27 & 729 & 13 & 286 & 65 & 13 & 1001 & 728 \\
155 & 17 & 162 & 162 & 27 & 729 & -13 & -286 & -65 & -13 & -1001 & -728 \\
162 & 162 & 334 & -162 & -27 & -729 & 0 & 0 & 0 & 0 & 0 & 0 \\
162 & 162 & -162 & 334 & -27 & -729 & 0 & 0 & 0 & 0 & 0 & 0 \\
1215 & 1215 & -1215 & -1215 & -76 & 17496 & 0 & 0 & 0 & 0 & 0 & 0 \\
9 & 9 & -9 & -9 & 6 & -12 & 0 & 0 & 0 & 0 & 0 & 0 \\
1925 & -1925 & 0 & 0 & 0 & 0 & -21 & -6250 & -125 & 50 & 51331 & 43175 \\
45 & -45 & 0 & 0 & 0 & 0 & -9 & 58 & 43 & 12 & -45 & -297 \\
374 & -374 & 0 & 0 & 0 & 0 & 0 & 1650 & 106 & -51 & -4250 & 3927 \\
1288 & -1288 & 0 & 0 & 0 & 0 & 23 & 5313 & -759 & 4 & 39721 & -15432 \\
1 & -1 & 0 & 0 & 0 & 0 & 1 & 0 & -1 & 1 & 3 & 1 \\
3 & -3 & 0 & 0 & 0 & 0 & 3 & -9 & 4 & -2 & 6 & 4
\end{array}\right) .
\end{gathered}
$$

From (1.24) the matrix $\Xi(\tau)$ is recursively computed. Any weakly holomorphic vector-valued modular function equals $\Xi \vec{P}(J)$ for some polynomial vector $\vec{P}$.

Specialise now to a VOA character vector at $c=16$. The corresponding polynomials $P_{i}(x)$ are heavily constrained. For one thing, the vacuum character $c h_{0}(\tau)$ starts like $1 q^{-2 / 3}+\cdots$ and all other characters $c h_{i}(\tau)$ start like $a_{i} q^{h_{i}-c / 24}+\cdots$ where $h_{i}>0$. This forces $\vec{P}=(1,0,0,0, e, 0, g, h, 0, j, 0,0)^{T}$ for constants $e, g, h, j$. So we've uniquely determined the hypothetical $c=16$ Haagerup character vector, once 
4 numbers (namely $e, g, h, j$ ) are specified. Its first few terms are

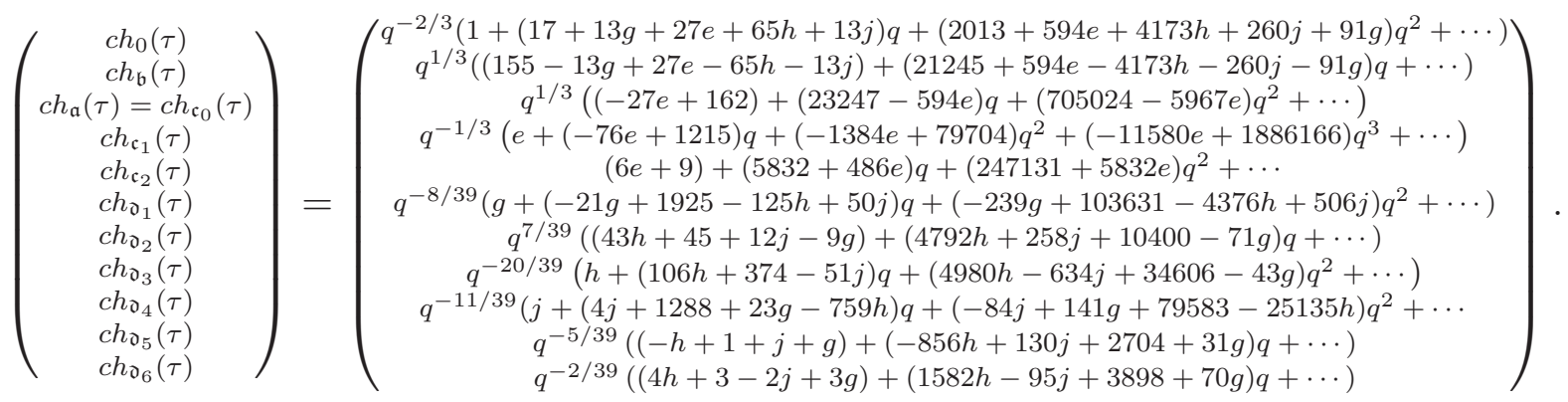

Since the Fourier coefficients of a VOA character vector are nonnegative integers, we know each $e, g, h, j \in \mathbb{Z}_{\geq 0}$. In addition the $q^{1 / 3}$-coefficient of $c h_{\mathfrak{a}}$ forces $e \leq 6$ and the $q^{1 / 3}$-coefficient of $c h_{\mathfrak{b}}$ then forces $g+j+5 h \leq 24$, so there are only finitely many possibilities. Other coefficients yield further bounds, e.g. $h \leq 2, j \leq 11, g \leq 16$.

There are several other constraints. One is that VOA characters are linear combinations over $\mathbb{Z}_{\geq 0}$ of the Virasoro characters at that $c$ for various values of conformal weight $h$ (because the VOA carries a Virasoro representation). All these characters are well-known: $\quad c h_{(16,0)}=q^{-2 / 3}\left(1+q^{2}+q^{3}+2 q^{4}+\cdots\right)$ and $\operatorname{ch}_{(24, h)}=q^{h-2 / 3}\left(1+q+2 q^{2}+3 q^{3}+5 q^{4}+\cdots\right)$ for $h>0$. This isn't so useful here, but it is effective for the $c=24$ case considered next.

More important, Dong-Mason [18] prove that the conformal weight 1 part of a rational VOA $\mathcal{V}$ is a reductive Lie algebra $\oplus_{i} \mathfrak{g}_{i}$ of central charge $\sum_{i} k_{i} \operatorname{dim} \mathfrak{g}_{i} /\left(k_{i}+\right.$ $\left.h_{i}^{\vee}\right) \leq c$ and dimension equal to the coefficient of $q^{1-c / 24}$ in $c h_{0}$. This gives a finite set of possible values for that coefficient, and knowing this reductive Lie algebra helps in constructing $\mathcal{V}$. Each $\mathcal{V}$-module is also a module for the corresponding affine Lie algebra, so the $\mathcal{V}$-characters are linear combinations of the affine characters.

This is as far as we'll carry the analysis here.

The matrices $\Lambda, \chi$ for $\mathcal{D}^{0} \mathbb{Z}_{3}$, with primaries in the order $(00),(01)=(02),(10)=$ $(20),(11)=(22),(12)=(21)$ are $\Lambda=\operatorname{diag}(1 / 3,1 / 3,1 / 3,2 / 3,0)$ and

$$
\chi=\left(\begin{array}{ccccc}
172 & 324 & 324 & 54 & 1458 \\
162 & 334 & -162 & -27 & -729 \\
162 & -162 & 334 & -27 & -729 \\
1215 & -1215 & -1215 & -76 & 17496 \\
9 & -9 & -9 & 6 & -12
\end{array}\right)
$$

At $c=16$ there is only one free parameter, $d$ : the possible character vectors are

$$
\left(\begin{array}{c}
\operatorname{ch}_{(0,0)} \\
\operatorname{ch}_{(0,1)}=\operatorname{ch}_{(0,2)}=\operatorname{ch}_{(1,0)} \\
\operatorname{ch}_{(1,1)}=\operatorname{ch}_{(2,2)} \\
\operatorname{ch}_{(1,2)}=\operatorname{ch}_{(2,1)}
\end{array}\right)=\left(\begin{array}{c}
q^{-2 / 3}\left(1+(172+54 d) q+(23258+1188 d) q^{2}+\cdots\right) \\
q^{1 / 3}\left((162-27 d)+(23247-594 d) q+(705024-5967 d) q^{2}+\cdots\right) \\
q^{-1 / 3}\left(d+(1215-76 d) q+(79704-1384 d) q^{2}+\cdots\right) \\
(9+6 d)+(5832+486 d) q+(247131+5832 d) q^{2}+\cdots
\end{array}\right)
$$

This implies $d \in \mathbb{Z}_{\geq 0}$ and $d \leq 6$. This $d$ equals the parameter $e$ of $\mathcal{D}^{0} \mathrm{Hg}$. 


\subsubsection{Untwisted Haagerup at central charge $c \equiv_{24} 0$}

Next turn to the Haagerup at $24 \mid c$. Finding its $\Lambda, \chi$ is now routine:

$$
\left.\chi=\begin{array}{cccccccccccc}
-12 & \multicolumn{1}{c}{(0,0,1,1,1 / 3,2 / 3,6 / 13,11 / 13,2 / 13,5 / 13,7 / 13,8 / 13),} \\
0 & 0 & 1 / 2 & 1 / 2 & 18 & 6 & 6 & 1 & 10 & 5 & 4 & 4 \\
65610 & 65610 & 1 / 2 & 1 / 2 & 18 & 6 & -6 & -1 & -10 & -5 & -4 & -4 \\
65610 & 65610 & 0 & 0 & -5832 & -243 & 0 & 0 & 0 & 0 & 0 & 0 \\
729 & 729 & 0 & 0 & -152 & -243 & 0 & 0 & 0 & 0 & 0 & 0 \\
8748 & 8748 & 0 & 0 & 2430 & -76 & 0 & 0 & 0 & 0 & 0 & 0 \\
1716 & -1716 & 0 & 0 & 0 & 0 & -252 & -5 & -176 & 176 & 134 & 100 \\
22451 & -22451 & 0 & 0 & 0 & 0 & -980 & 8 & 16556 & 2464 & -96 & -385 \\
104 & -104 & 0 & 0 & 0 & 0 & 0 & 5 & 44 & -56 & -15 & 20 \\
910 & -910 & 0 & 0 & 0 & 0 & 77 & 8 & -847 & -32 & 120 & -34 \\
3003 & -3003 & 0 & 0 & 0 & 0 & 330 & 0 & -1540 & 704 & -216 & 55 \\
5200 & -5200 & 0 & 0 & 0 & 0 & 616 & -14 & 3388 & -595 & 132 & -20
\end{array}\right) .
$$

Its possible character vectors are found as before to be

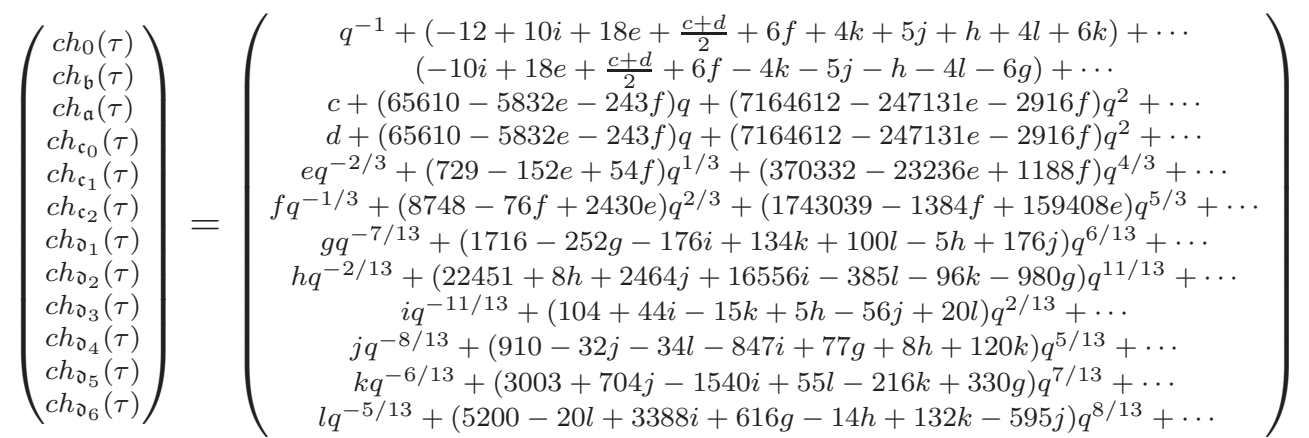

for certain nonnegative integers $c, d, e, f, g, h, i, j, k, l$. By the usual arguments we find a finite number of possibilities. Likewise the matrices for $\mathcal{D}^{0} \mathbb{Z}_{3}$ are $\Lambda=$ $\operatorname{diag}(0,1,1,1 / 3,2 / 3)$ and

$$
\chi=\left(\begin{array}{ccccc}
-12 & 1 & 1 & 36 & 12 \\
65610 & 0 & 0 & -5832 & -243 \\
65610 & 0 & 0 & -5832 & -243 \\
729 & 0 & 0 & -152 & 54 \\
8748 & 0 & 0 & 2430 & -76
\end{array}\right)
$$

and we find a character vector depending on 4 bounded nonnegative integers.

The most intriguing possibility is if these Haagerup and $\mathbb{Z}_{3}$ VOAs are both subVOAs of the Moonshine module. It is tempting to guess that this is the most natural home of the Haagerup. Then the character $J$ of the Moonshine module will equal one of $c h_{0}+c h_{\mathfrak{b}}+2 c h_{\mathfrak{a}}, c h_{0}+c h_{\mathfrak{b}}+2 c h_{\mathfrak{c}}$, or $c h_{0}+c h_{\mathfrak{b}}+c h_{\mathfrak{a}}+c h_{\mathfrak{c}_{0}}$, and we find that $(c, d, f)$ is either $(12,0,0),(0,12,0)$ or $(0,0,1)$, and that $(g, h, j, k, l)$ is either $(1,2,0,0,0)$, $(0,1,1,0,0),(0,2,0,1,0)$ or $(0,1,0,0,1)$, and all other parameters vanish.

\subsection{Character vectors of the twisted Haagerup double}

The modular group representation for the $\omega \neq 0$ twist of the Haagerup decomposes into a sum of 3 irreps: the trivial one, a 4-dimensional one with kernel $\Gamma(9)$, and 
a 7-dimensional one with kernel $\Gamma(13)$. Only the 4-dimensional irrep is new: it is handled by e.g. the root lattice $A_{8}$. Actually, 6 different 4-dimensional irreps arise here, varying with the twist and central charge mod 24 , but they are all obtained from the one in $A_{8}$ by some combination of 3rd roots of unity and taking the contragredient. The effects on $\Lambda, \chi$ of both of these is discussed at the end of Section 1.4.

\subsubsection{The 1-twisted Haagerup at central charge $c \equiv_{24} 8$}

The matrices $\Lambda, \chi$ for $\mathcal{D}^{1} \mathbb{Z}_{3}$ and $\mathcal{D}^{1} \mathrm{Hg}_{3}$ are respectively $\operatorname{diag}(2 / 3,2 / 3,7 / 9,1 / 9,4 / 9)$,

$\left(\begin{array}{ccccc}80 & 168 & 54 & 9504 & 1078 \\ 84 & 164 & -27 & -4752 & -539 \\ 126 & -126 & 8 & -17248 & 1375 \\ 9 & -9 & -8 & 96 & 10 \\ 36 & -36 & 20 & 392 & -340\end{array}\right)$,

$\operatorname{diag}(-1 / 3,2 / 3,2 / 3,7 / 9,1 / 9,4 / 9,5 / 39,20 / 39,32 / 39,41 / 39,8 / 39,11 / 39)$,

$\left(\begin{array}{cccccccccccc}-42 / 5 & 1 & 0 & 0 & 0 & 0 & -17 / 5 & -13 / 5 & 3 / 5 & 1 / 5 & -7 & 14 / 5 \\ 209456 / 5 & 80 & 84 & 81 & 14256 & 1620 & -19054 / 5 & -2366 / 5 & 166 / 5 & 77 / 5 & -2814 & -5812 / 5 \\ 228912 / 5 & 168 & 164 & -81 & -14256 & -1620 & -1428 / 5 & -1092 / 5 & 252 / 5 & 84 / 5 & -588 & 1176 / 5 \\ 176556 / 5 & 84 & -42 & 8 & -17248 & 1375 & -714 / 5 & -546 / 5 & 126 / 5 & 42 / 5 & -294 & 588 / 5 \\ 114 / 5 & 6 & -3 & -8 & 96 & 10 & -51 / 5 & -39 / 5 & 9 / 5 & 3 / 5 & -21 & 42 / 5 \\ 11391 / 5 & 24 & -12 & 20 & 392 & -340 & -204 / 5 & -156 / 5 & 36 / 5 & 12 / 5 & -84 & 168 / 5 \\ 546 / 5 & 0 & 0 & 0 & 0 & 0 & -559 / 5 & -161 / 5 & 16 / 5 & 7 / 5 & 14 & 418 / 5 \\ 52611 / 5 & 0 & 0 & 0 & 0 & 0 & -8134 / 5 & -86 / 5 & 206 / 5 & 42 / 5 & -486 & -4617 / 5 \\ 600457 / 5 & 0 & 0 & 0 & 0 & 0 & -12733 / 5 & 3388 / 5 & 412 / 5 & 119 / 5 & -6868 & 24871 / 5 \\ 2851862 / 5 & 0 & 0 & 0 & 0 & 0 & 100947 / 5 & 6118 / 5 & 437 / 5 & 229 / 5 & 31027 & -49514 / 5 \\ 1729 / 5 & 0 & 0 & 0 & 0 & 0 & 649 / 5 & -174 / 5 & 14 / 5 & 13 / 5 & -238 & 437 / 5 \\ 5252 / 5 & 0 & 0 & 0 & 0 & 0 & 2002 / 5 & -567 / 5 & 77 / 5 & 14 / 5 & 27 & 76 / 5\end{array}\right)$.

At $c=8, \mathcal{D}^{1} \mathbb{Z}_{3}$ is realised by the lattice $\operatorname{VOA} \mathcal{V}\left(A_{8}\right) . \mathcal{D}^{+, 1} S_{3}$ is realised by the affine algebra $\operatorname{VOA} \mathcal{V}\left(B_{4,2}\right)$. Both of these are conformal embeddings.

\subsubsection{The 1-twisted Haagerup at central charge $c \equiv_{24} 16$}

The matrices $\Lambda, \chi$ for $\mathcal{D}^{1} \mathbb{Z}_{3}$ and $\mathcal{D}^{1} \mathrm{Hg}_{3}$ are respectively $\operatorname{diag}(1 / 3,1 / 3,4 / 9,7 / 9,1 / 9)$,

$$
\chi=\left(\begin{array}{ccccc}
166 & 330 & 198 & 18 & 900 \\
165 & 331 & -99 & -9 & -450 \\
351 & -351 & 56 & -25 & 1625 \\
2079 & -2079 & -1694 & 53 & 3146 \\
27 & -27 & 28 & 1 & -102
\end{array}\right)
$$

$\operatorname{diag}(-2 / 3,1 / 3,1 / 3,4 / 9,7 / 9,1 / 9,31 / 39,7 / 39,19 / 39,28 / 39,34 / 39,-2 / 39)$,

$$
\left(\begin{array}{cccccccccccc}
3 & 1 & 0 & 0 & 0 & 0 & -1 & 0 & 1 & -1 & 1 & -1 \\
6732 & 166 & 165 & 297 & 27 & 1350 & -165 & -286 & 87 & -165 & 152 & -880 \\
7359 & 330 & 331 & -297 & -27 & -1350 & -165 & 0 & 165 & -165 & 165 & -165 \\
8199 & 234 & -117 & 56 & -25 & 1625 & -117 & 0 & 117 & -117 & 117 & -117 \\
173727 & 1386 & -693 & -1694 & 53 & 3146 & -693 & 0 & 693 & -693 & 693 & -693 \\
126 & 18 & -9 & 28 & 1 & -102 & -9 & 0 & 9 & -9 & 9 & -9 \\
498225 & 0 & 0 & 0 & 0 & 0 & -1946 & -6250 & 1800 & -1875 & 1925 & 41250 \\
858 & 0 & 0 & 0 & 0 & 0 & -54 & 58 & 88 & -33 & 45 & -342 \\
31603 & 0 & 0 & 0 & 0 & 0 & -374 & 1650 & 480 & -425 & 374 & 3553 \\
263120 & 0 & 0 & 0 & 0 & 0 & -1265 & 5313 & 529 & -1284 & 1288 & -16720 \\
920556 & 0 & 0 & 0 & 0 & 0 & -2673 & -2025 & 1848 & -2574 & 2704 & 22572 \\
13 & 0 & 0 & 0 & 0 & 0 & 0 & -9 & 7 & -5 & 3 & 1
\end{array}\right) .
$$




\subsubsection{The 1-twisted Haagerup at central charge $c \equiv_{24} 0$}

The matrices $\Lambda, \chi$ for $\mathcal{D}^{1} \mathbb{Z}_{3}$ and $\mathcal{D}^{1} \mathrm{Hg}_{3}$ are respectively $\operatorname{diag}(1,0,1 / 9,4 / 9,7 / 9)$,

$$
\left(\begin{array}{ccccc}
0 & 131274 & 185328 & 13770 & 324 \\
1 & 6 & -135 & -54 & -27 \\
0 & -27 & 24 & -35 & 10 \\
0 & -594 & -2002 & 290 & 11 \\
0 & -5967 & 14144 & 340 & -64
\end{array}\right)
$$

$\operatorname{diag}(0,1,0,1 / 9,4 / 9,7 / 9,6 / 13,11 / 13,2 / 13,5 / 13,7 / 13,8 / 13)$,

$$
\left(\begin{array}{cccccccccccc}
-12 & 1 & 0 & 0 & 0 & 0 & 20 & 2 & 10 & 8 & 12 & 8 \\
60033 & 0 & 65637 & 92664 & 6885 & 162 & -18997 & -16 & -3200 & -350 & -1617 & -792 \\
-18 & 2 & 6 & -135 & -54 & -27 & 20 & 2 & 10 & 8 & 12 & 8 \\
27 & 0 & -27 & 24 & -35 & 10 & 0 & 0 & 0 & 0 & 0 & 0 \\
594 & 0 & -594 & -2002 & 290 & 11 & 0 & 0 & 0 & 0 & 0 & 0 \\
5967 & 0 & -5967 & 14144 & 340 & -64 & 0 & 0 & 0 & 0 & 0 & 0 \\
1716 & 0 & 0 & 0 & 0 & 0 & -252 & -5 & -176 & 176 & 134 & 100 \\
22451 & 0 & 0 & 0 & 0 & 0 & -980 & 8 & 16556 & 2464 & -96 & -385 \\
104 & 0 & 0 & 0 & 0 & 0 & 0 & 5 & 44 & -56 & -15 & 20 \\
910 & 0 & 0 & 0 & 0 & 0 & 77 & 8 & -847 & -32 & 120 & -34 \\
3003 & 0 & 0 & 0 & 0 & 0 & 330 & 0 & -1540 & 704 & -216 & 55 \\
5200 & 0 & 0 & 0 & 0 & 0 & 616 & -14 & 3388 & -595 & 132 & -20
\end{array}\right) .
$$

\subsubsection{The 2-twisted Haagerup at central charge $c \equiv_{24} 8$}

The matrices $\Lambda, \chi$ for $\mathcal{D}^{2} \mathbb{Z}_{3}$ and $\mathcal{D}^{2} \mathrm{Hg}_{3}$ are respectively diag $(-1 / 3,2 / 3,8 / 9,2 / 9,5 / 9)$,

$$
\left(\begin{array}{ccccc}
2 & 1 & 1 / 3 & -1 / 3 & -2 / 3 \\
46683 & 248 & 26 & -836 & -133 \\
706401 & 0 & 156 & 2584 & 475 \\
2187 & 0 & 14 & 172 & -77 \\
56862 & 0 & 65 & -2431 & -74
\end{array}\right)
$$

$\operatorname{diag}(-1 / 3,-1 / 3,2 / 3,8 / 9,2 / 9,5 / 9,5 / 39,20 / 39,32 / 39,41 / 39,8 / 39,11 / 39)$,

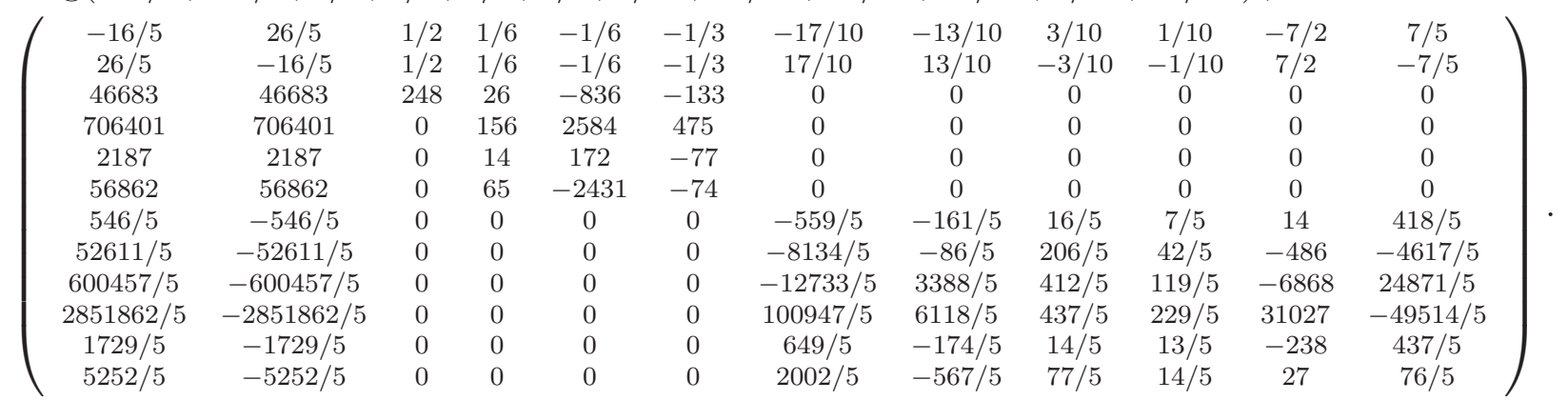




\subsubsection{The 2-twisted Haagerup at central charge $c \equiv_{24} 16$}

The matrices $\Lambda, \chi$ for $\mathcal{D}^{2} \mathbb{Z}_{3}$ and $\mathcal{D}^{2} \mathrm{Hg}_{3}$ are respectively $\operatorname{diag}(1 / 3,1 / 3,5 / 9,8 / 9,2 / 9)$,

$$
\left(\begin{array}{ccccc}
160 & 336 & 32 / 3 & 2 / 3 & 196 / 3 \\
168 & 328 & -16 / 3 & -1 / 3 & -98 / 3 \\
5832 & -5832 & -272 & 2 & 1925 \\
32076 & -32076 & 196 & 12 & -15092 \\
729 & -729 & 40 & -4 & 28
\end{array}\right),
$$

$\operatorname{diag}(-2 / 3,1 / 3,1 / 3,5 / 9,8 / 9,2 / 9,31 / 39,7 / 39,19 / 39,28 / 39,34 / 39,-2 / 39)$,

$$
\left(\begin{array}{cccccccccccc}
3 & 1 & 0 & 0 & 0 & 0 & -1 & 0 & 1 & -1 & 1 & -1 \\
6717 & 160 & 168 & 16 & 1 & 98 & -162 & -286 & 84 & -162 & 149 & -877 \\
7374 & 336 & 328 & -16 & -1 & -98 & -168 & 0 & 168 & -168 & 168 & -168 \\
221859 & 3888 & -1944 & -272 & 2 & 1925 & -1944 & 0 & 1944 & -1944 & 1944 & -1944 \\
3788856 & 21384 & -10692 & 196 & 12 & -15092 & -10692 & 0 & 10692 & -10692 & 10692 & -10692 \\
5589 & 486 & -243 & 40 & -4 & 28 & -243 & 0 & 243 & -243 & 243 & -243 \\
498225 & 0 & 0 & 0 & 0 & 0 & -1946 & -6250 & 1800 & -1875 & 1925 & 41250 \\
858 & 0 & 0 & 0 & 0 & 0 & -54 & 58 & 88 & -33 & 45 & -342 \\
31603 & 0 & 0 & 0 & 0 & 0 & -374 & 1650 & 480 & -425 & 374 & 3553 \\
263120 & 0 & 0 & 0 & 0 & 0 & -1265 & 5313 & 529 & -1284 & 1288 & -16720 \\
920556 & 0 & 0 & 0 & 0 & 0 & -2673 & -2025 & 1848 & -2574 & 2704 & 22572 \\
13 & 0 & 0 & 0 & 0 & 0 & 0 & -9 & 7 & -5 & 3 & 1
\end{array}\right) .
$$

\subsubsection{The 2-twisted Haagerup at central charge $c \equiv_{24} 0$}

The matrices $\Lambda, \chi$ for $\mathcal{D}^{2} \mathbb{Z}_{3}$ and $\mathcal{D}^{2} \mathrm{Hg}_{3}$ are respectively $\operatorname{diag}(1,0,8 / 9,5 / 9,2 / 9)$,

$$
\begin{aligned}
& \left(\begin{array}{ccccc}
0 & 131274 & 6 & 528 & 9282 \\
1 & -3 & -1 & -7 & -8 \\
0 & -104247 & 3 & -1001 & 12376 \\
0 & -12393 & -7 & 232 & 476 \\
0 & -729 & 5 & 22 & -224
\end{array}\right) \text {, } \\
& \operatorname{diag}(0,0,1,2 / 9,5 / 9,8 / 9,6 / 13,11 / 13,2 / 13,5 / 13,7 / 13,8 / 13) \text {, } \\
& \left(\begin{array}{cccccccccccc}
-15 / 2 & 9 / 2 & 1 / 2 & 4 & 7 / 2 & 1 / 2 & 6 & 1 & 10 & 5 & 4 & 4 \\
9 / 2 & -15 / 2 & 1 / 2 & 4 & 7 / 2 & 1 / 2 & -6 & -1 & -10 & -5 & -4 & -4 \\
65637 & 65637 & 0 & -4641 & -264 & -3 & 0 & 0 & 0 & 0 & 0 & 0 \\
729 & 729 & 0 & -224 & 22 & 5 & 0 & 0 & 0 & 0 & 0 & 0 \\
12393 & 12393 & 0 & 476 & 232 & -7 & 0 & 0 & 0 & 0 & 0 & 0 \\
104247 & 104247 & 0 & 12376 & -1001 & 3 & 0 & 0 & 0 & 0 & 0 & 0 \\
1716 & -1716 & 0 & 0 & 0 & 0 & -252 & -5 & -176 & 176 & 134 & 100 \\
22451 & -22451 & 0 & 0 & 0 & 0 & -980 & 8 & 16556 & 2464 & -96 & -385 \\
104 & -104 & 0 & 0 & 0 & 0 & 0 & 5 & 44 & -56 & -15 & 20 \\
910 & -910 & 0 & 0 & 0 & 0 & 77 & 8 & -847 & -32 & 120 & -34 \\
3003 & -3003 & 0 & 0 & 0 & 0 & 330 & 0 & -1540 & 704 & -216 & 55 \\
5200 & -5200 & 0 & 0 & 0 & 0 & 616 & -14 & 3388 & -595 & 132 & -20
\end{array}\right) .
\end{aligned}
$$

Acknowledgement.

The authors thank the University of Alberta Mathematics Dept, Cardiff School of Mathematics, Swansea University Dept of Computer Science, and Universität Würzburg Institut für Mathematik for generous hospitality while researching this paper. Their research was supported in part by EUNCG Research Training Network: MRTN-CT-2006 031962, DAAD (Prodi Chair), and NSERC. We thank Matthias Gaberdiel, Pinhas Grossman, Paulo Pinto, Ingo Runkel, and Feng Xu for discussions.

\section{References}

[1] Asaeda, M., Haagerup, U.: Exotic subfactors of finite depth with Jones indices $(5+\sqrt{13}) / 2$ and $(5+\sqrt{17}) / 2$. Commun. Math. Phys. 202, 1-63 (1999). 
[2] Bantay, P., Gannon, T.: Vector-valued modular functions for the modular group and the hypergeometric equation. Commun. Number Theory Phys. 1, 637-666 (2008).

[3] Bantay, P., Gannon, T.: On vector-valued modular forms. (in preparation).

[4] Bigelow, S., Morrison, S., Peters, E., Snyder, N.: Constructing the extended Haagerup planar algebra. arXiv: math.OA0909.4099.

[5] Bisch, D.: On the structure of finite depth subfactors. In: Algebraic methods in operator theory, 175-194, Birkhäuser Boston, Boston, MA, 1994.

[6] Böckenhauer, J., Evans, D.E.: Modular invariants, graphs and $\alpha$-induction for nets of subfactors, I. Commun. Math. Phys. 197, 361-386 (1998).

[7] Böckenhauer, J., Evans, D.E.: Modular invariants, graphs and $\alpha$-induction for nets of subfactors, III. Commun. Math. Phys. 205, 183-228 (1999).

[8] Böckenhauer, J., Evans, D.E.: Modular invariants from subfactors: Type I coupling matrices and intermediate subfactors. Commun. Math. Phys. 213, 267-289 (2000).

[9] Böckenhauer, J., Evans, D.E.: Modular invariants from subfactors. In: Quantum Symmetries in Theoretical Physics and Mathematics (Bariloche, 2000), pp. 95-131, Contemp. Math., 294, Amer. Math. Soc., Providence, RI, 2002.

[10] Böckenhauer, J., Evans, D.E.: Modular invariants and subfactors. In: Mathematical Physics in Mathematics and Physics (Siena, 2000), pp. 11-37, Fields Inst. Commun., 30, Amer. Math. Soc., 2001.

[11] Böckenhauer, J., Evans, D.E., Kawahigashi, Y.: On $\alpha$-induction, chiral generators and modular invariants for subfactors. Commun. Math. Phys. 208, 429-487 (1999).

[12] Böckenhauer, J., Evans, D.E., Kawahigashi, Y.: Chiral structure of modular invariants for subfactors. Commun. Math. Phys. 210, 733-784 (2000).

[13] Conway, J.H., Sloane, N.J.A.: Low-dimensional lattices. I: Quadratic forms of small determinant. Proc. R. Soc. Lond. A 418, 17-41 (1988).

[14] Coste, A., Gannon, T.: Remarks on Galois symmetry in rational conformal field theories. Phys. Lett. B323, 316-321 (1994).

[15] Coste, A., Gannon, T., Ruelle, P.: Finite group modular data. Nucl. Phys. B581, 679-717 (2000).

[16] Dijkgraaf, R., Vafa, C., Verlinde, E., Verlinde, H.: The operator algebra of orbifold models. Commun. Math. Phys. 123, 485-526 (1989).

[17] Dijkgraaf, R., Witten, E.: Topological gauge theories and group cohomology. Commun. Math. Phys. 129, 393-429 (1990).

[18] Dong, C., Mason, G.: Integrability of $C_{2}$-cofinite vertex operator algebras. arXiv: math/0601569

[19] Dovgard, R., Gepner, D.: Conformal field theories with a low number of primary fields. J. Phys. A, Math. Theor. 42, 304009, 8 pp. (2009).

[20] Evans, D. E.: Critical phenomena, modular invariants and operator algebras. In: Cuntz, J., Elliott, G. A., Stratila, S. et al (eds.) Operator algebras and mathematical physics. Proceedings, Constanţa 2001. Bucharest: The Theta Foundation, 2003, pp. 89-113.

[21] Evans, D.E.: From Ising to Haagerup. Markov Processes Relat. Fields 13, 267-287 (2007).

[22] Evans, D.E.: Twisted K-theory and modular invariants: I. Quantum doubles of finite groups. In: Bratteli, O., Neshveyev, S., Skau, C. (eds.) Operator Algebras: The Abel Symposium 2004. Berlin-Heidelberg: Springer, 2006, pp. 117-144.

[23] Evans, D. E., Gannon, T.: Modular invariants and twisted equivariant K-theory. Commun. Number Theory Phys. 3, 209-296 (2009).

[24] Evans, D.E., Kawahigashi, Y.: Orbifold subfactors from Hecke algebras II: quantum double and braiding. Commun. Math. Phys.196, 331-361 (1998); arXiv: funct-an/9702018

[25] Evans, D.E., Kawahigashi, Y.: Quantum Symmetries on Operator Algebras. Oxford University Press (1998).

[26] Evans, D.E., Pinto, P.R.: Subfactor realisation of modular invariants. Commun. Math. Phys. 237, 309-363 (2003).

[27] Evans, D.E. Pinto, P.R.: Modular invariants and the double of the Haagerup subfactor. In: Advances in Operator Algebras and Mathematical Physics (Sinaia 2003) The Theta Foundation, eds. F.-P. Boca, O. Bratteli, R. Longo, and H. Siedentop, Bucharest, 2006, pp.67-88.

[28] Evans, D.E., Pinto, P.R.: Subfactor realisation of modular invariants: II. (in preparation).

[29] Frenkel, I.B., Zhu, Y.: Vertex operator algebras associated to representations of affine and Virasoro algebras. Duke Math. J. 66, 123-168 (1992).

[30] Fröhlich, J., Fuchs, J., Runkel, I., Schweigert, C.: Defect lines, dualities, and generalised orbifolds. arXiv: math-ph 0909.5013.

[31] Gannon, T.: Modular data: the algebraic combinatorics of conformal field theory. J. Alg. Combin. 22, 211-250 (2005).

[32] Gannon, T.: The level 2 and 3 modular invariants for the orthogonal algebras. Canad. Math. J. 52, 503-521 (2000).

[33] Goddard, P., Kent, A., Olive, D.: Unitary representations of Virasoro and super-Virasoro algebras. Commun. Math. Phys. 103, 105-119 (1986). 
[34] Haagerup, U.: Principal graphs of subfactors in the index range $4<[M: N]<3+\sqrt{3}$. In: Subfactors, eds. H. Araki et al, World Scientific, 1994, pp.1-38.

[35] Hong, S.-M., Rowell, E., Wang, Z.: On exotic modular tensor categories. Commun. Contemp. Math. 10, Suppl. 1, 1049-1074 (2008).

[36] Huang, Y.-Z.: Vertex operator algebras, the Verlinde conjecture, and modular tensor categories. Proc. Natl. Acad. Sci. USA 102, 5352-5356 (2005).

[37] Izumi, M.: The structure of sectors associated with Longo-Rehren inclusions, I. General theory. Commun. Math. Phys. 213, 127-179 (2000).

[38] Izumi, M.: The structure of sectors associated with Longo-Rehren inclusions, II. Examples. Rev. Math. Phys. 13, 603-674 (2001).

[39] Izumi, M., Kawahigashi, Y.: Classification of subfactors with the principal graph $D_{n}^{(1)}$. J. Funct. Anal. 112, 257-286 (1993).

[40] Jones, V. F. R.: An invariant for group actions. In: de la Harpe, P. (ed.) Algèbres d'opèrateurs. Proceedings, Les Plans-sur-Bex 1978. Lecture Notes in Math. 725. Berlin: Springer, 1979, pp. 237-253.

[41] Jones, V.F.R.: Index for subfactors. Invent. Math. 72, 1-25 (1983).

[42] Kac, V.G.: Infinite-dimensional Lie algebras, 3rd edn. (Cambridge University Press, Cambridge 1990).

[43] Kac, V.G., Todorov, I.T.: Affine orbifolds and rational conformal field theory extensions of $W_{1+\infty}^{*}$. Commun. Math. Phys. 190, 57-111 (1997).

[44] Kac, V.G., Wakimoto, M.: Modular and conformal invariance constraints in representation theory of affine algebras. Adv. Math. 70, (1988) 156-236.

[45] Kawahigashi, Y., Longo, R., Müger, M.: Multi-interval subfactors and modularity of representations in conformal field theory. Commun. Math. Phys. 219, 631-669 (2001); arXiv: math.OA/9903104

[46] Kosaki, H., Munemasa, A., Yamagami, S.: Irreducible bimodules associated with crossed product algebras. Internat. J. Math. 3, 661-676 (1992).

[47] Lepowski, J., Li, H.: Introduction to Vertex Operator Algebras and their Representations. Boston, Birkhäuser, 2004.

[48] Longo, R.: Index of subfactors and statistics of quantum fields, I. Commun. Math. Phys. 126, 217-247 (1989).

[49] Longo, R., Rehren, K.-H.: Nets of subfactors. Rev. Math. Phys. 7, 567-597 (1995).

[50] Masuda, T.: An analogue of Longo's canonical endomorphism for bimodule theory and its application to asymptotic inclusions. Internat. J. Math. 8, 249-265 (1997).

[51] Moore, G., Seiberg, N.: Lectures on RCFT. In: Physics, Geometry and Topology, H. C. Lee (ed.), Nato ASI Series, Vol. 238, (1990) pp.263-361.

[52] Müger, M.: From subfactors to categories and topology II. The quantum double of tensor categories and subfactors. J. Pure Appl. Alg. 180, 159-219 (2003)

[53] Ostrik, V.: Module categories over the Drinfeld double of a finite group. Inter. Math. Research Notices 27, 1507-1520 (2003); arXiv: math.QA/0202130

[54] Peters, E.: A planar algebra construction of the Haagerup subfactor. arXiv: 0902.1294

[55] Rehren, K.-H.: Braid group statistics and their superselection rules. In: The algebraic theory of superselection sectors. (Palermo 1989), Singapore: World Scientific 1990, pp. 333-355.

[56] Schellekens, A.N., Warner, N.P.: Conformal subalgebras of Kac-Moody algebras. Phys. Rev. D34, 3092-3096 (1986).

[57] Sutherland, C.: Cohomology and extensions of von Neumann algebras. I, II. Publ. RIMS, Kyoto Univ. 16, 135-174 (1980).

[58] Turaev, V.G.: Quantum Invariants of Knots and 3-manifolds. de Gruyter Studies in Mathematics, vol 18. Walter de Gruyter, Berlin, 1994.

[59] Walton, M.A.: Conformal branching rules and modular invariants. Nucl. Phys. B322, 775-790 (1989).

[60] Witten, E.: The search for higher symmetry in string theory. Physics and mathematics of strings. Philos. Trans. Roy. Soc. London Ser. A329 (1989), no. 1605, 349357.

[61] Xu, F.: Mirror extensions of local nets. Commun. Math. Phys. 270, 835-847 (2007).

[62] Zhu, Y.: Modular invariance of characters of vertex operator algebras. J. Amer. Math. Soc. 9, 237-302 (1996). 